\title{
26 NEW MIRA VARIABLES FROM THE ZTF SURVEY
}

\author{
BERNHARD, KLAUS ${ }^{1,3,4}$ AND HÜMMERICH, STEFAN $2,3,4$ \\ 1)_A-4030 Linz, Austria; e-mail: klaus . bernhard@liwest .at \\ 2) D-56338 Braubach, Germany; e-mail: ernham@rz-online .de \\ 3) Bundesdeutsche Arbeitsgemeinschaft für Veränderliche Sterne e.V. (BAV), Munsterdamm 90, D-12169 Berlin \\ 4) American Association of Variable Star Observers (AAVSO), 49 Bay State Road, Cambridge, MA 02138, USA
}

\begin{abstract}
We have continued our search for hitherto unregistered Mira variables in publicly available sky survey data, using observations from the Zwicky Transient Facility and the information provided in the Zwicky Transient Facility Suspected Variables Catalog, which led to the discovery of 26 new Mira stars. Essential data as well as light curves and phase plots for all stars are presented.
\end{abstract}

\section{Introduction}

Basically all stars on the red and asymptotic giant branches brighter than a certain magnitude limit are intrinsically variable. These stars constitute the class of long-period variables, which, because of their intrinsic brightness, are well suited as distance indicators and allow the study of the stellar populations of the Milky Way. The Mira stars are among the most extreme of these variables. According to the definition in the General Catalog of Variable Stars (GCVS; Samus et al. 2017), they show well-defined periodicity with periods in the range of 80 to 1000 days and light amplitudes that, per definitionem, exceed 2.5 magnitudes in $V$. This amplitude cut-off, however, is rather arbitrarily chosen, and there seems to be no real physical distinction but rather a smooth division between Mira stars and other long-period variables of similar properties.

We here report new results from our search for hitherto unregistered Mira variables, which was previously conducted using data from the MACHO survey (Bernhard, 2011; Huemmerich and Bernhard, 2012; Bernhard and Huemmerich, 2013).

\section{Observations and analysis}

The Zwicky Transient Facility (ZTF) is a sky survey that had first light at Palomar Observatory in 2017 and is monitoring the sky down to a limiting magnitude of $r \sim 20.6$ mag. To this end, a camera with a 47 square degree field of view mounted on an 48 -inch Schmidt telescope is employed. The ZTF observes the entire northern and large parts of the southern 
sky and produces high-quality photometric data in three bands $(g, r$, and $i)$, which are made available to the public in consecutive data releases (Bellm et al. 2019a; Bellm et al. 2019b; Masci et al. 2019). The here presented analysis is based on data from the fourth data release (DR $)^{1}$, which contains 7,683,572 observations in the $g$ band, 10,345,520 in the $r$ band, and 465,380 observations in the $i$ band.

To discover new Mira variables, the light curves of objects without a good classification from the ZTF Suspected Variables Catalog (Table 6, 1 million objects) by Chen et al. (2020) were downloaded and examined in detail. No preprocessing of the light curves was performed.

Since the cool Mira stars are considerably brighter in $r$ than in $g$, the quality of the $r$ band light curves is generally much superior for these object. Unfortunately, no $i$ band data were available for our candidate stars, which is not surprising, as the number of measurements in this band is still rather small ( $~ 5 \%$ of observations in DR4). Therefore, we visually inspected all objects from the catalog with a variability amplitude of $\operatorname{amp}(r)>3$ mag and a colour index of 2MASS $\left(J-K_{s}\right)>1.0 \mathrm{mag}$. Promising candidates were checked against the AAVSO International Variable Star Index (VSX; Watson et al., 2006) to find new Mira stars. In this way, several objects designated as type VAR, MISC or L (long-period variables with irregular light curves) in the VSX could be identified as Mira variables. The ZTF data sets were dowloaded from the IRSA Website using PERANSO (Paunzen \& Vanmunster 2016) and analysed with the ANOVA method, as implemented in this program package. The typical ZTF data set used in this study consists of measurements with a time baseline of about 900 days. Therefore, the longest detectable period is about 450 days; any variables with longer periods will have been missed by our approach.

\footnotetext{
${ }^{1}$ https://www.ztf.caltech.edu/page/dr4
} 


\section{Results}

In the above described manner, 26 new Mira variables were identified. Essential data for these objects are presented in Table 1. Figure 2 (Appendix) presents the phase plots and JD light curves of all new Mira variables. Figure 3 (Appendix) illustrates the corresponding sky regions.

Table 1: Essential data for the Mira variables identified in this study. The columns denote: (1) ZTF ID; (2) RA, DEC (J2000); (3) variability type listed in the VSX; (4) period (d), as derived in this study; (5) epoch of maximum in the $r$ band (JD); (6) minimum magnitude $(r)$; (7) maximum magnitude $(r)$; (8) colour index 2MASS $\left(J-K_{s}\right)$ (mag).

\begin{tabular}{|c|c|c|c|c|c|c|c|}
\hline ID & 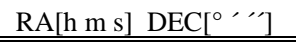 & VSX & period [d] & epoch [JD] & $\min [\mathrm{mag}]$ & $\max [\mathrm{mag}]$ & $\mathrm{J}-\mathrm{K}$ [mag] \\
\hline ZTFJ070812.40-194557.1 & $070812.40-194557.1$ & MISC & 414 & 2458391 & $<15.8$ & 12.5 & 1.855 \\
\hline ZTFJ070912.44-191159.4 & $070912.44-191159.4$ & & 271 & 2458430 & 18.7 & 14.2 & 1.597 \\
\hline ZTFJ173737.82-121047.1 & $173737.82-121047.1$ & $\mathrm{~L}:$ & 240 & 2458670 & 16.7 & 12.7 & 1.548 \\
\hline ZTFJ174046.66-055611.9 & $174046.66-055611.9$ & & 197 & 2458617 & $<16.3$ & 12.3 & 1.600 \\
\hline ZTFJ174109.82-015331.2 & $174109.82-015331.2$ & & 234 & 2458618 & 17.2 & 13.3 & 1.592 \\
\hline ZTFJ174908.60-133931.5 & $174908.60-133931.5$ & MISC & 247 & 2458997 & 17.1 & 12.7 & 1.567 \\
\hline ZTFJ174935.11-142533.9 & $174935.11-142533.9$ & VAR & 203 & 2458608 & 16.5 & 12.5 & 1.462 \\
\hline ZTFJ175156.13-112355.2 & $175156.13-112355.2$ & & 218 & 2458606 & 17.2 & 13.5 & 1.632 \\
\hline ZTFJ175202.89-120213.0 & $175202.89-120213.0$ & & 266 & 2458257 & 18.2 & 13.5 & 1.491 \\
\hline ZTFJ175416.17-090917.0 & $175416.17-090917.0$ & $\mathrm{~L}:$ & 263 & 2458653 & 17.6 & 14.7 & 1.686 \\
\hline ZTFJ175457.68-123542.7 & $175457.68-123542.7$ & & 243 & 2458329 & 18.0 & 13.6 & 1.679 \\
\hline ZTFJ175527.30-103939.8 & $175527.30-103939.8$ & $\mathrm{~L}:$ & 232 & 2458541 & 17.8 & 13.6 & 1.695 \\
\hline ZTFJ175924.69-052150.2 & $175924.69-052150.2$ & & 251 & 2458217 & 19.9 & 15.6 & 1.910 \\
\hline ZTFJ180312.70-144808.1 & $180312.70-144808.1$ & & 226 & 2459020 & 19.7 & 15.5 & 1.887 \\
\hline ZTFJ180329.13-040204.5 & $180329.13-040204.5$ & & 278 & 2458334 & 19.7 & 14.9 & 2.065 \\
\hline ZTFJ180341.17+003614.0 & $180341.17+003614.0$ & L: & 276 & 2458337 & 17.5 & 12.9 & 1.553 \\
\hline ZTFJ180341.59-031849.0 & $180341.59-031849.0$ & & 260 & 2458262 & 19.4 & 15.5 & 2.017 \\
\hline ZTFJ180638.91-163509.8 & $180638.91-163509.8$ & & 241 & 2458640 & 19.0 & 14.7 & 1.897 \\
\hline ZTFJ180640.24-135159.8 & $180640.24-135159.8$ & & 532 & 2458608 & 19.7 & 15.3 & 2.410 \\
\hline ZTFJ180646.55-013545.0 & $180646.55-013545.0$ & & 238 & 2458744 & 19.7 & 15.7 & 1.698 \\
\hline ZTFJ180729.85-013438.1 & $180729.85-013438.1$ & & 246 & 2458680 & 18.9 & 15.0 & 1.968 \\
\hline ZTFJ180826.00-174129.0 & $180826.00-174129.0$ & & 232 & 2458681 & 18.7 & 14.6 & 2.228 \\
\hline ZTFJ184503.44+154335.3 & $184503.44+154335.3$ & & 279 & 2459022 & 18.1 & 14.0 & 1.460 \\
\hline ZTFJ190648.43+130203.5 & $190648.43+130203.5$ & & 396 & 2458805 & 19.0 & 15.5 & 2.373 \\
\hline ZTFJ191008.27+131014.4 & $191008.27+131014.4$ & & 271 & 2458218 & 20.2 & 15.9 & 2.120 \\
\hline ZTFJ191406.43+151810.6 & $191406.43+151810.6$ & VAR & 418 & 2458318 & 18.8 & 14.7 & 1.966 \\
\hline
\end{tabular}


A colour-magnitude diagram and a period-magnitude diagram based on 2MASS photometry (Skrutskie et al., 2006), are presented in Figure 1. For comparison, the diagrams contain the 1286 Mira variables found in the $R_{\mathrm{C}}$-band data of the MACHO data archive by Bernhard (2011), Hümmerich \& Bernhard (2012), and Bernhard \& Hümmerich (2013) as well as the sample of 192 mid-infrared bright (MIBR) Mira variables of Bernhard et al. (2016).

The 26 new ZTF Mira variables fit in well with the MACHO samples in both diagrams. It is noteworthy, however, that none of the new Miras is situated on the 'red tail' of (mostly longperiod) stars with 2MASS $\left(H-K_{s}\right) \geq 1$, which extends to $\left(H-K_{s}\right) \sim 1.5$ and is most pronounced for the MIBR objects (Figure 1, upper panel). This can probably be explained by the fact that the ZTF $r$ band covers somewhat shorter wavelengths than the MACHO $R_{\mathrm{C}}$ band. Consequently, the dust-obscured stars with large $\left(H-K_{s}\right)$ values will be quite faint in the ZTF $r$ band and will have been missed. Therefore, the ZTF Miras cluster in a region of the periodmagnitude diagram (Figure 1, lower panel) where the short-period objects are located.

\section{Conclusions}

In this work, which is part of our framework to search for hitherto unregistered Mira variables, we present 26 new Mira stars with periods in the range of 203 to 418 days, which were identified in the ZTF Suspected Variables Catalog. With recent variable star catalogs containing hundreds of thousands of entries, we expect that it will be possible to extract many more Mira stars from among the unclassified or suspected variable stars in these catalogs. Our future research in this respect will be concerned with the development of refined search criteria to make the identification of Mira stars more efficient.

\section{Acknowledgements:}

This paper made use of data from VizieR catalog access tool, and of the interactive sky atlas Aladin, CDS, Strasbourg, France, the NASA/IPAC Infrared Science Archive and of the International Variable Star Index (VSX) database of the American Association of Variable Star Observers (AAVSO, Cambridge, Massachusetts, USA). 

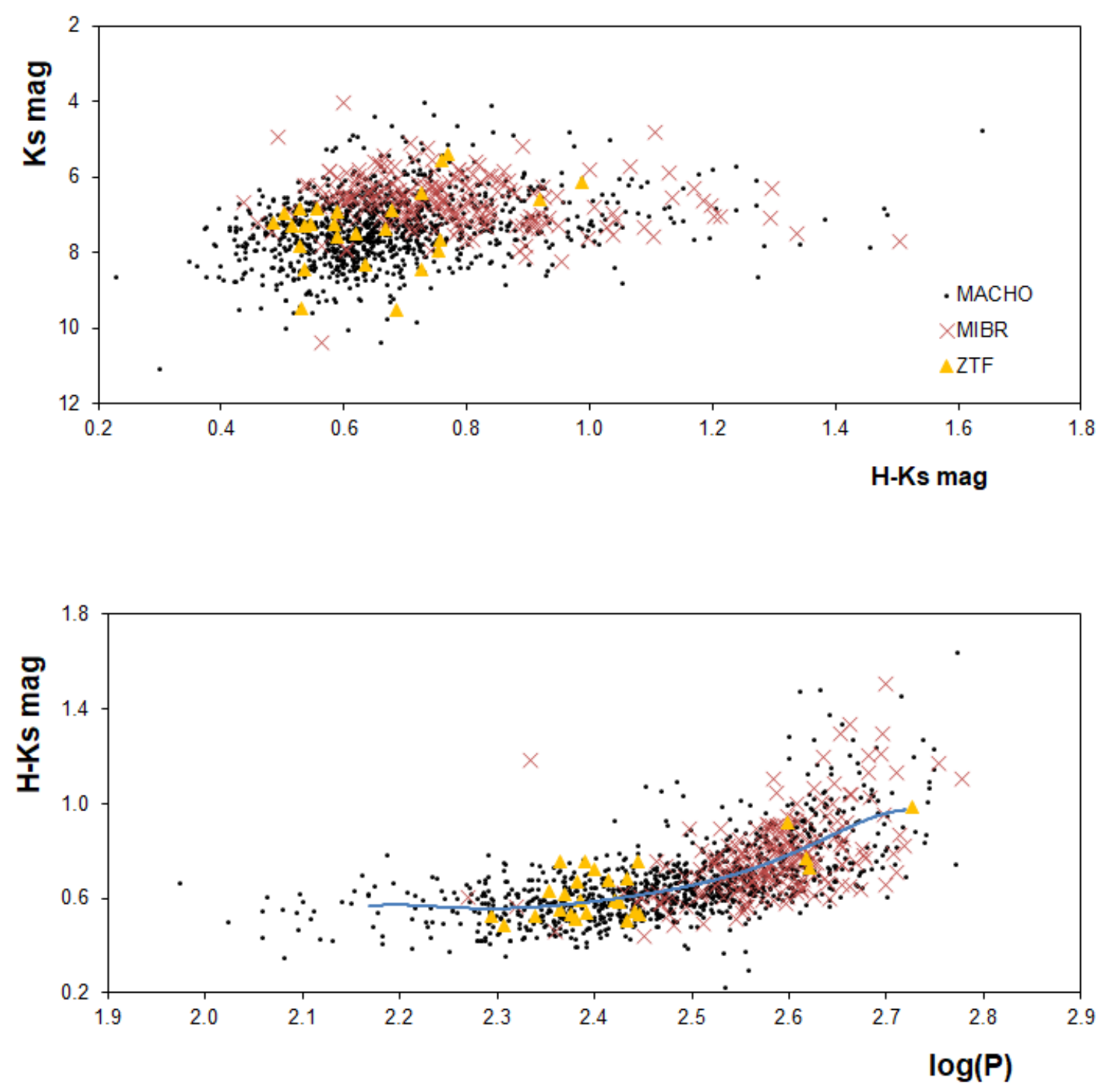

Figure 1 - Colour-magnitude diagram (top) and period-magnitude diagram (bottom) of the MACHO (black dots), MIBR-MACHO (red crosses), and ZTF (yellow triangles) Mira variables. 


\section{References}

Bellm, E. C., Kulkarni, S. R., Graham, M. J., et al., 2019, PASP, 131, 018002 https://ui.adsabs.harvard.edu/abs/2019PASP..131a8002B

Bellm, E. C., Kulkarni, S. R., Barlow, T., et al., 2019, PASP, 131, 068003

https://ui.adsabs.harvard.edu/abs/2019PASP..131f8003B

Bernhard, K., 2011, PZP, 11, 12

http://www.astronet.ru/db/varstars/msg/1249318

Bernhard, K., Hümmerich, S., 2013, OEJV, 159

http://astro.sci.muni.cz/variables/oejv/issues/oejv0159.pdf

Bernhard, K., Uttenthaler, S., Huemmerich, S., 2016, BAVJ, No.10

https://ui.adsabs.harvard.edu/abs/2016BAVJ...10....1B/abstract

Chen, X., Wang, S., Deng, L., de Grijs, R., Yang, M., Tian, H., 2020, ApJS, 249, 18 https://ui.adsabs.harvard.edu/abs/2020ApJS..249...18C

Hümmerich, S., Bernhard, K., 2012, OEJV, 149

http://astro.sci.muni.cz/variables/oejv/issues/oejv0149.pdf

Masci, F. J., Laher, R. R., Rusholme, B., et al., 2019, PASP, 131, 018003

https://ui.adsabs.harvard.edu/abs/2019PASP..131a8003M

Paunzen, E., Vanmunster, T., 2016, AN, 337, 239

https://ui.adsabs.harvard.edu/abs/2016AN....337..239P

Skrutskie, M. F., Cutri, R. M., Stiening, R., et al., 2006, AJ, 131, 1163

https://ui.adsabs.harvard.edu/abs/2006AJ...131.1163S/abstract 


\section{Appendix}

Figure 2 presents the phase plots and JD light curves of all new Mira variables discovered in the present study. Red and green dots denote ZTF $r$ and $g$ band data, respectively. For the construction of the phase plots, the periods given in Table 1 have been used.

Figure 3 illustrates the sky regions of the Mira stars. All plots have been based on DSS2 plates, which were accessed via the ALADIN sky atlas (Bonnarel et al. 2000; Boch \& Fernique 2014). North is at the top, east is at the left.

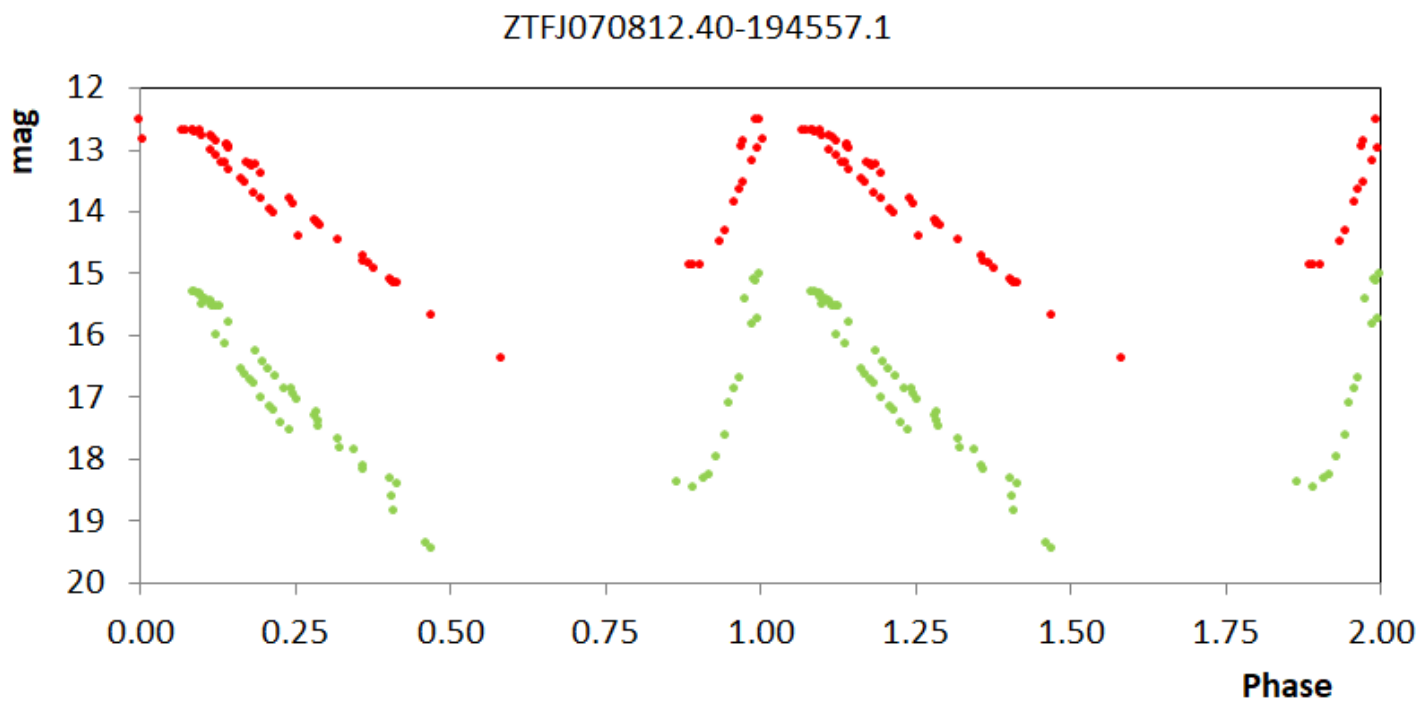

ZTFJ070812.40-194557.1

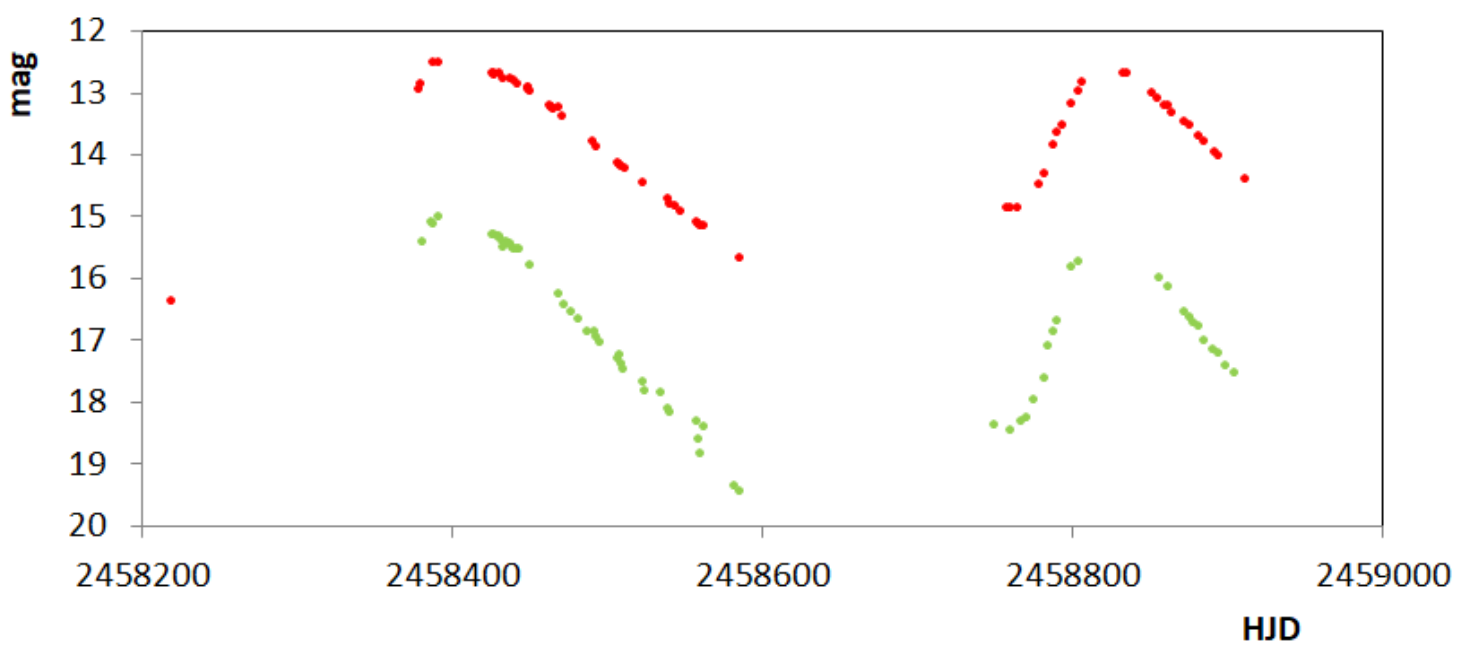

Figure 2.1 - Phase plot and JD light curve of ZTFJ070812.40-194557.1. Red and green dots denote ZTF $r$ and $g$ band data, respectively. The phase plot has been folded with $P=414 \mathrm{~d}$ (Table 1). 
ZTFJ070912.44-191159.4

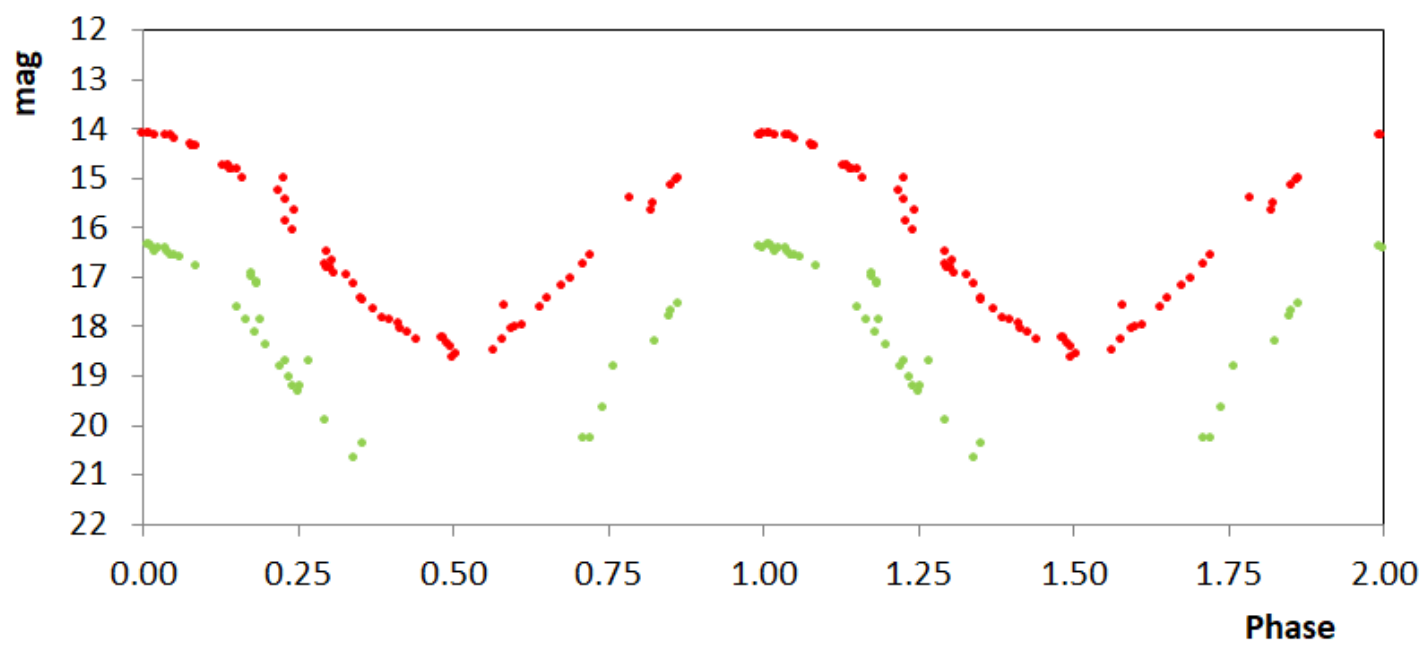

ZTFJ070912.44-191159.4

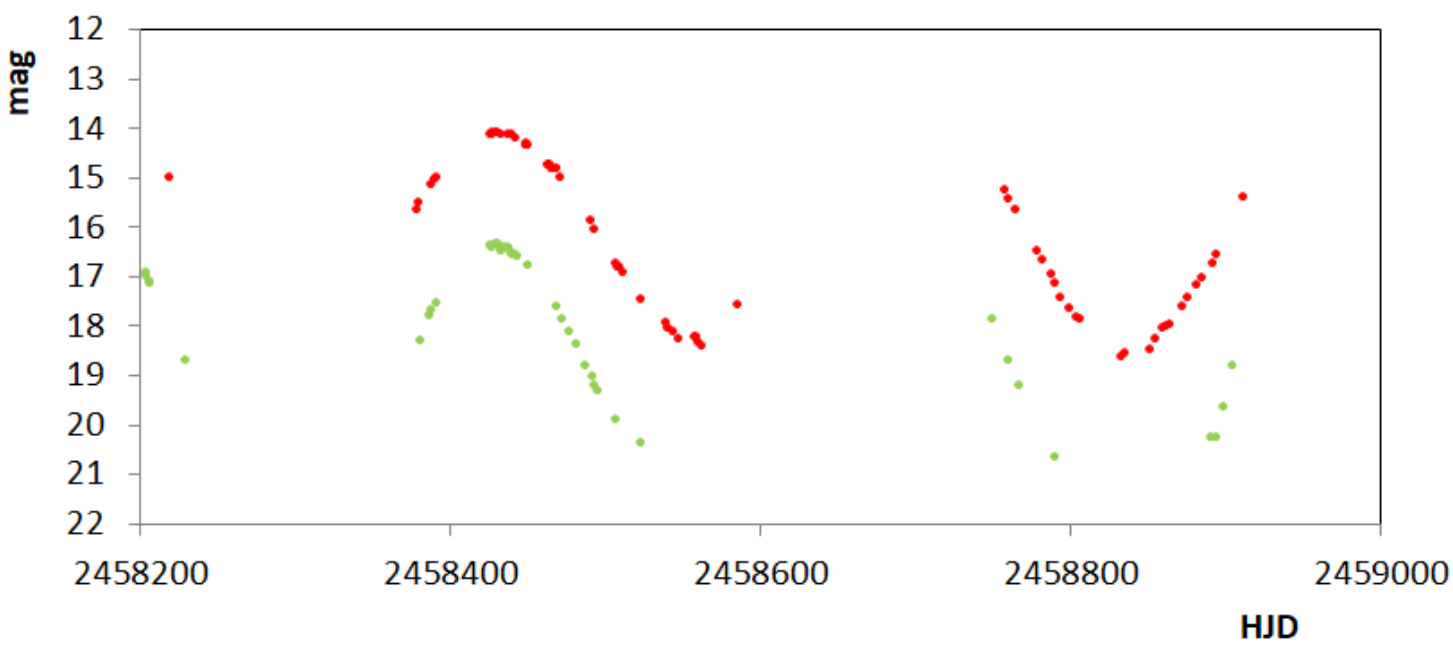

Figure 2.2 - Phase plot and JD light curve of ZTFJ070912.44-191159.4. Red and green dots denote ZTF $r$ and $g$ band data, respectively. The phase plot has been folded with $P=271 \mathrm{~d}$ (Table 1). 
ZTFJ173737.82-121047.1

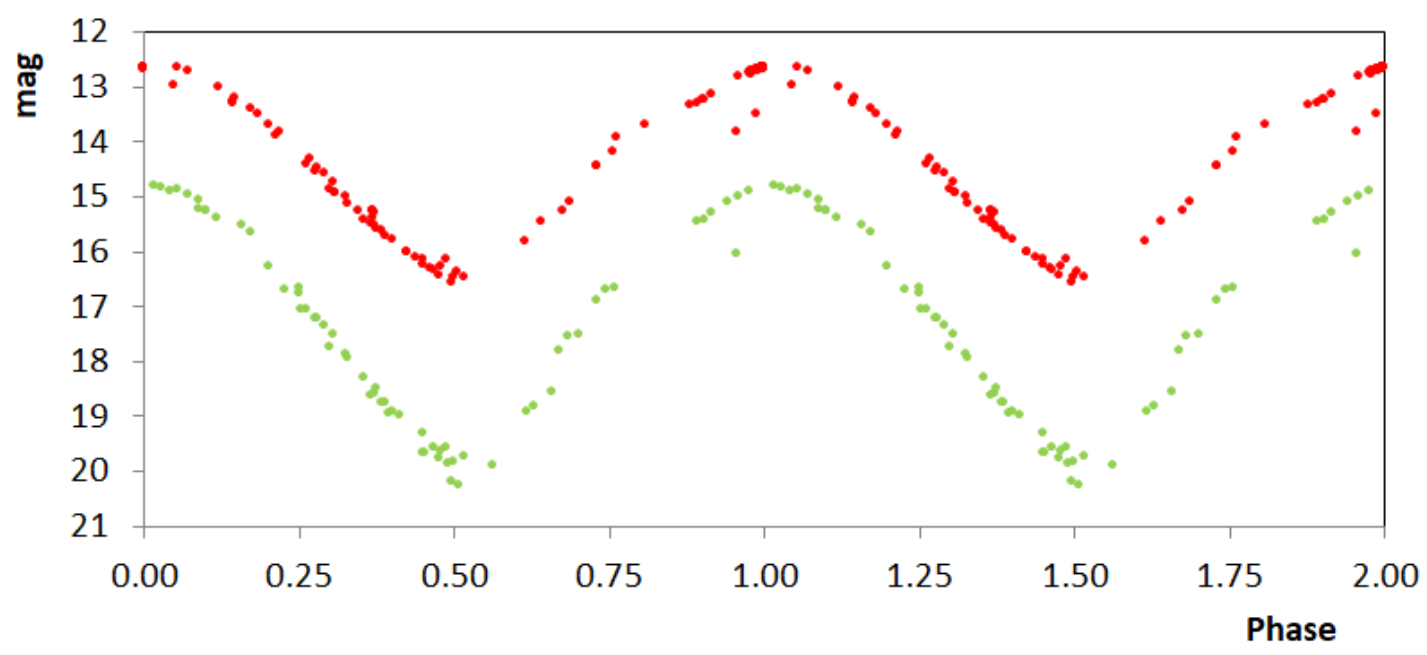

ZTFJ173737.82-121047.1

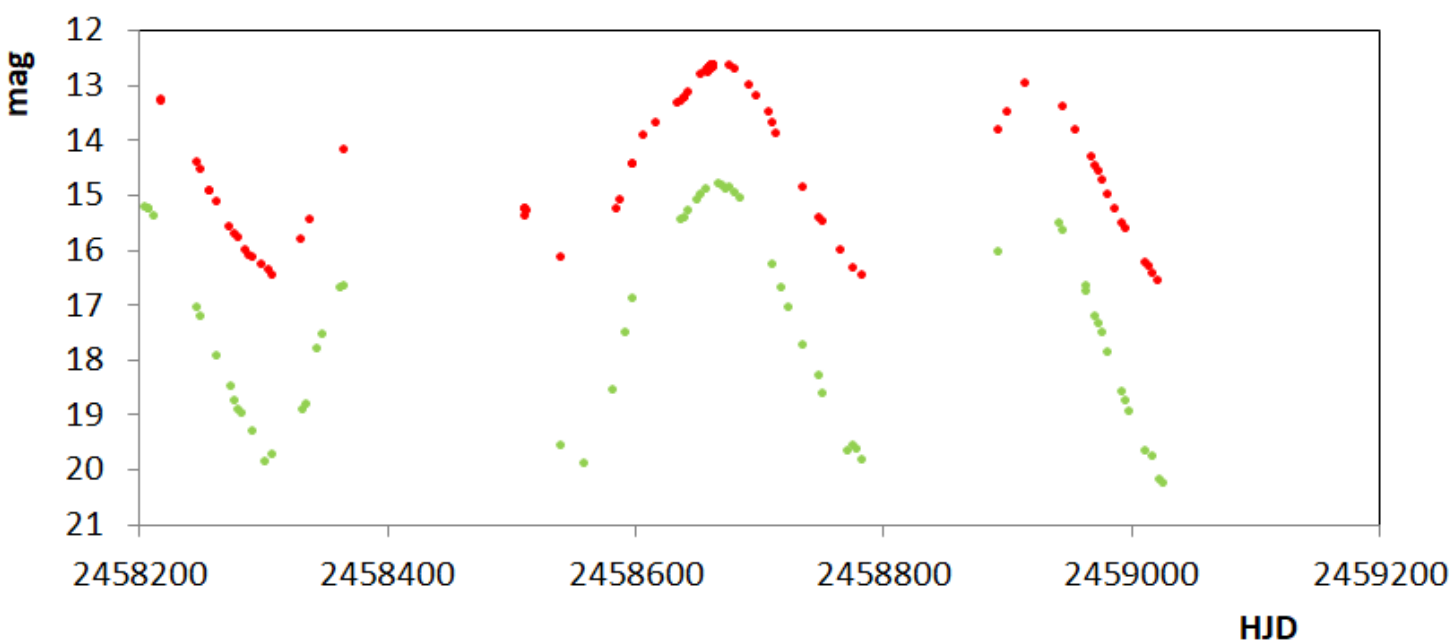

Figure 2.3 - Phase plot and JD light curve of ZTFJ173737.82-121047.1. Red and green dots denote ZTF $r$ and $g$ band data, respectively. The phase plot has been folded with $P=240 \mathrm{~d}$ (Table 1). 


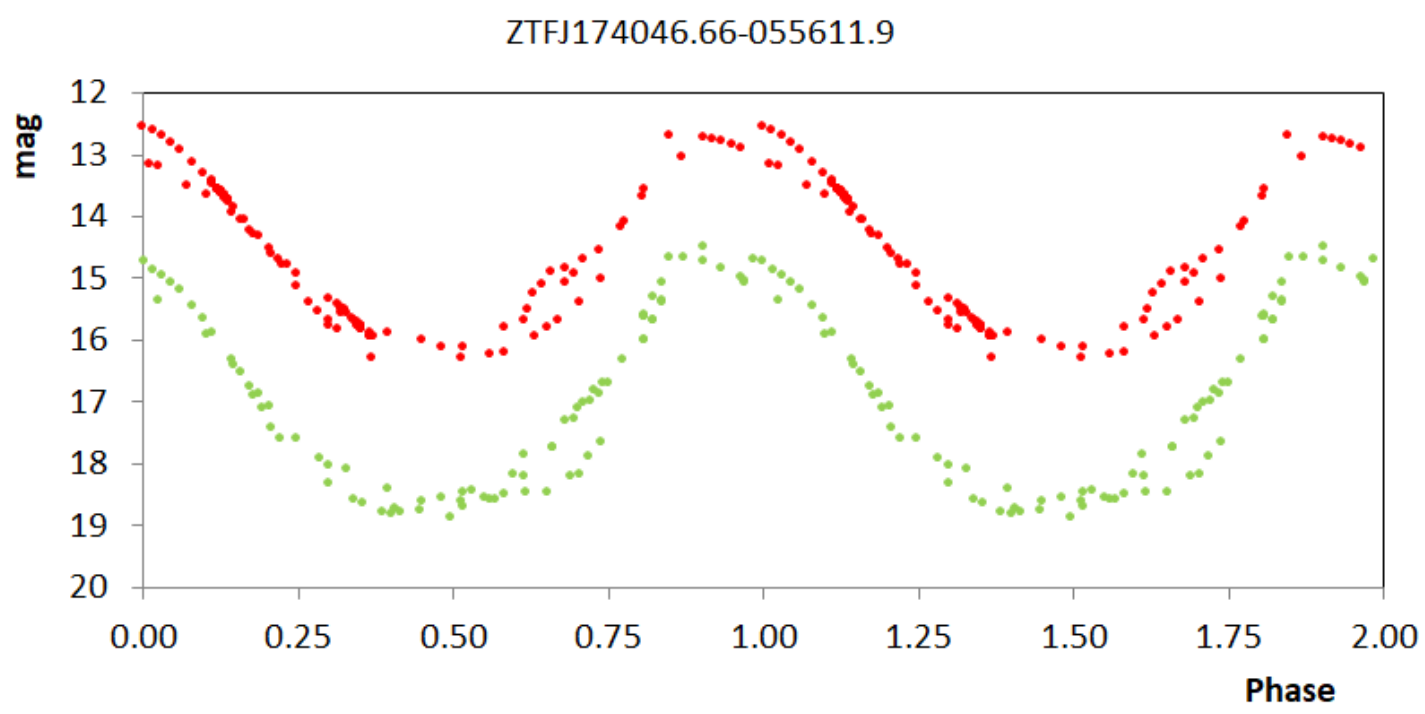

ZTFJ174046.66-055611.9

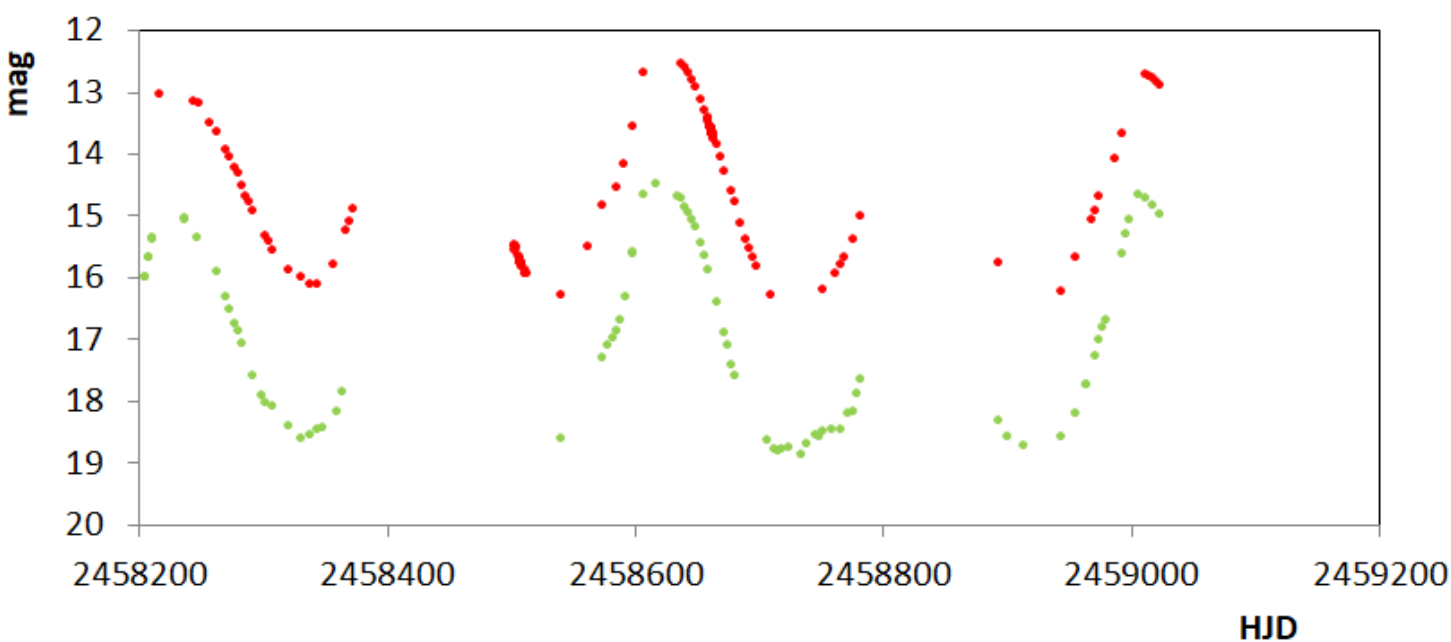

Figure 2.4 - Phase plot and JD light curve of ZTFJ174046.66-055611.9. Red and green dots denote ZTF $r$ and $g$ band data, respectively. The phase plot has been folded with $P=197 \mathrm{~d}$ (Table 1). 
ZTFJ174109.82-015331.2

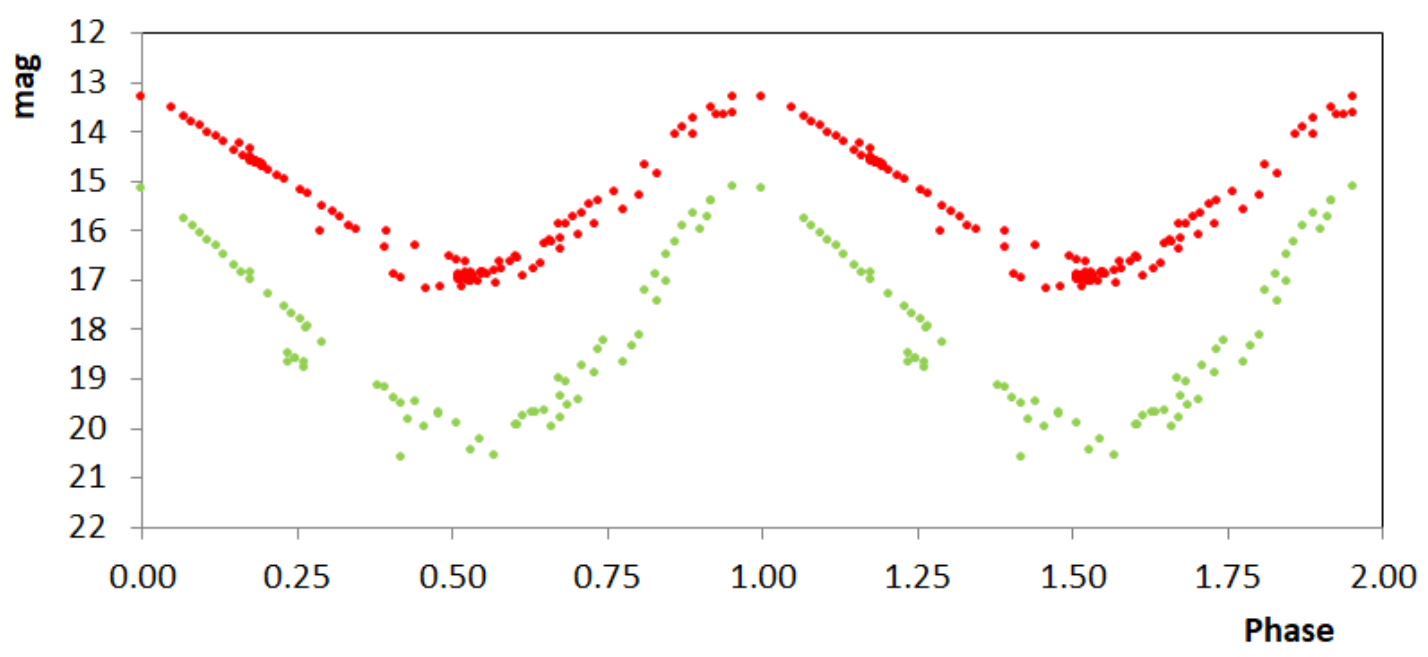

ZTFJ174109.82-015331.2

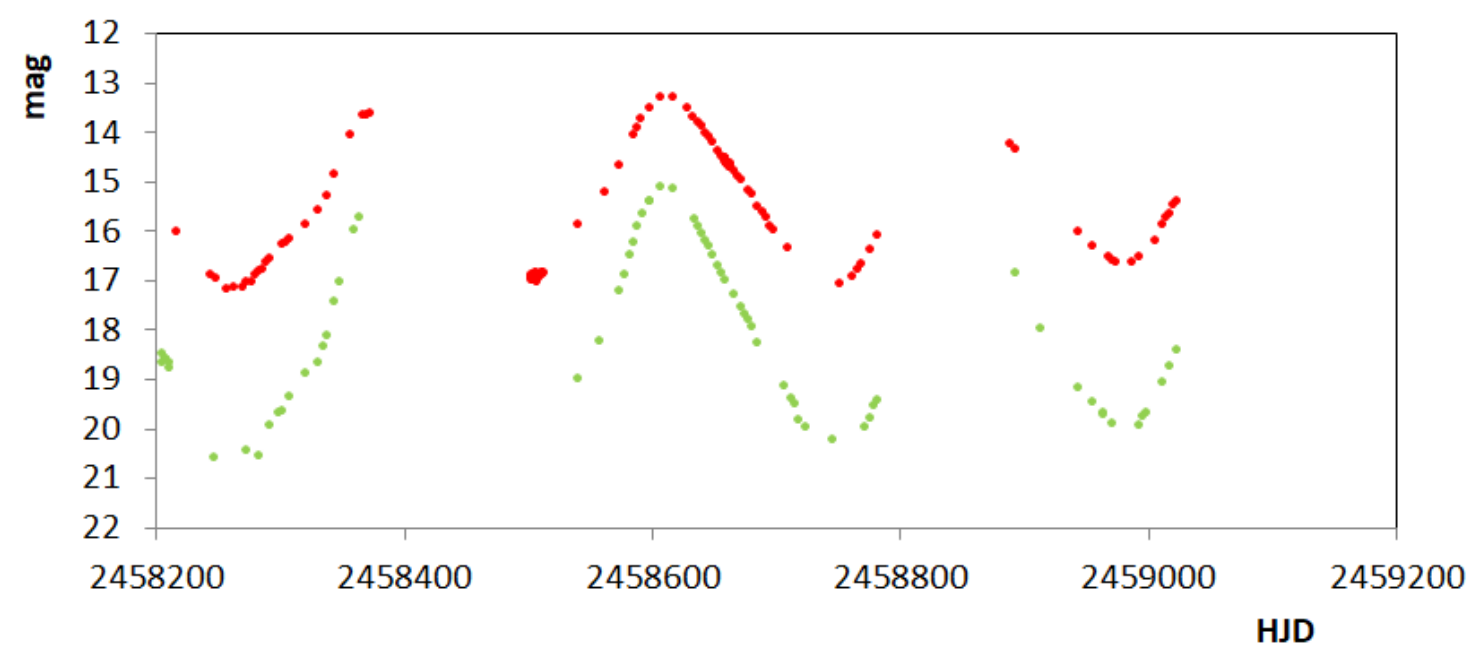

Figure 2.5 - Phase plot and JD light curve of ZTFJ174109.82-015331.2. Red and green dots denote ZTF $r$ and $g$ band data, respectively. The phase plot has been folded with $P=234 \mathrm{~d}$ (Table 1). 
ZTFJ174908.60-133931.5

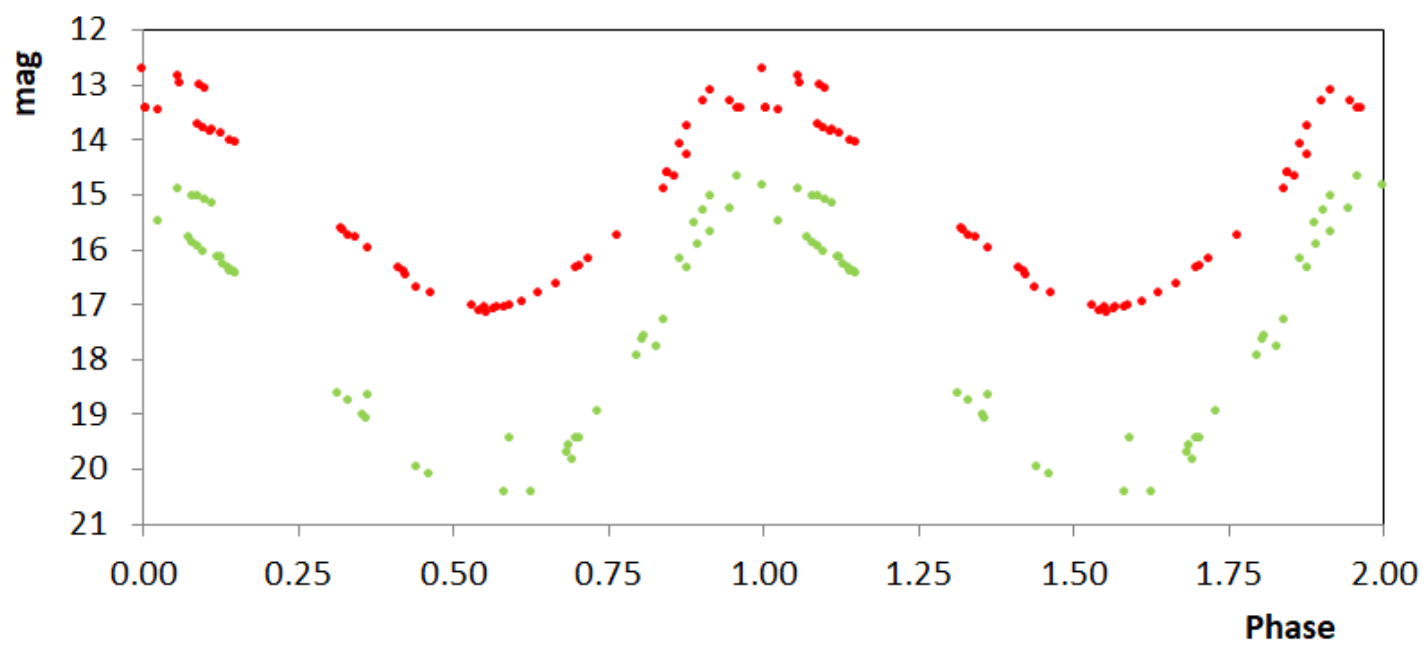

ZTFJ174908.60-133931.5

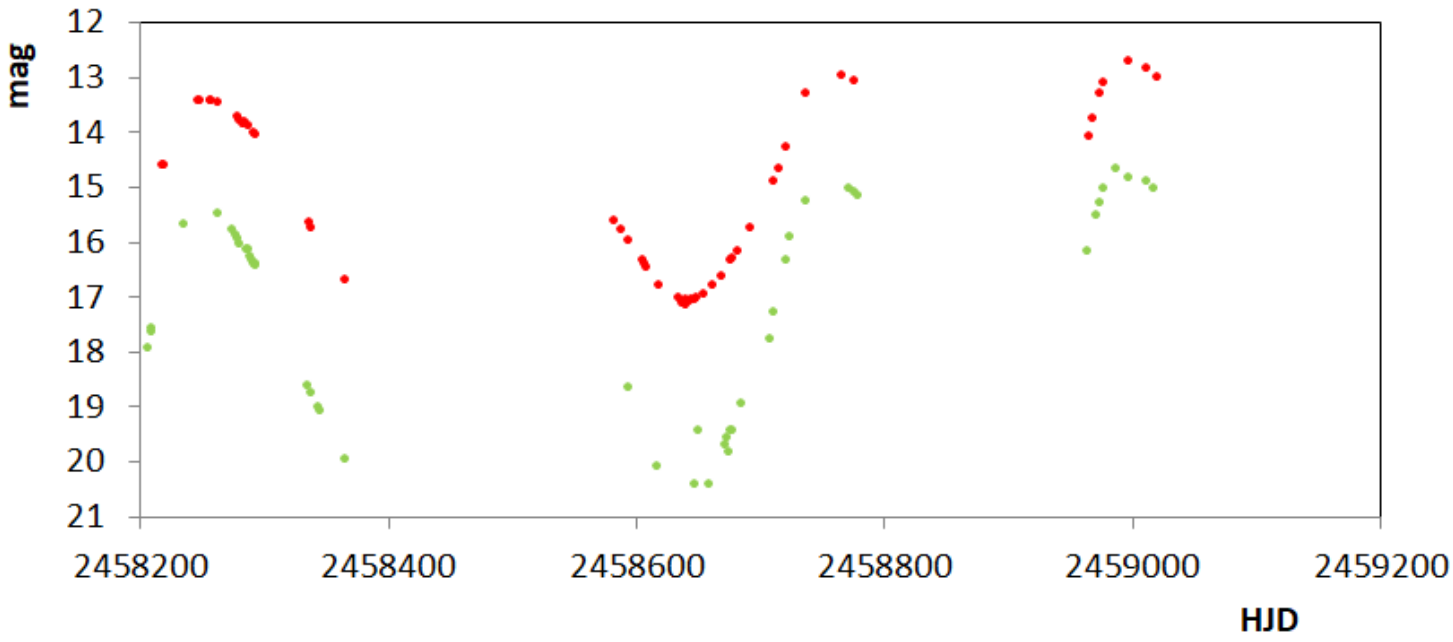

Figure 2.6 - Phase plot and JD light curve of ZTFJ174908.60-133931.5. Red and green dots denote ZTF $r$ and $g$ band data, respectively. The phase plot has been folded with $P=247 \mathrm{~d}$ (Table 1). 
ZTFJ174935.11-142533.9

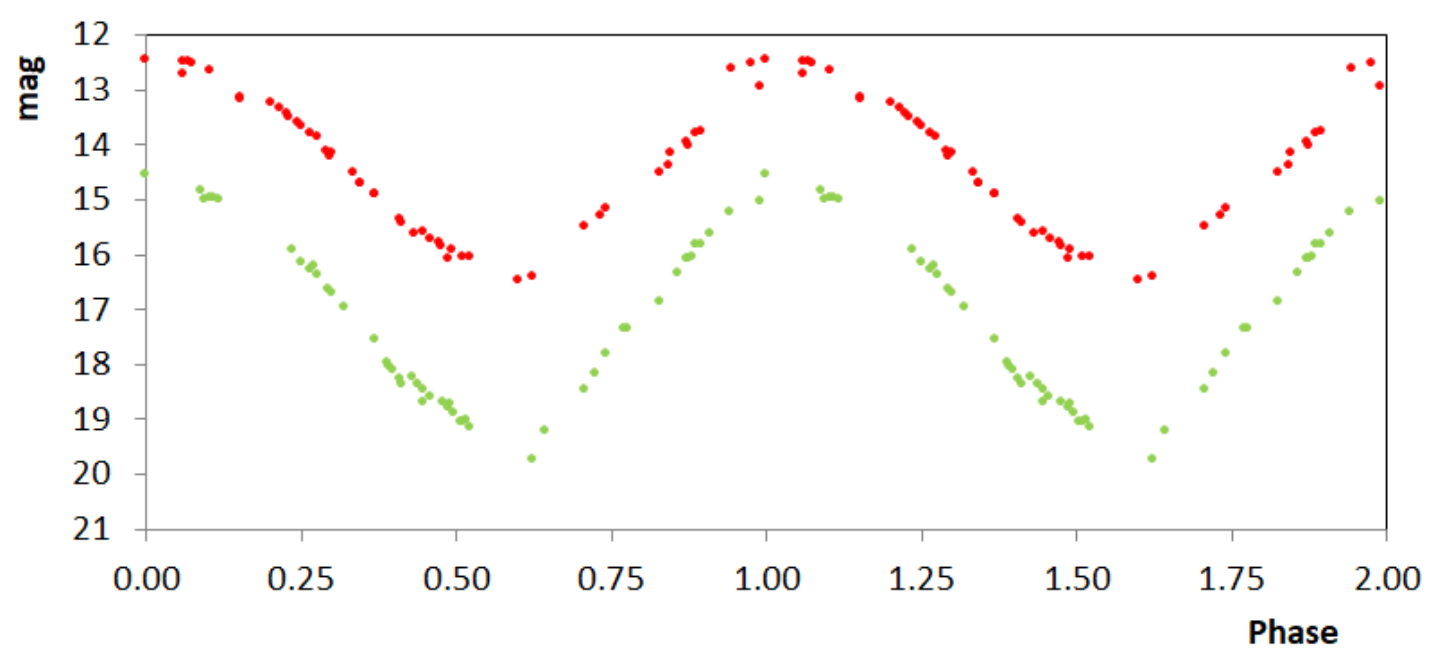

ZTFJ174935.11-142533.9

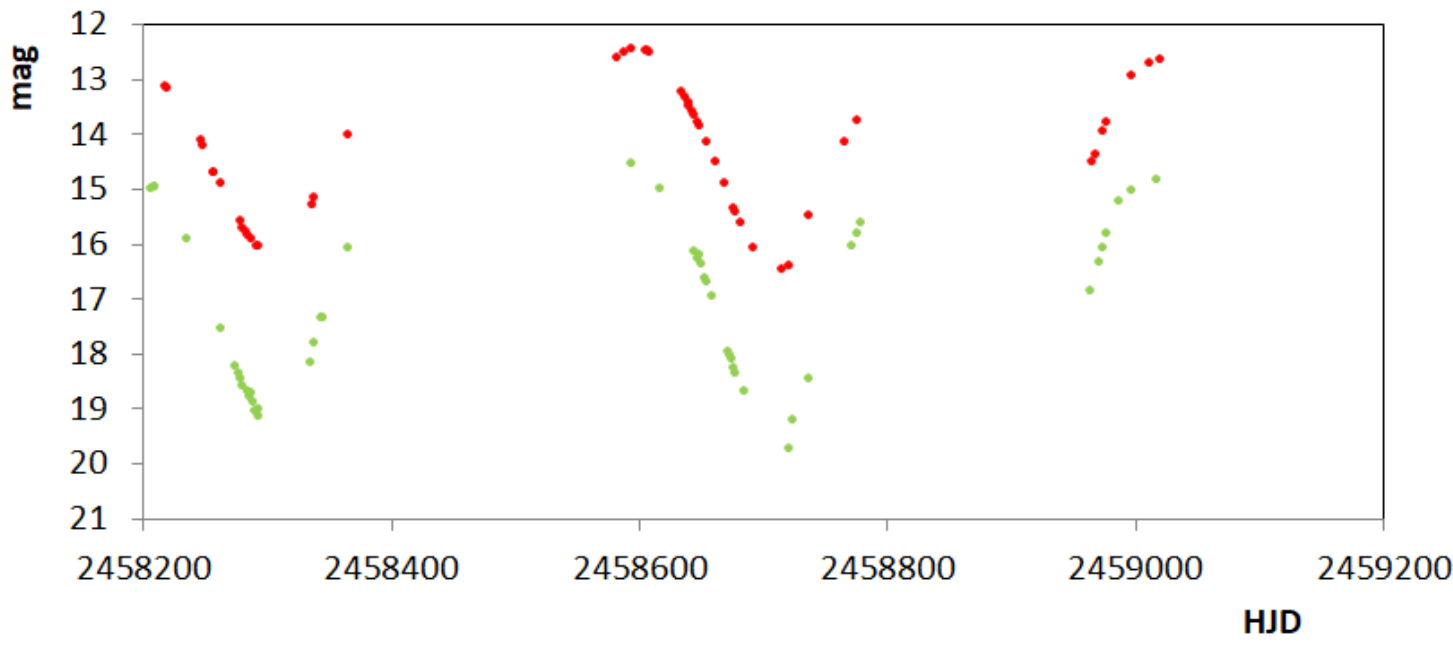

Figure 2.7 - Phase plot and JD light curve of ZTFJ174935.11-142533.9. Red and green dots denote ZTF $r$ and $g$ band data, respectively. The phase plot has been folded with $P=203 \mathrm{~d}$ (Table 1). 
ZTFJ175156.13-112355.2

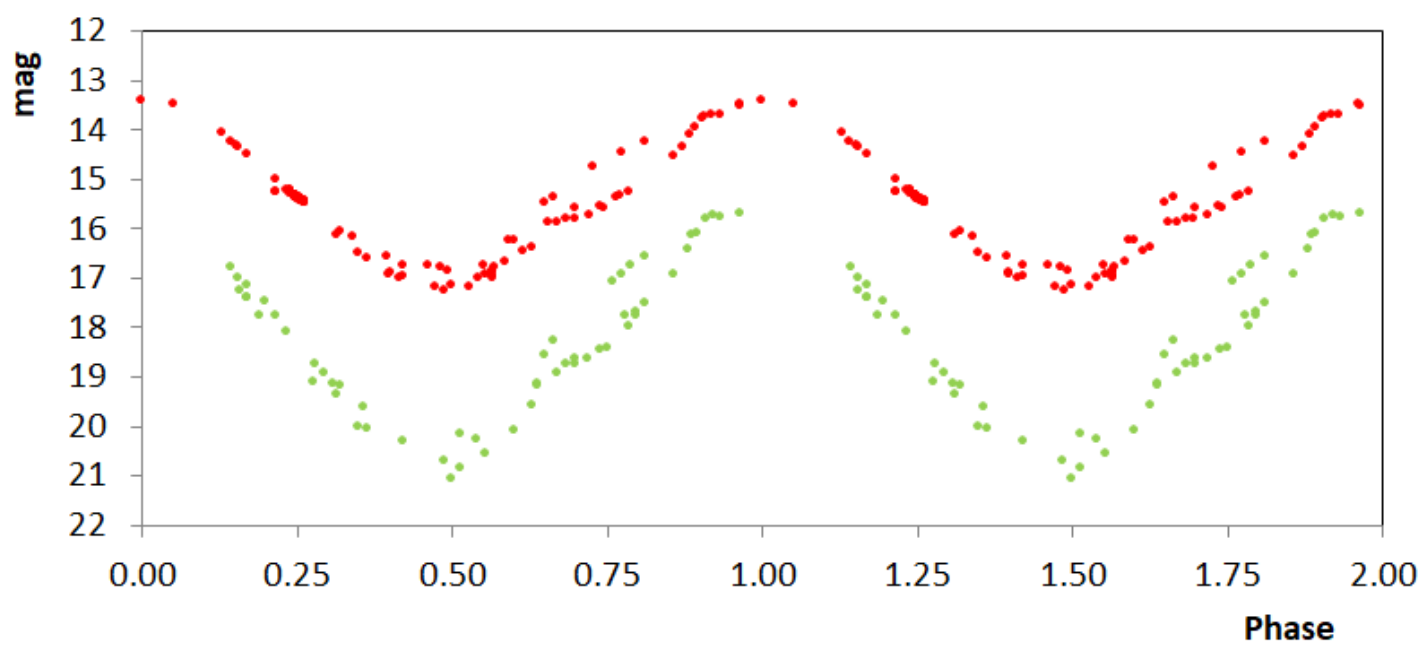

ZTFJ175156.13-112355.2

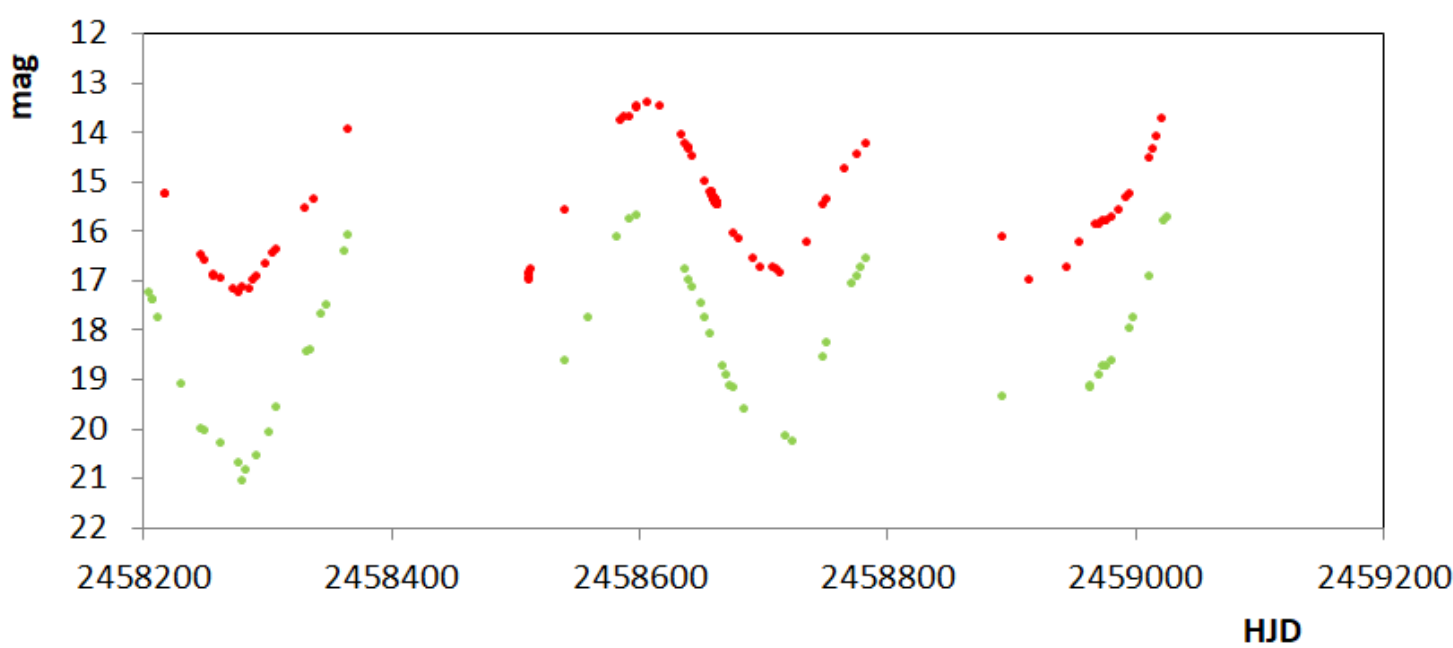

Figure 2.8 - Phase plot and JD light curve of ZTFJ175156.13-112355.2. Red and green dots denote ZTF $r$ and $g$ band data, respectively. The phase plot has been folded with $P=218 \mathrm{~d}$ (Table 1). 
ZTFJ175202.89-120213.0

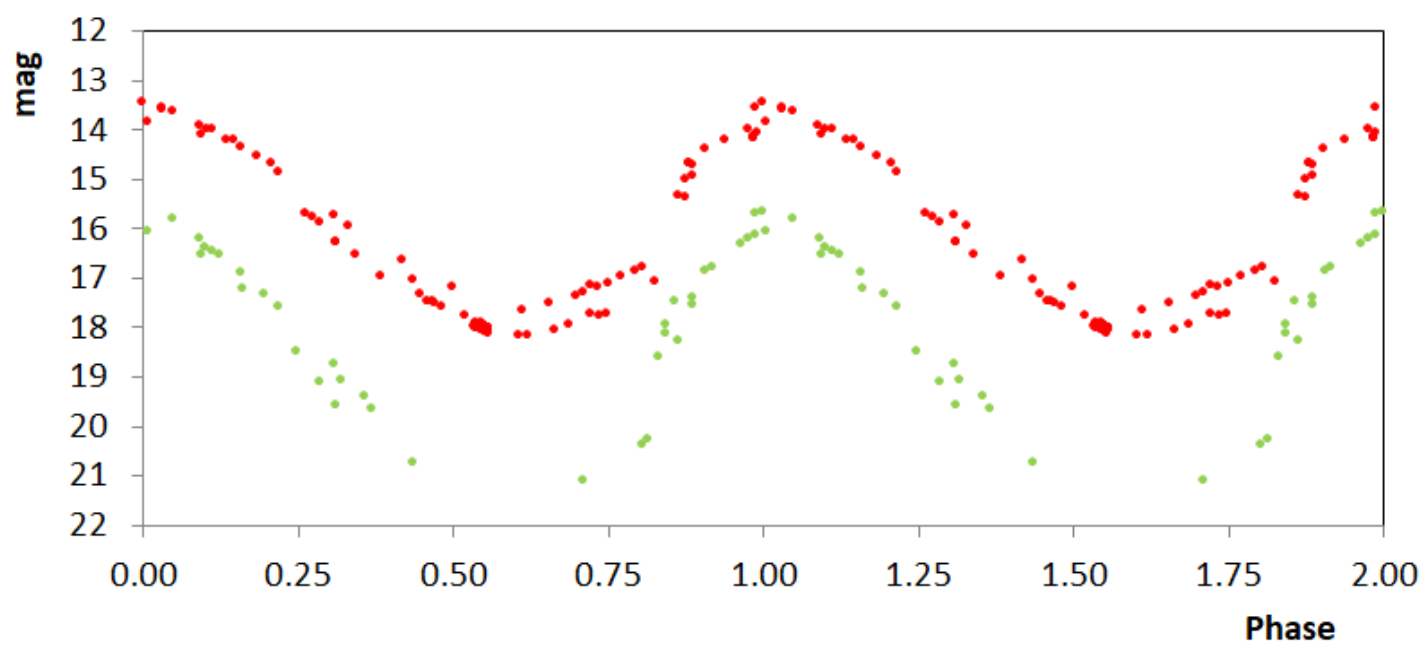

ZTFJ175202.89-120213.0

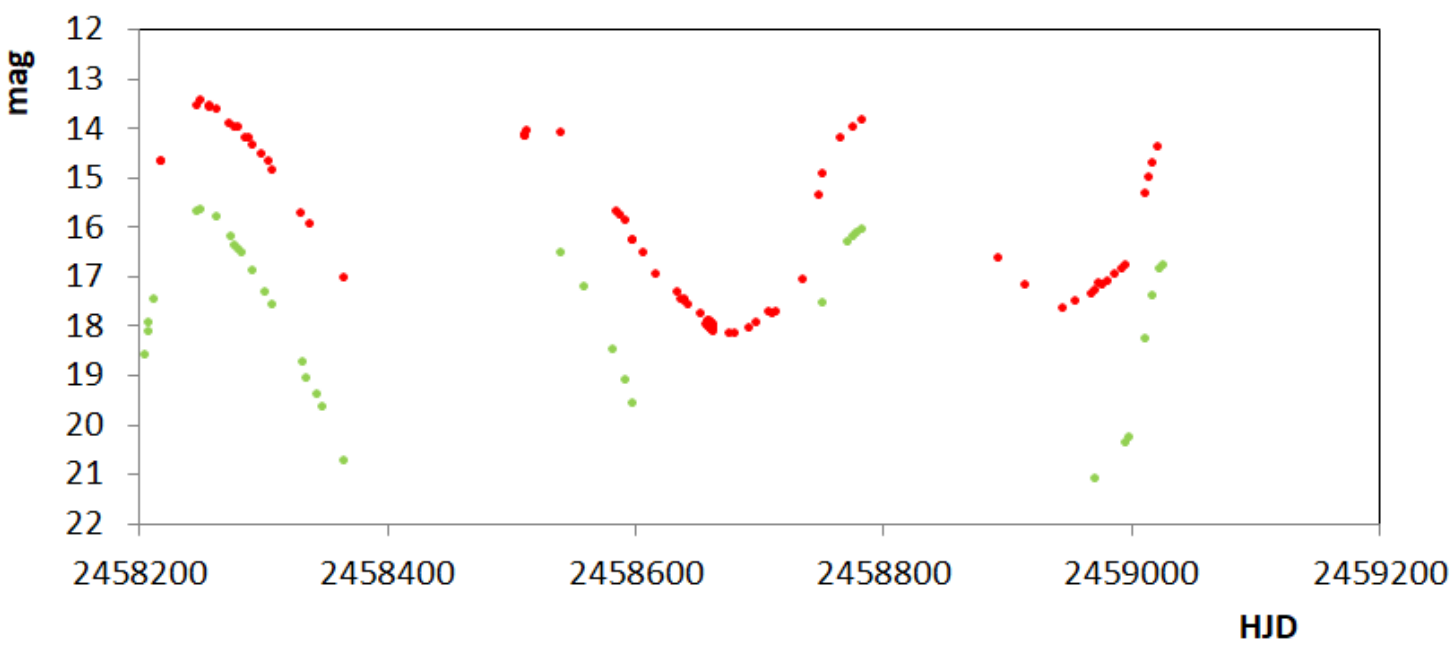

Figure 2.9 - Phase plot and JD light curve of ZTFJ175202.89-120213.0. Red and green dots denote ZTF $r$ and $g$ band data, respectively. The phase plot has been folded with $P=266 \mathrm{~d}$ (Table 1). 


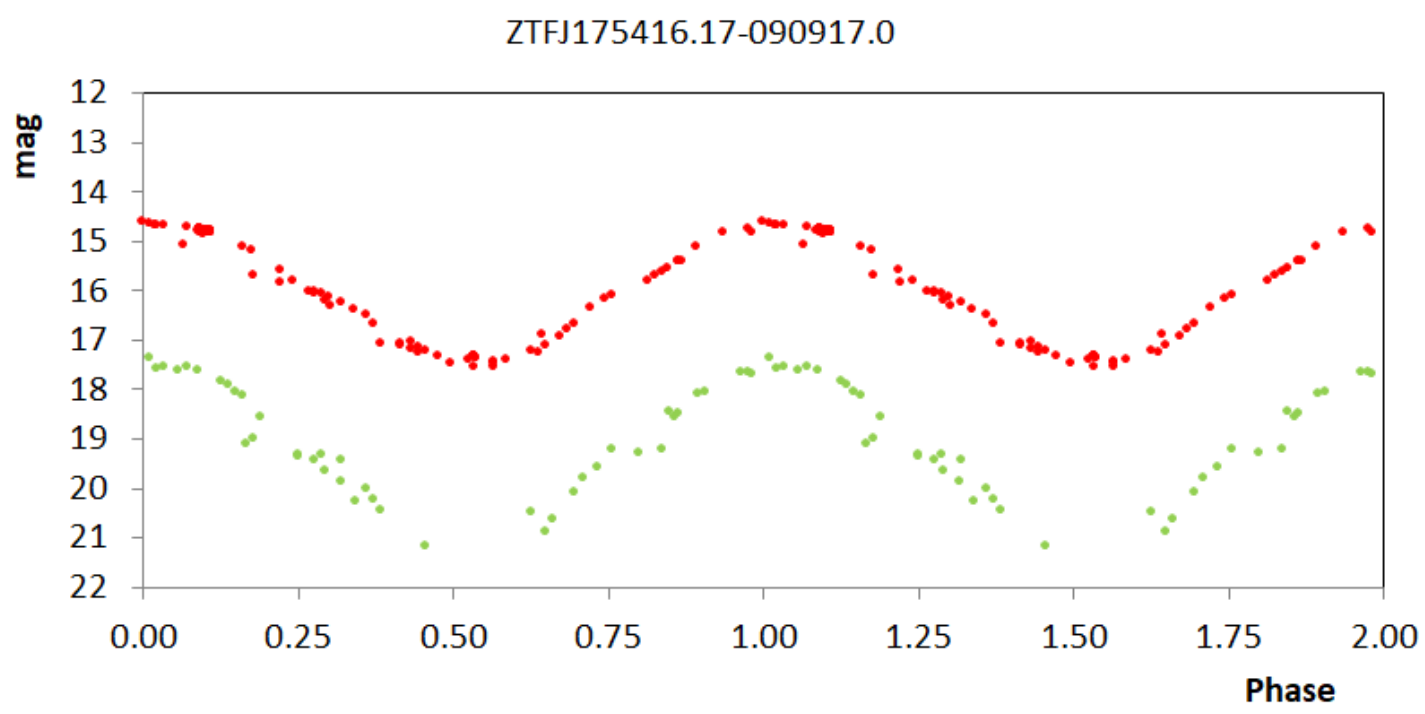

ZTFJ175416.17-090917.0

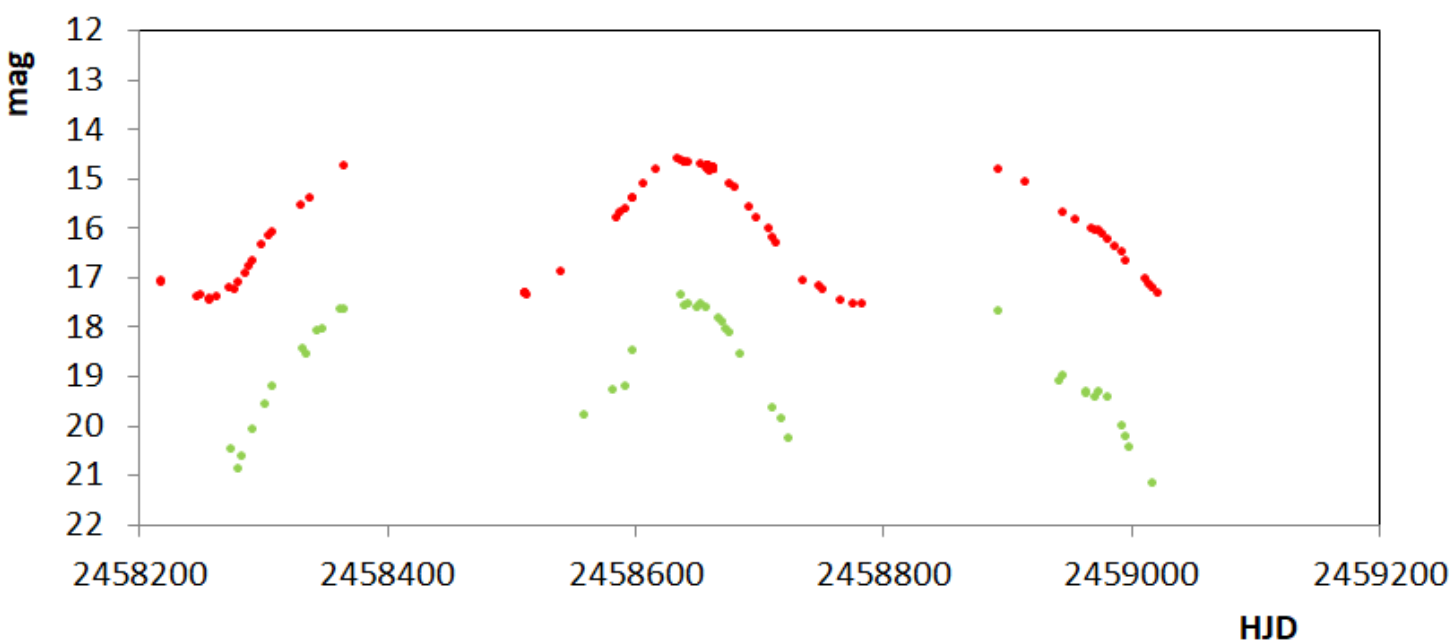

Figure 2.10 - Phase plot and JD light curve of ZTFJ175416.17-090917.0. Red and green dots denote ZTF $r$ and $g$ band data, respectively. The phase plot has been folded with $P=263 \mathrm{~d}$ (Table 1). 
ZTFJ175457.68-123542.7

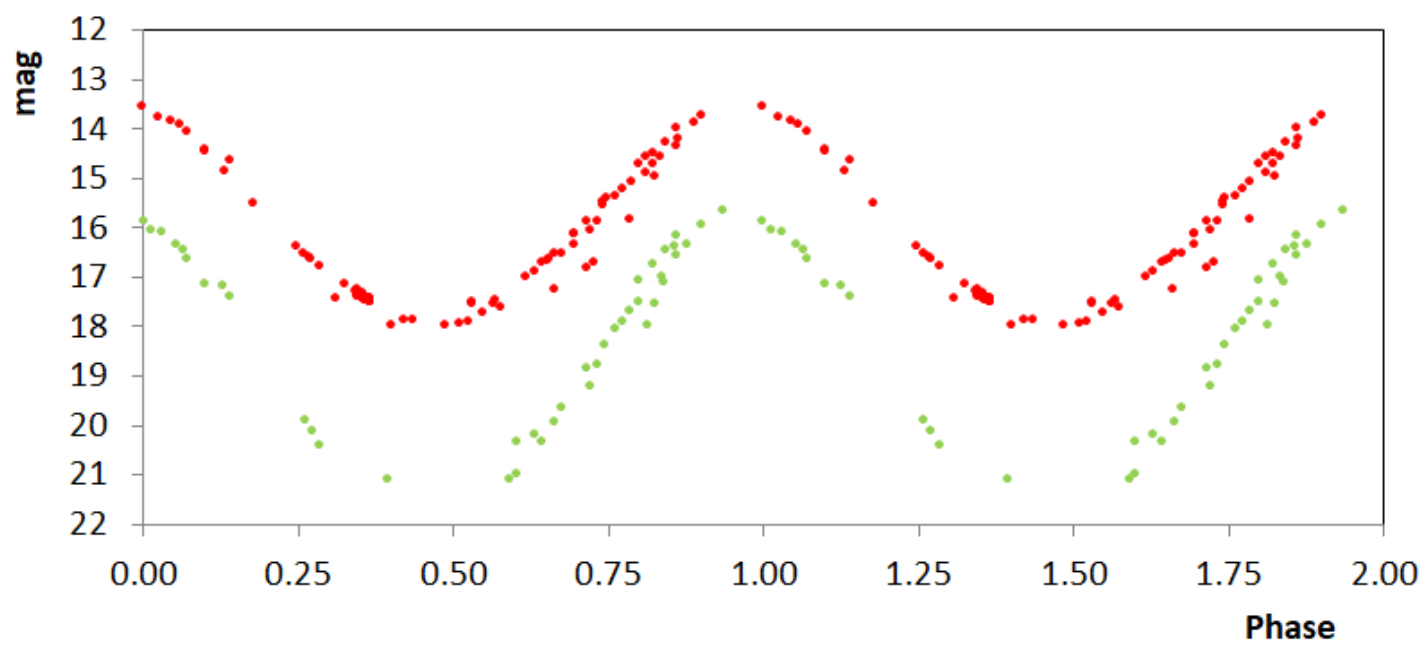

ZTFJ175457.68-123542.7

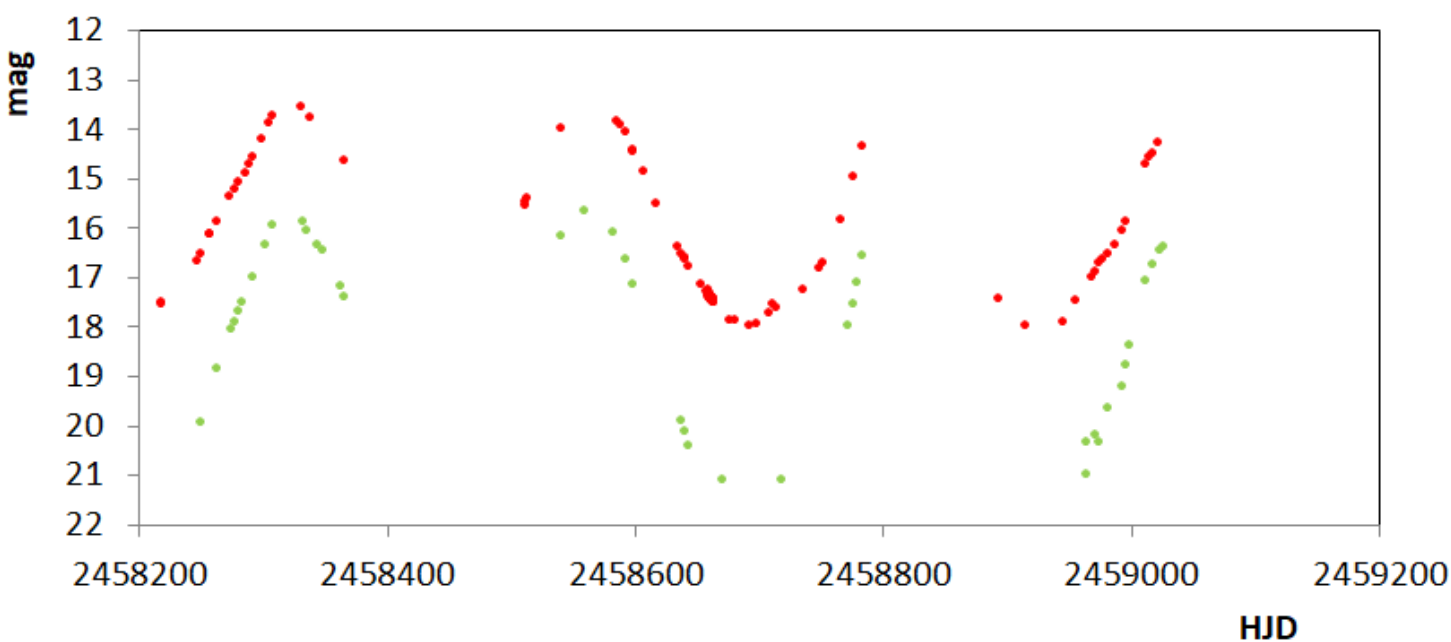

Figure 2.11 - Phase plot and JD light curve of ZTFJ175457.68-123542.7. Red and green dots denote ZTF $r$ and $g$ band data, respectively. The phase plot has been folded with $P=243 \mathrm{~d}$ (Table 1). 
ZTFJ175527.30-103939.8

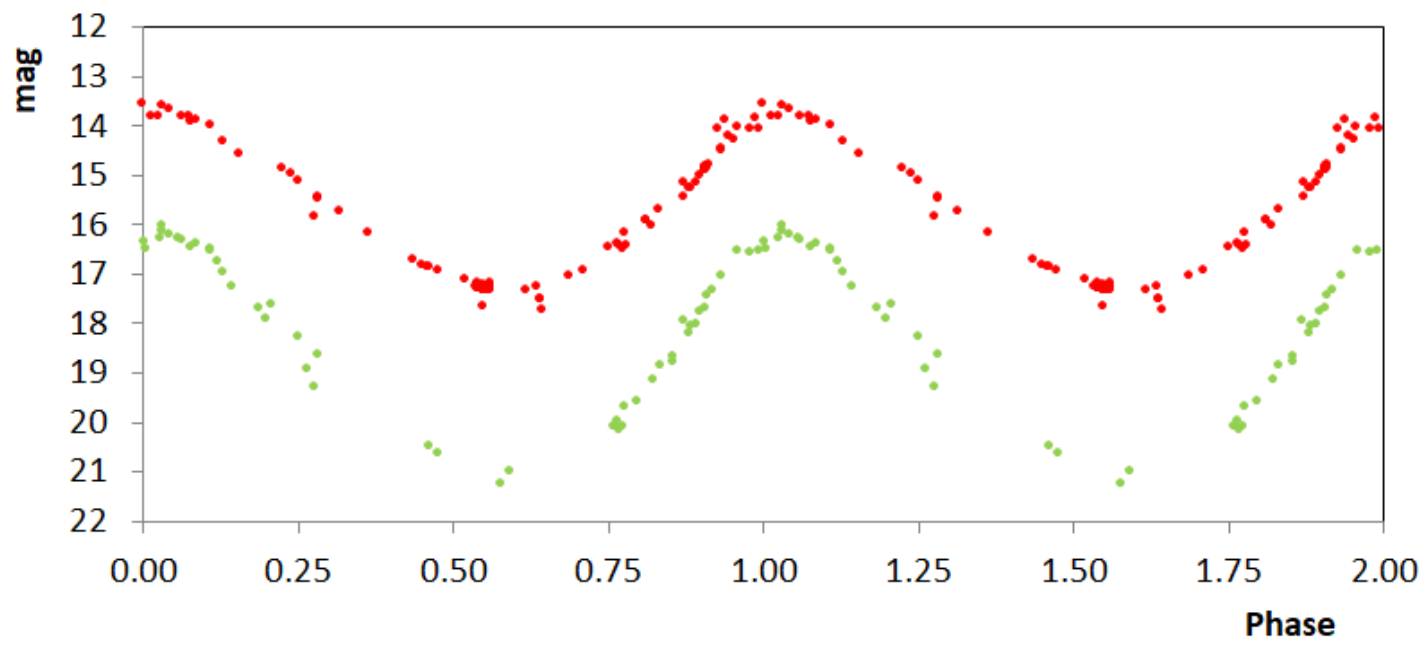

ZTFJ175527.30-103939.8

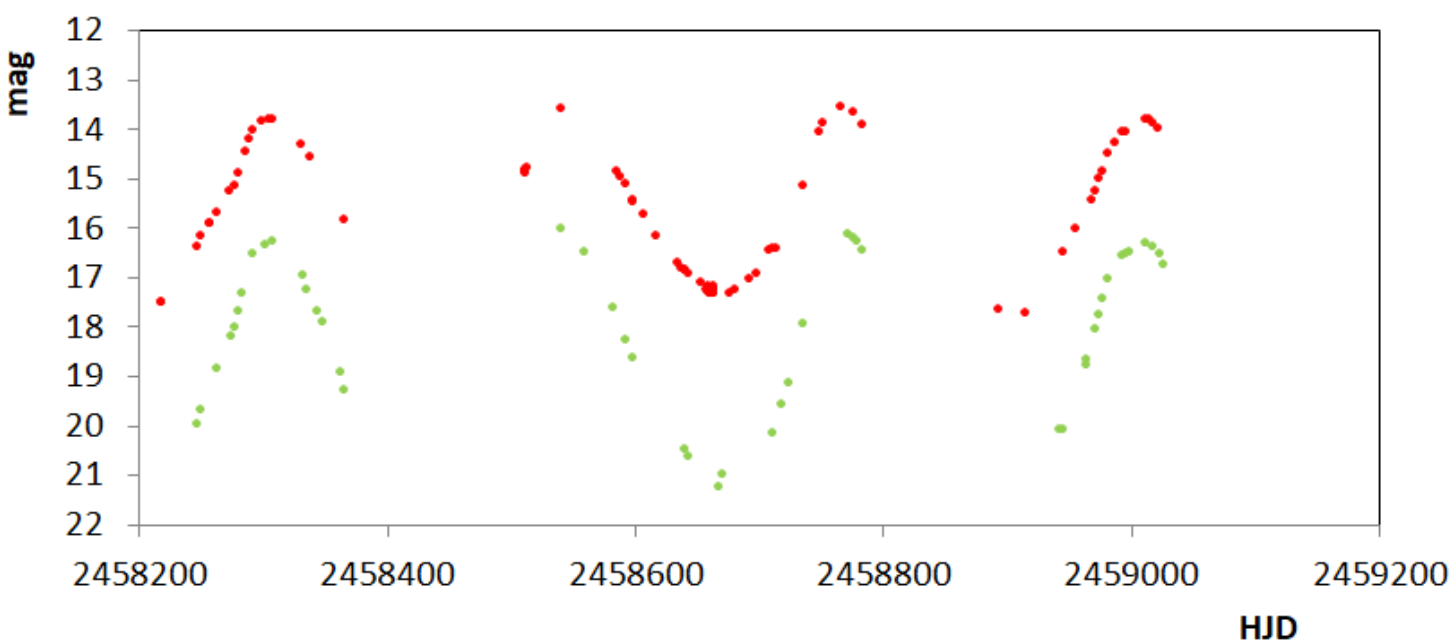

Figure 2.12 - Phase plot and JD light curve of ZTFJ175527.30-103939.8. Red and green dots denote ZTF $r$ and $g$ band data, respectively. The phase plot has been folded with $P=232 \mathrm{~d}$ (Table 1). 


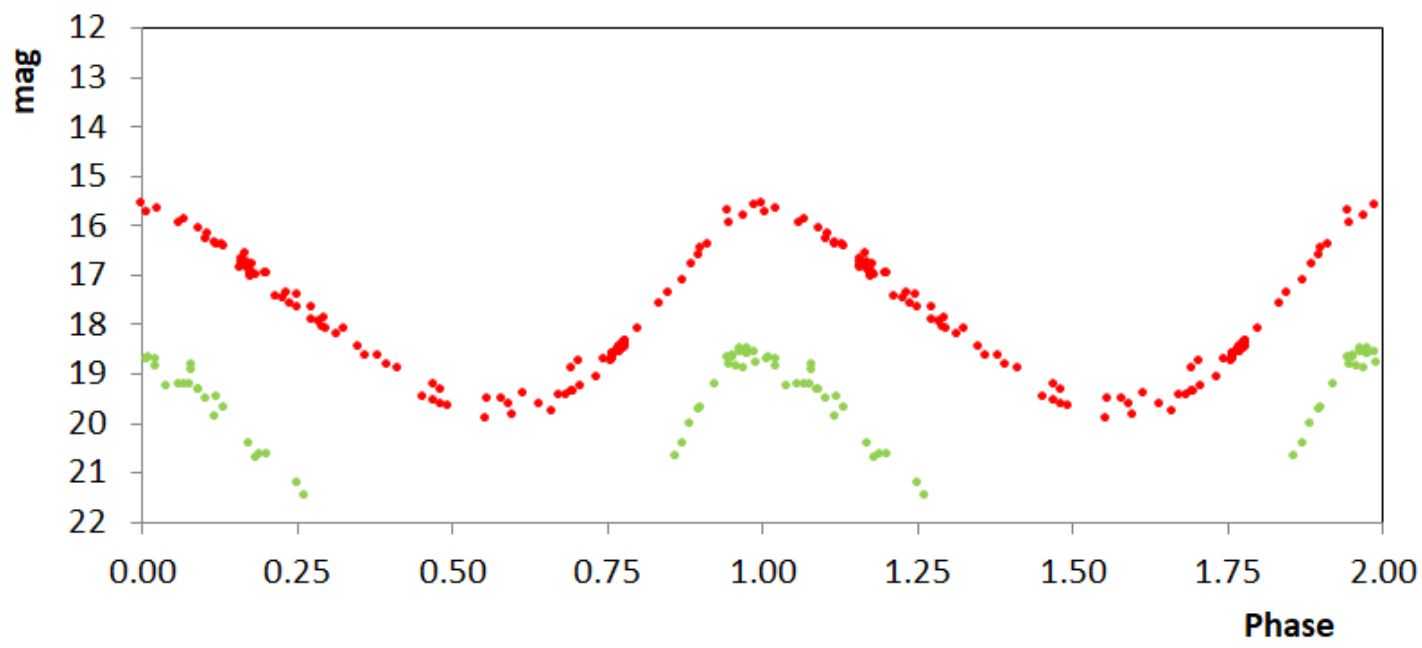

ZTFJ175924.69-052150.2

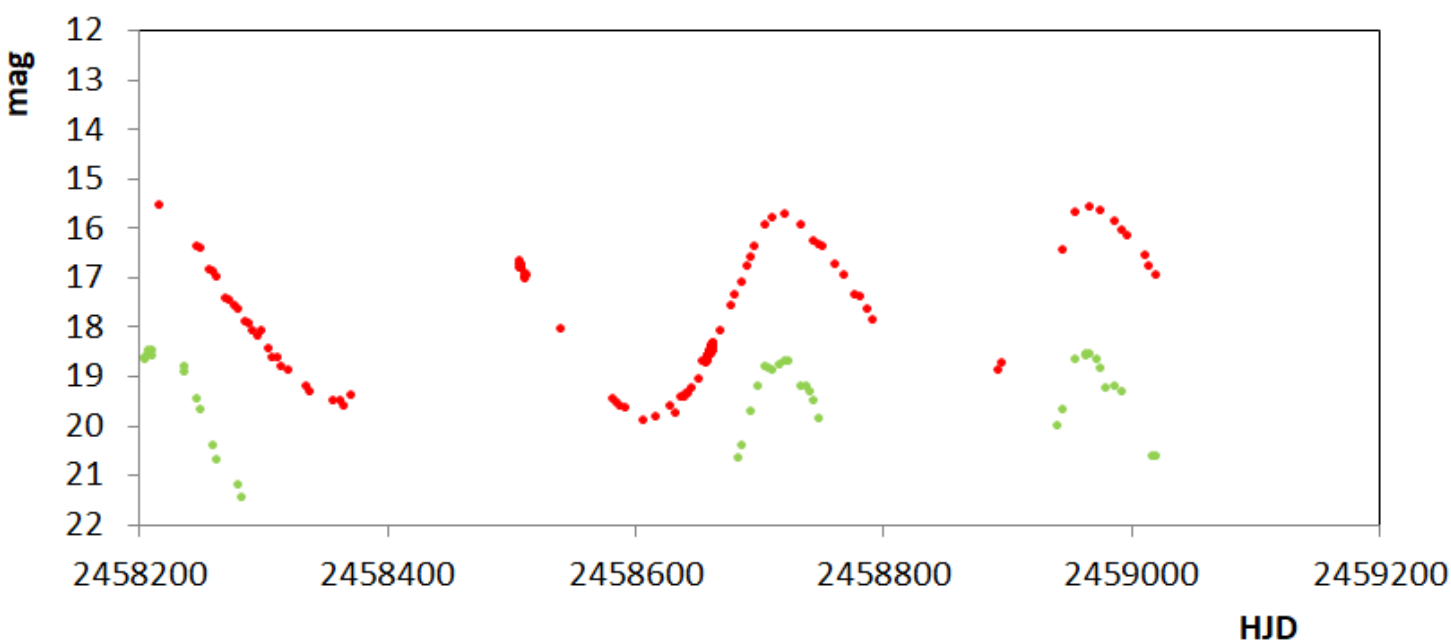

Figure 2.13 - Phase plot and JD light curve of ZTFJ175924.69-052150.2. Red and green dots denote ZTF $r$ and $g$ band data, respectively. The phase plot has been folded with $P=251 \mathrm{~d}$ (Table 1). 
ZTFJ180312.70-144808.1

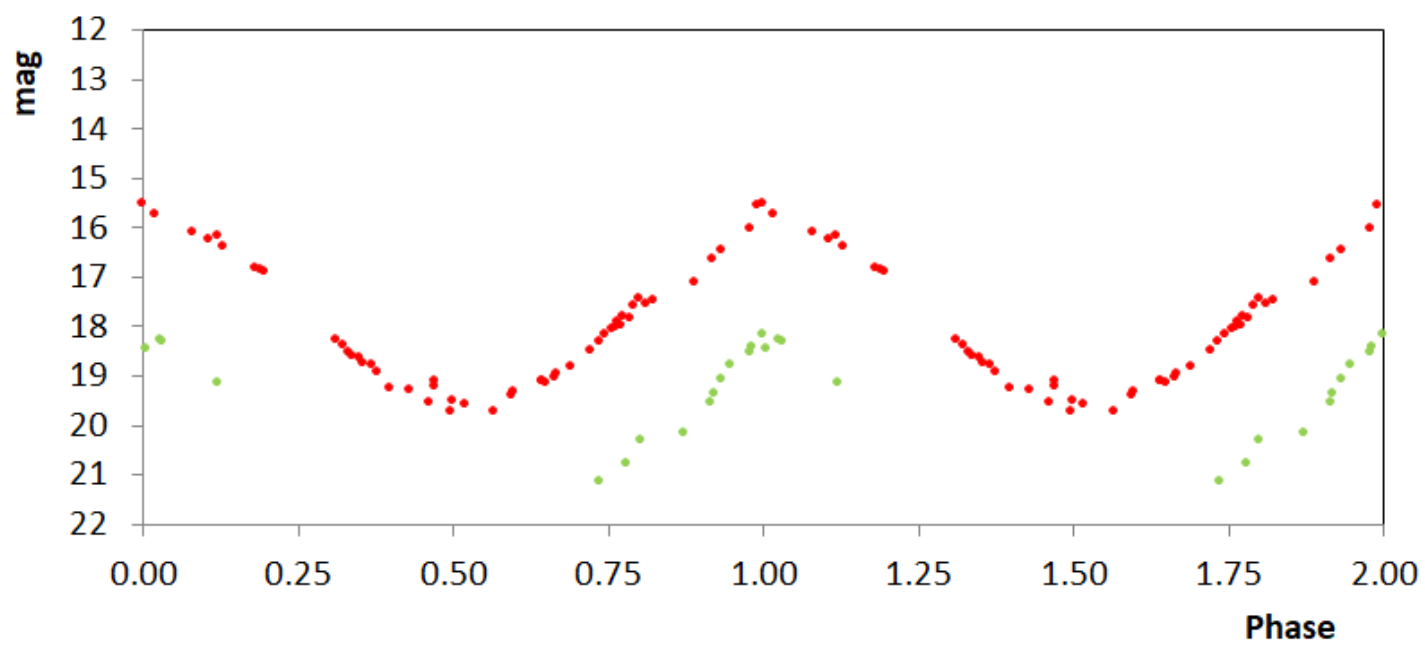

ZTFJ180312.70-144808.1

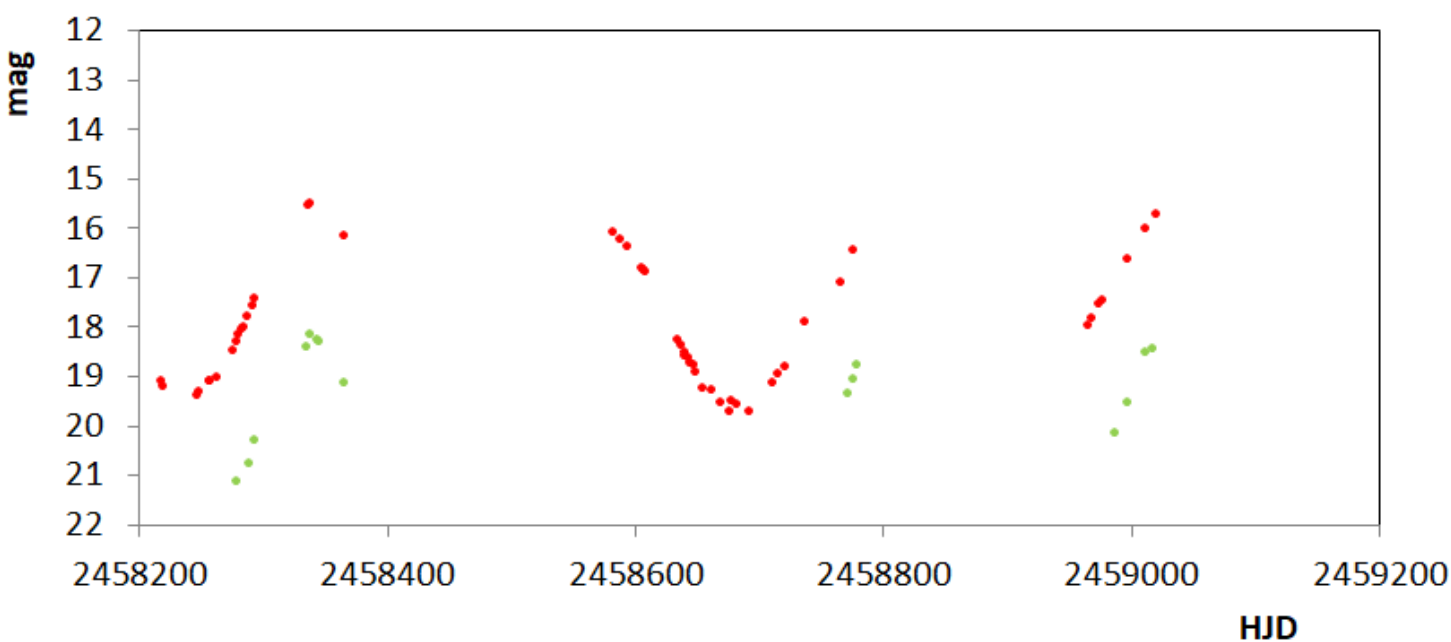

Figure 2.14 - Phase plot and JD light curve of ZTFJ180312.70-144808.1. Red and green dots denote ZTF $r$ and $g$ band data, respectively. The phase plot has been folded with $P=226 \mathrm{~d}$ (Table 1). 


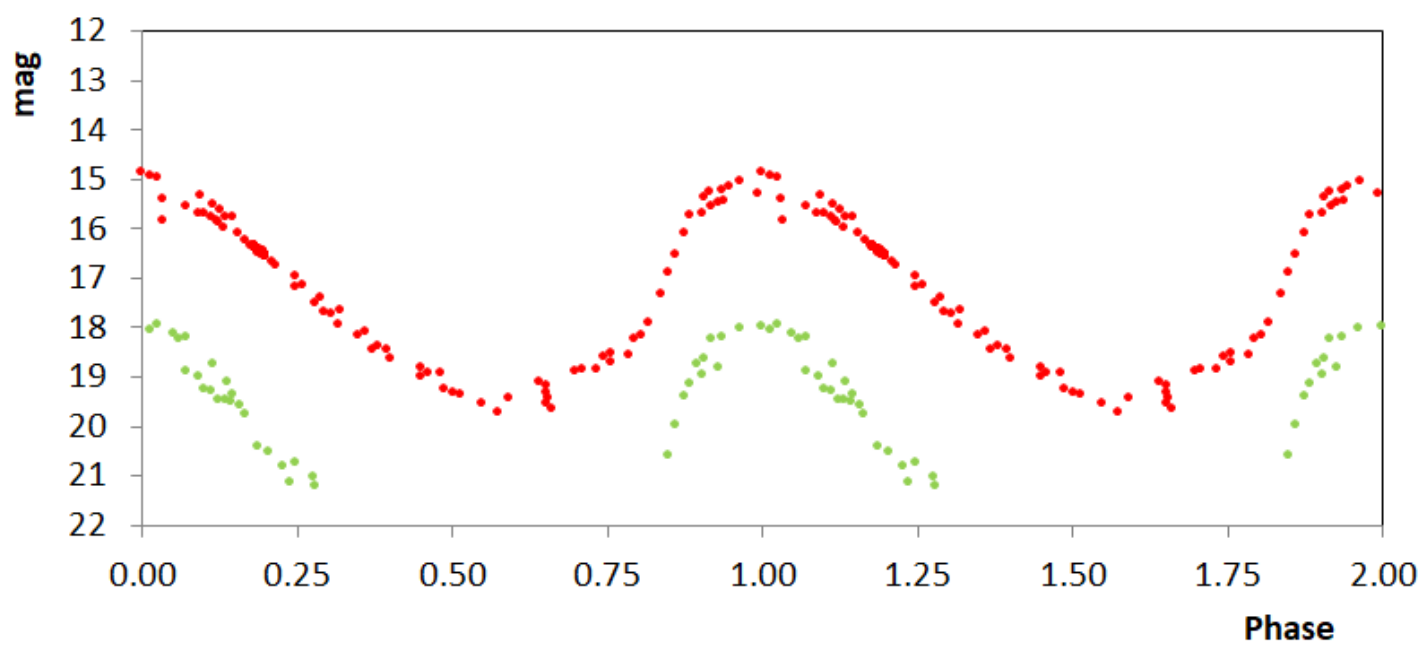

ZTFJ180329.13-040204.5

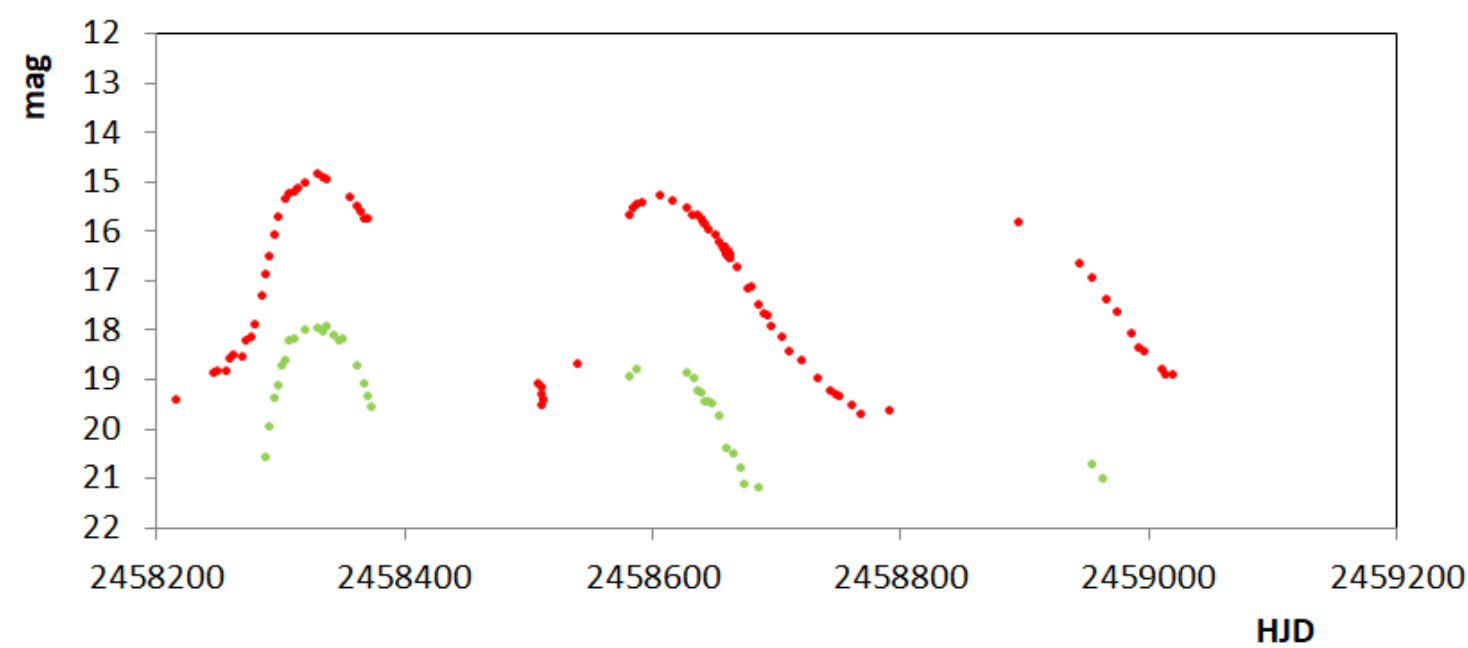

Figure 2.15 - Phase plot and JD light curve of ZTFJ180329.13-040204.5. Red and green dots denote ZTF $r$ and $g$ band data, respectively. The phase plot has been folded with $P=278 \mathrm{~d}$ (Table 1). 


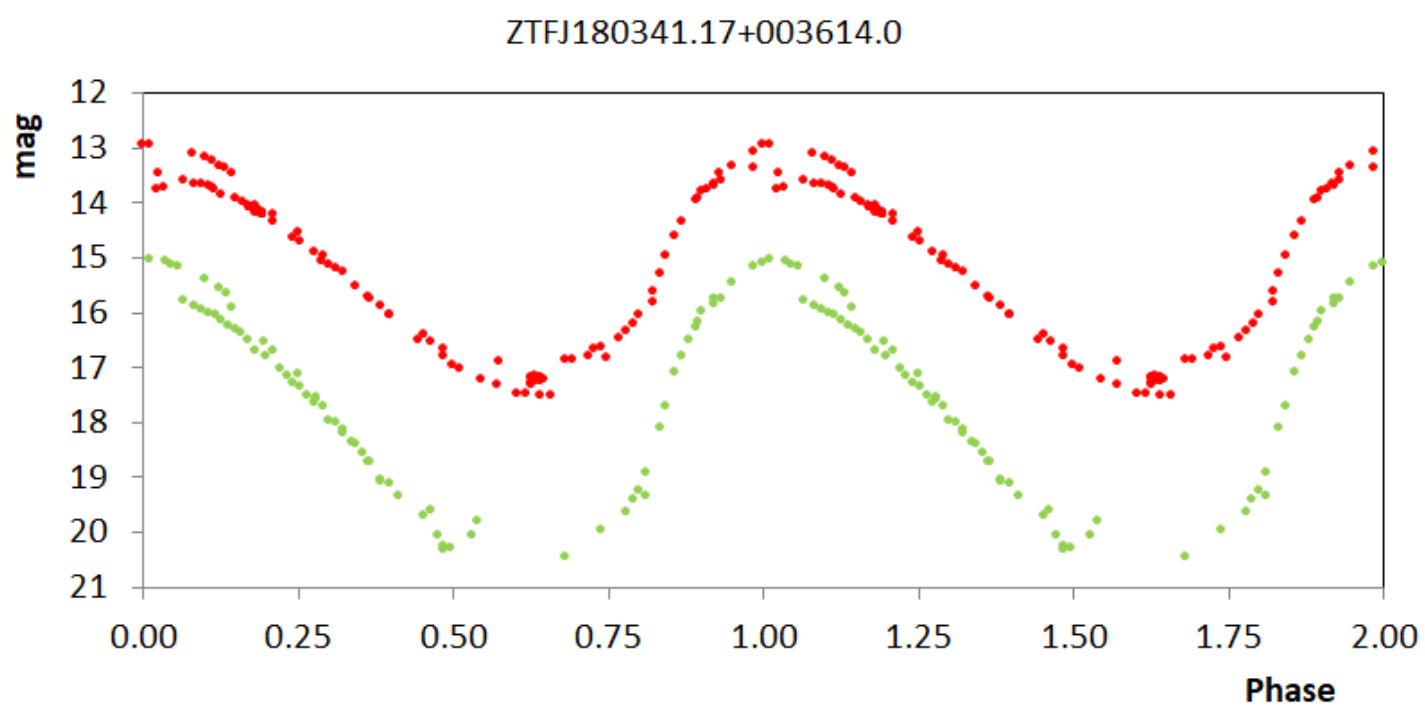

ZTFJ180341.17+003614.0

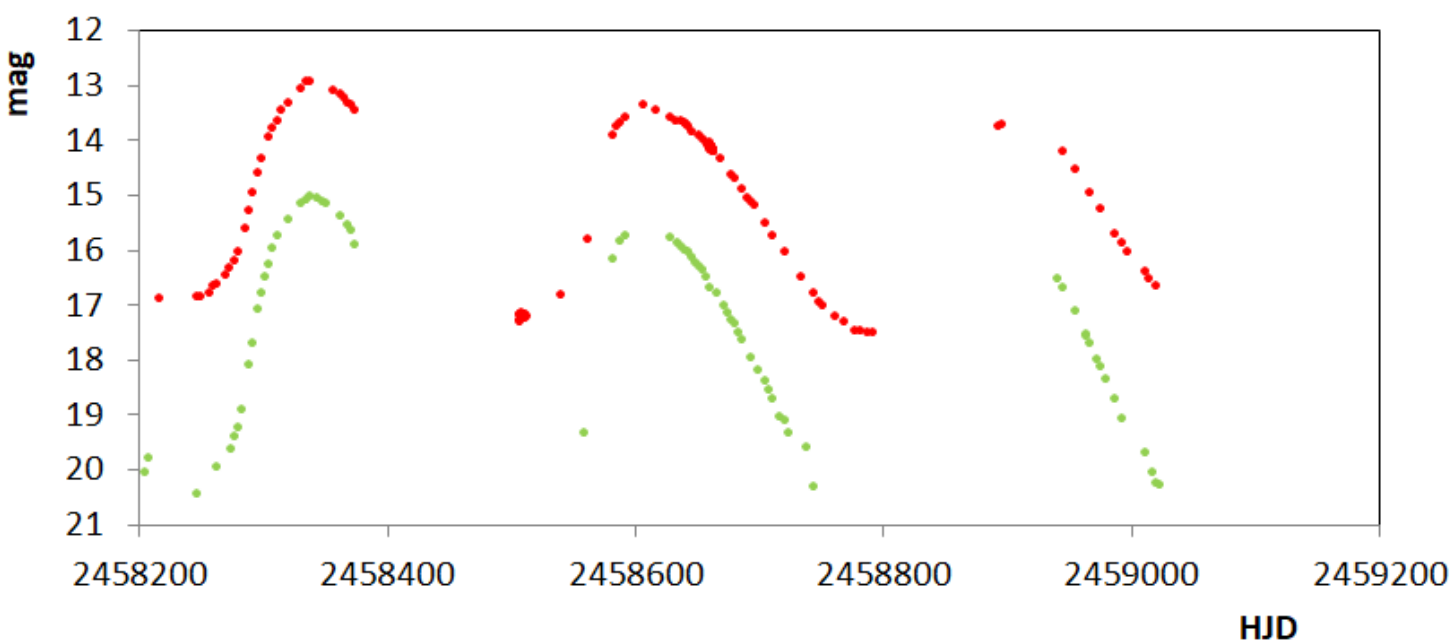

Figure 2.16 - Phase plot and JD light curve of ZTFJ180341.17+003614.0. Red and green dots denote ZTF $r$ and $g$ band data, respectively. The phase plot has been folded with $P=276 \mathrm{~d}$ (Table 1). 


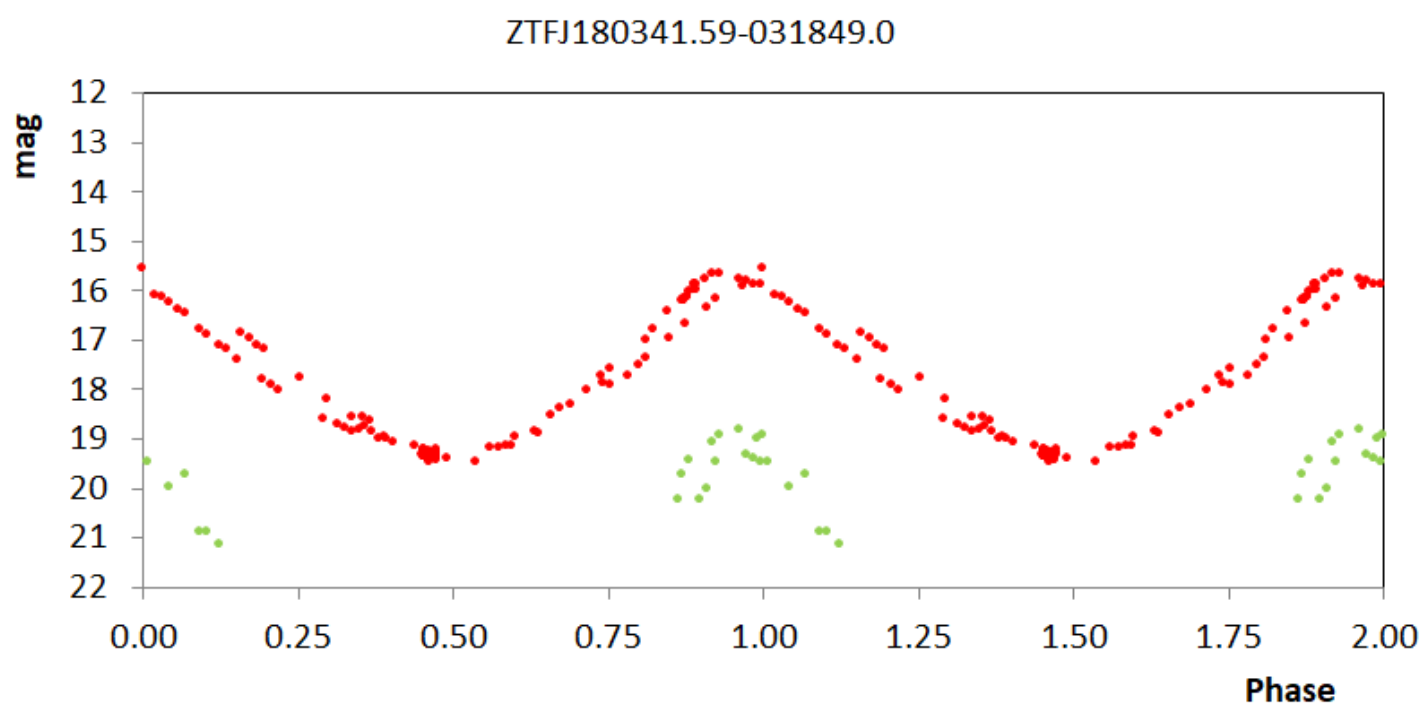

ZTFJ180341.59-031849.0

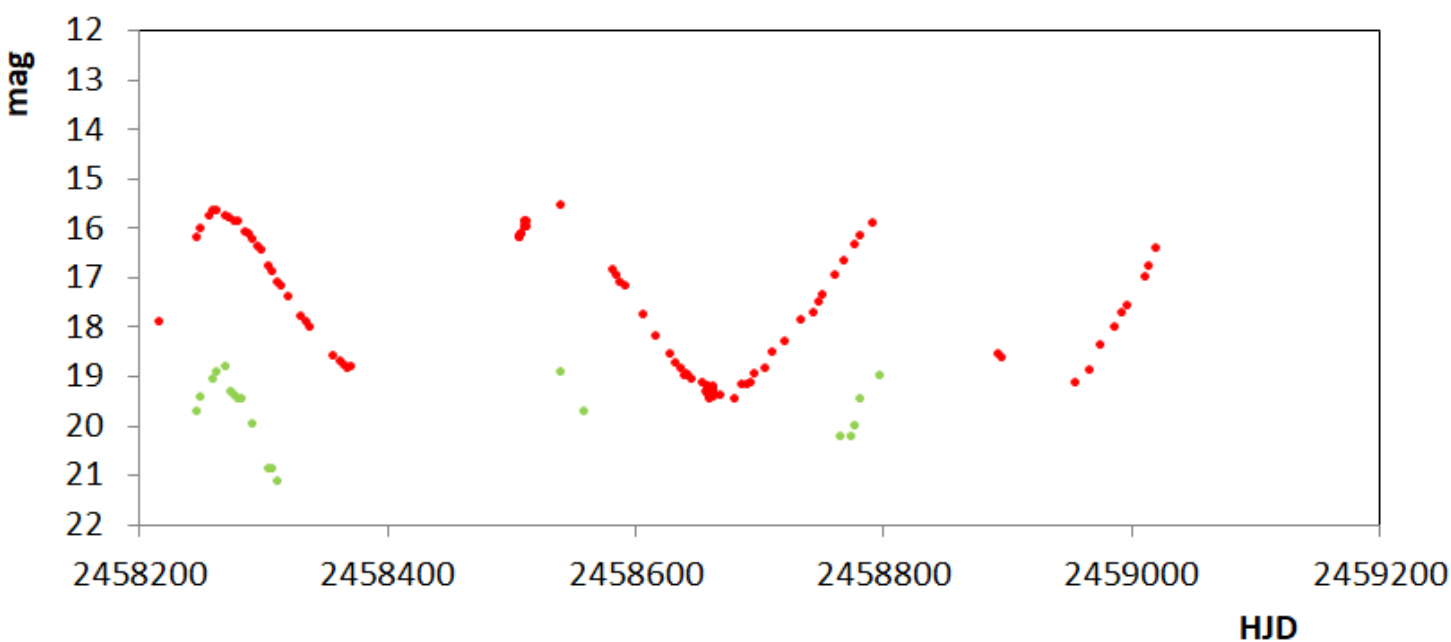

Figure 2.17 - Phase plot and JD light curve of ZTFJ180341.59-031849.0. Red and green dots denote ZTF $r$ and $g$ band data, respectively. The phase plot has been folded with $P=260 \mathrm{~d}$ (Table 1). 


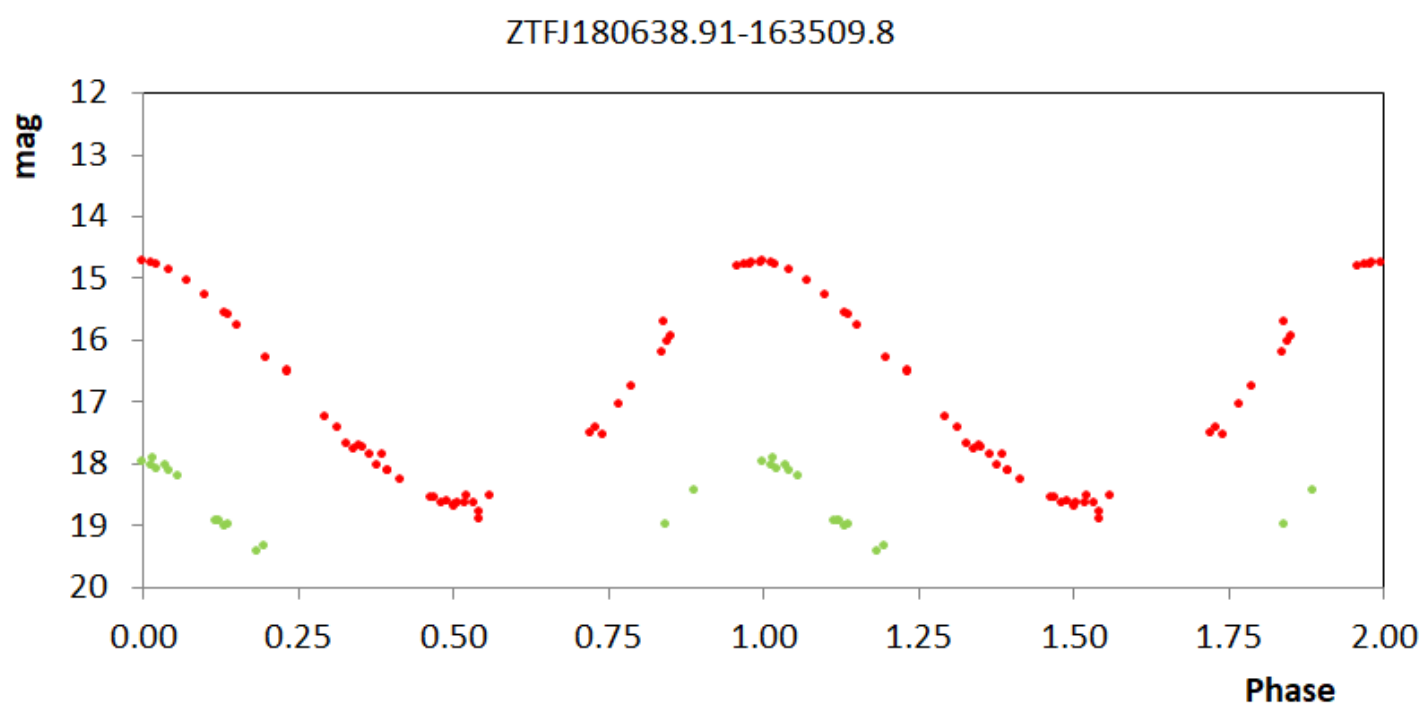

ZTFJ180638.91-163509.8

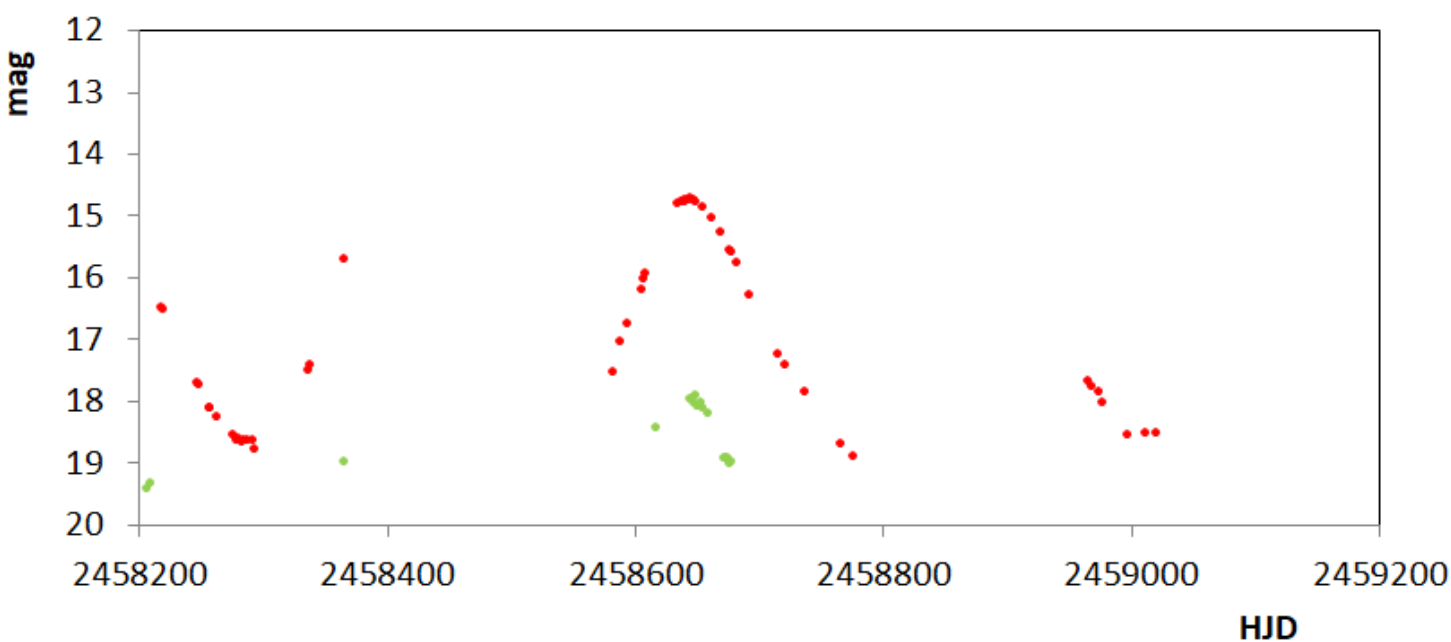

Figure 2.18 - Phase plot and JD light curve of ZTFJ180638.91-163509.8. Red and green dots denote ZTF $r$ and $g$ band data, respectively. The phase plot has been folded with $P=241 \mathrm{~d}$ (Table 1). 
ZTFJ180640.24-135159.8

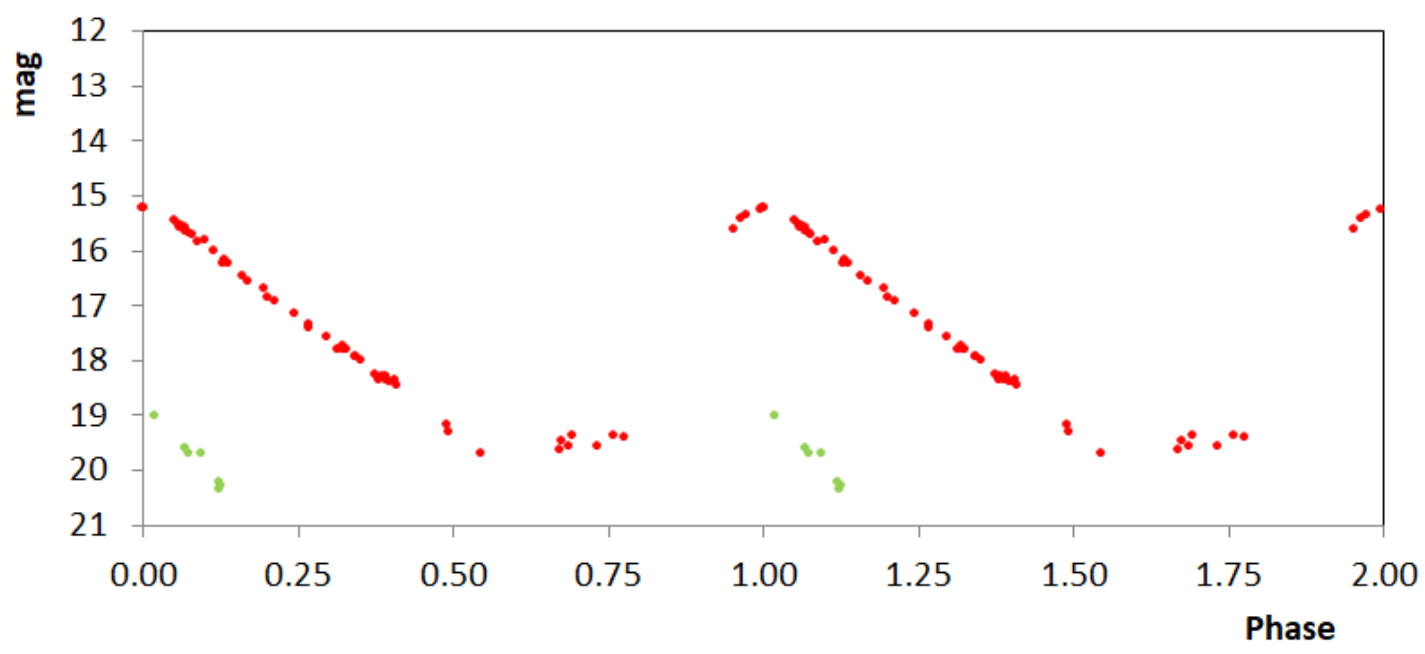

ZTFJ180640.24-135159.8

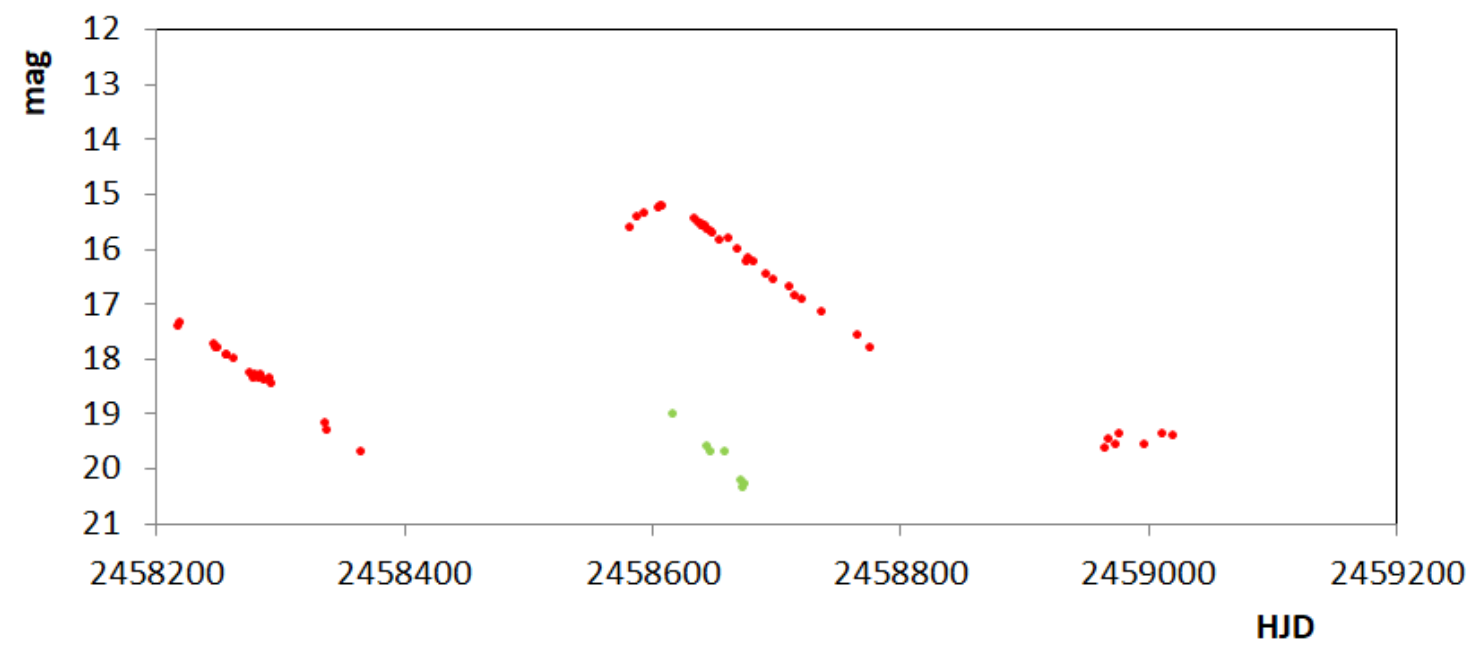

Figure 2.19 - Phase plot and JD light curve of ZTFJ180640.24-135159.8. Red and green dots denote ZTF $r$ and $g$ band data, respectively. The phase plot has been folded with $P=532 \mathrm{~d}$ (Table 1). 


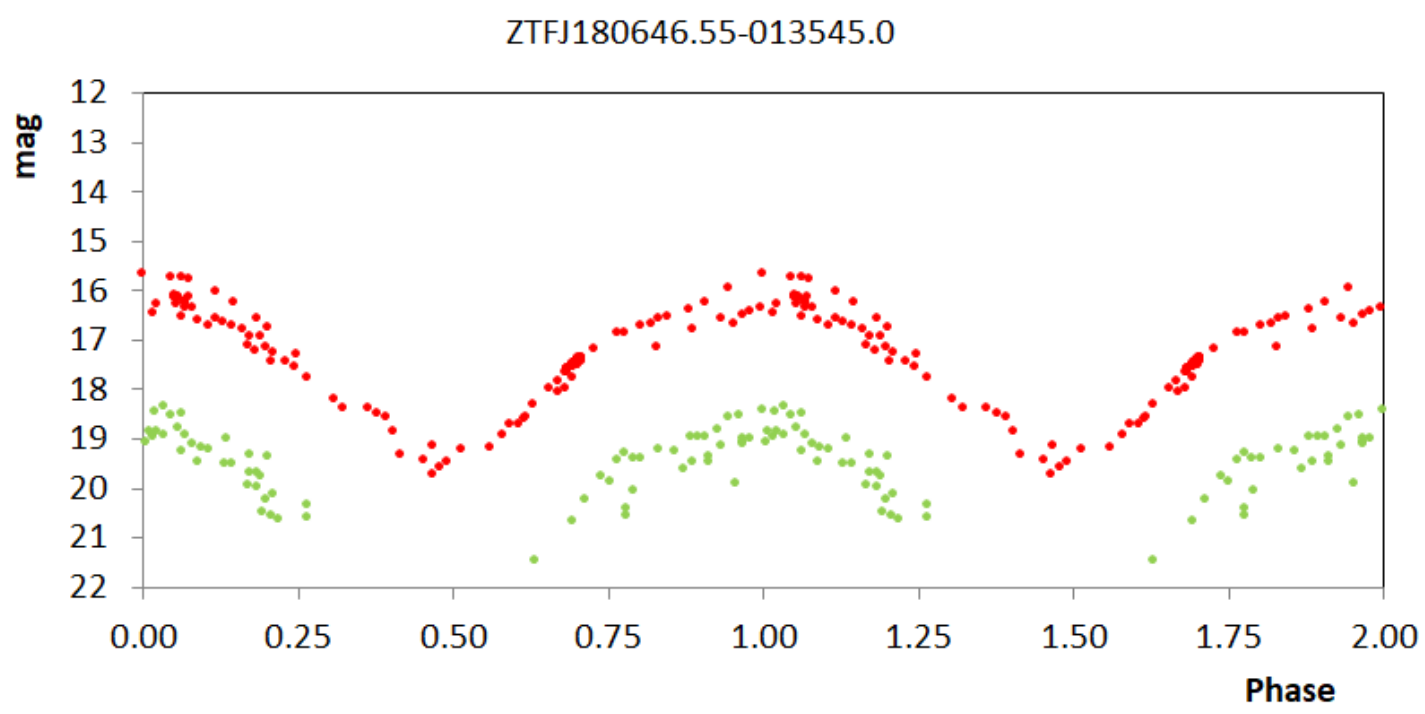

ZTFJ180646.55-013545.0

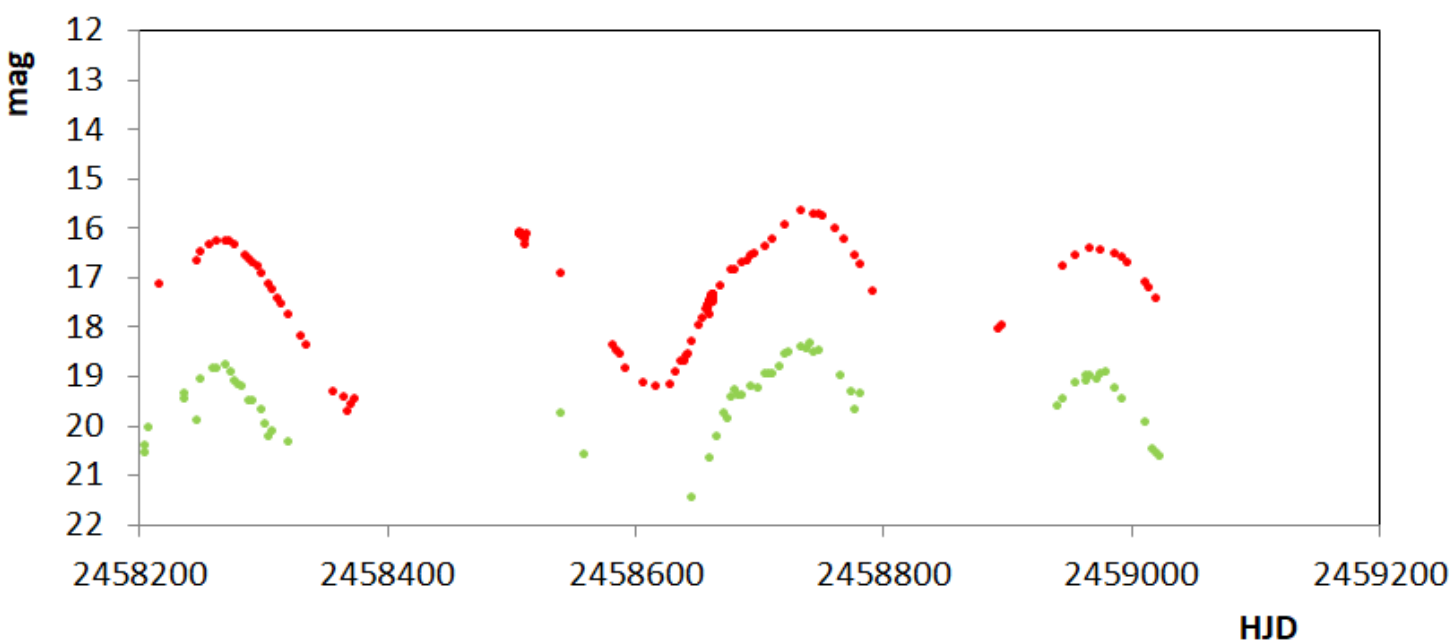

Figure 2.20 - Phase plot and JD light curve of ZTFJ180646.55-013545.0. Red and green dots denote ZTF $r$ and $g$ band data, respectively. The phase plot has been folded with $P=238 \mathrm{~d}$ (Table 1). 
ZTFJ180729.85-013438.1

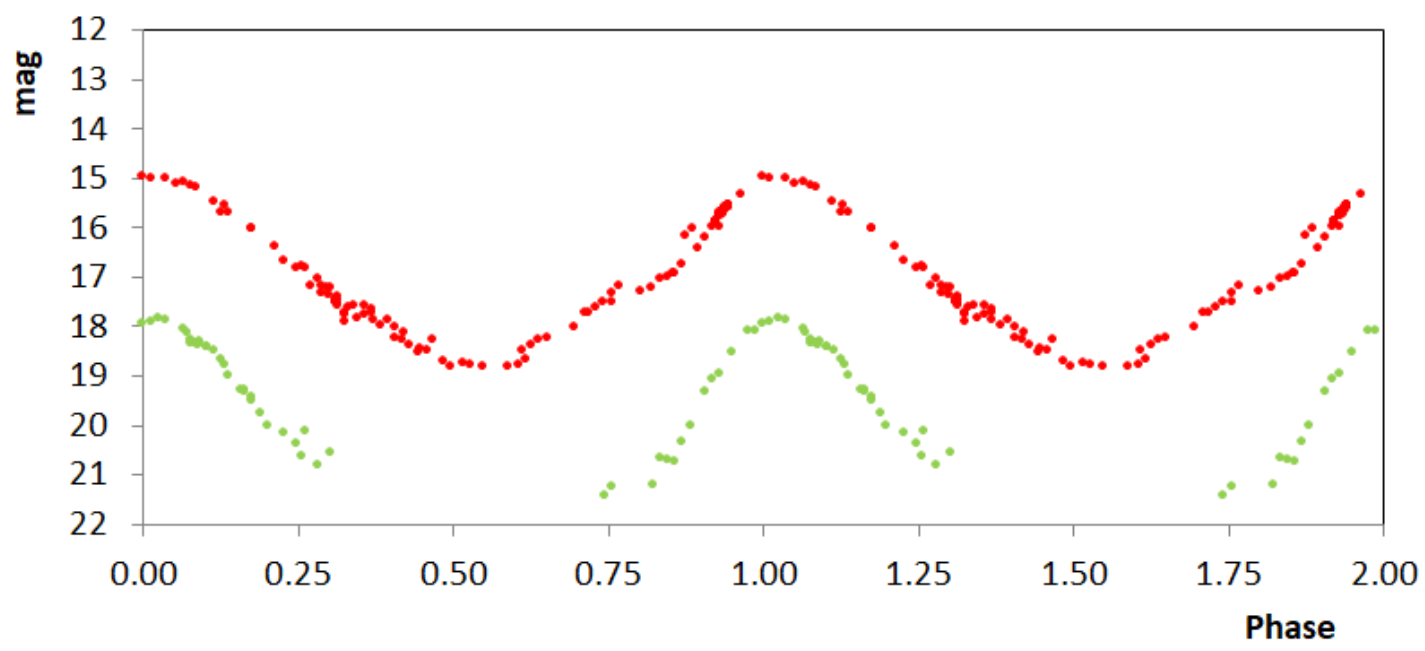

ZTFJ180729.85-013438.1

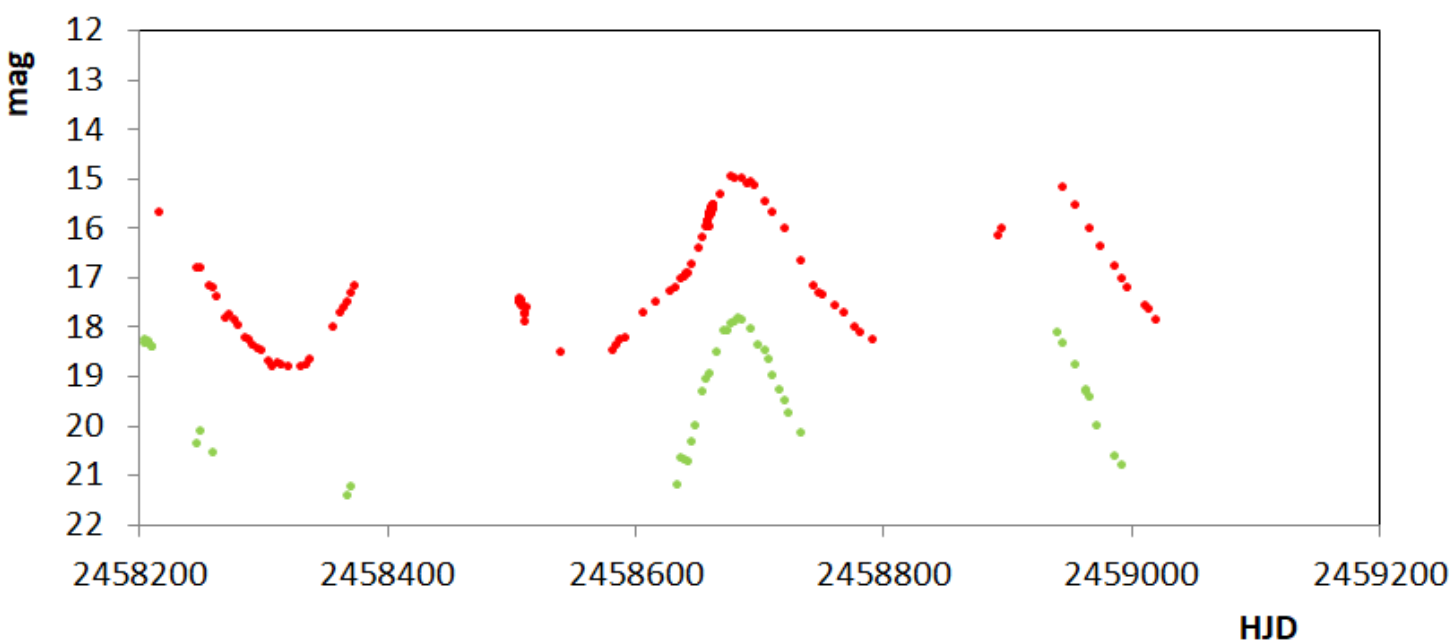

Figure 2.21 - Phase plot and JD light curve of ZTFJ180729.85-013438.1. Red and green dots denote ZTF $r$ and $g$ band data, respectively. The phase plot has been folded with $P=246 \mathrm{~d}$ (Table 1). 
ZTFJ180826.00-174129.0

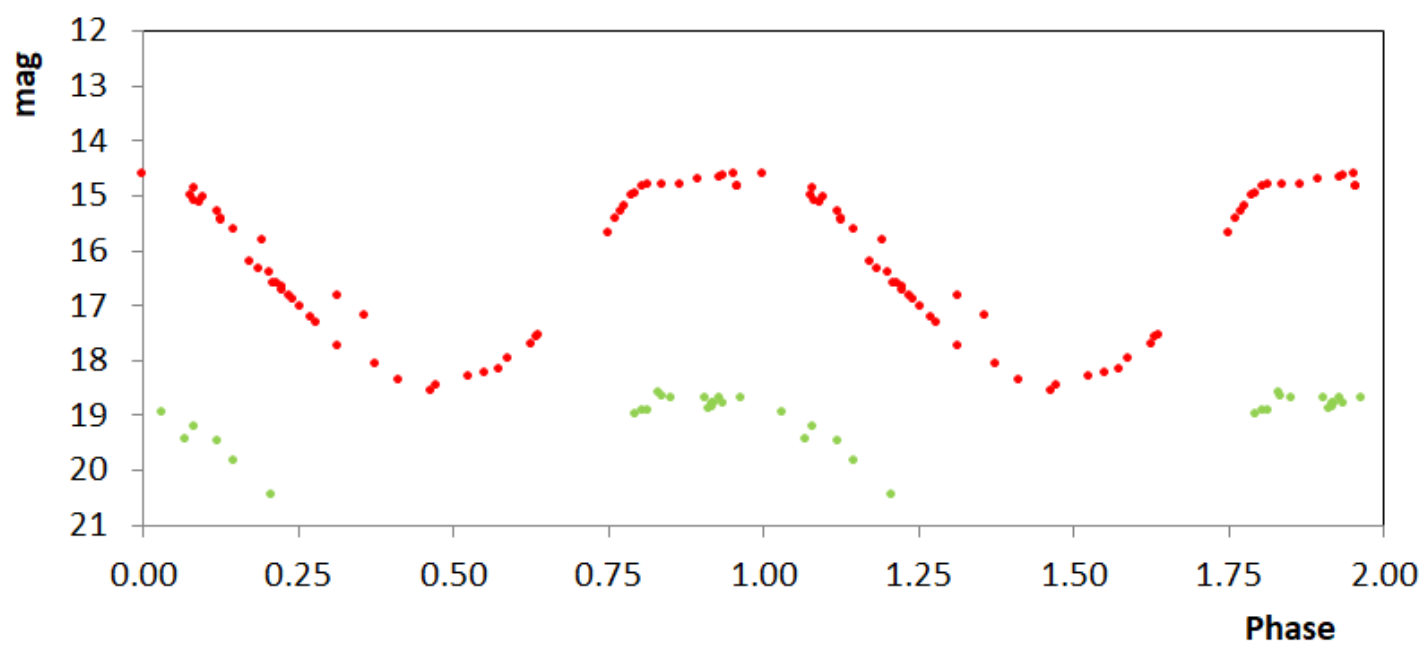

ZTFJ180826.00-174129.0

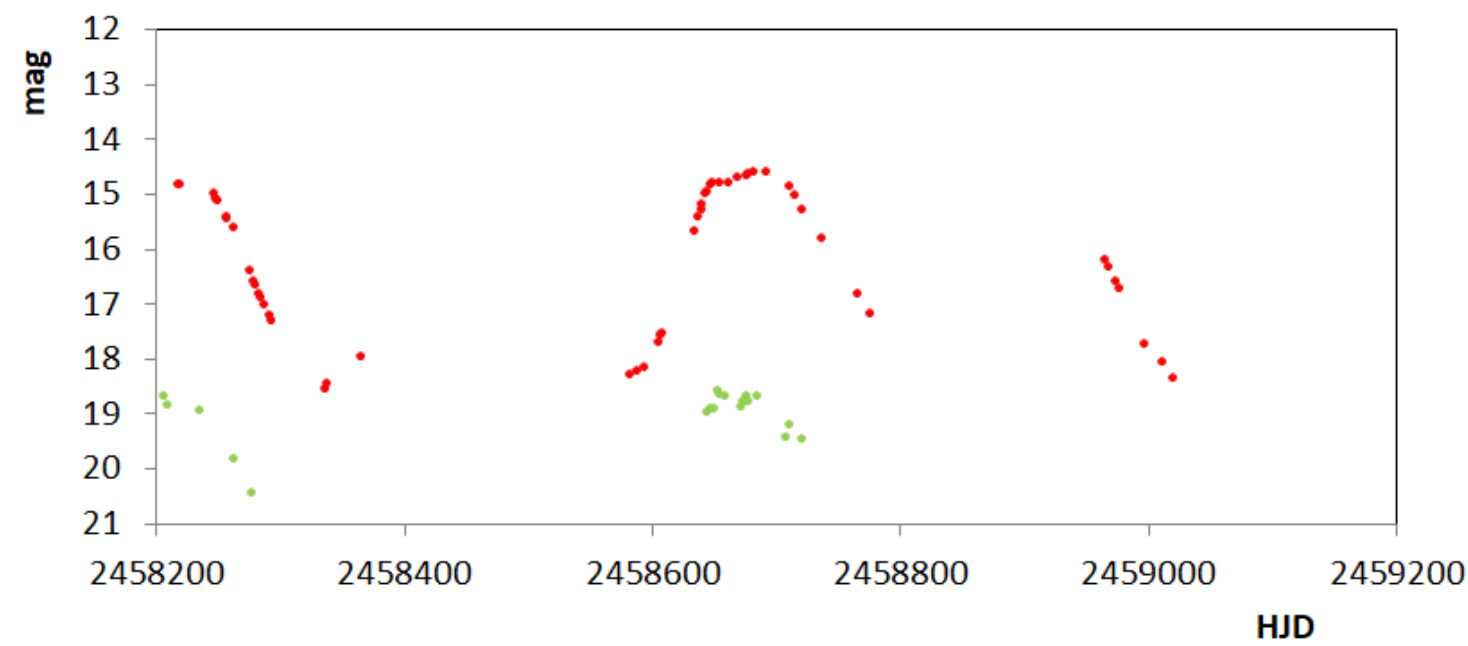

Figure 2.22 - Phase plot and JD light curve of ZTFJ180826.00-174129.0. Red and green dots denote ZTF $r$ and $g$ band data, respectively. The phase plot has been folded with $P=232 \mathrm{~d}$ (Table 1). 
ZTFJ184503.44+154335.3

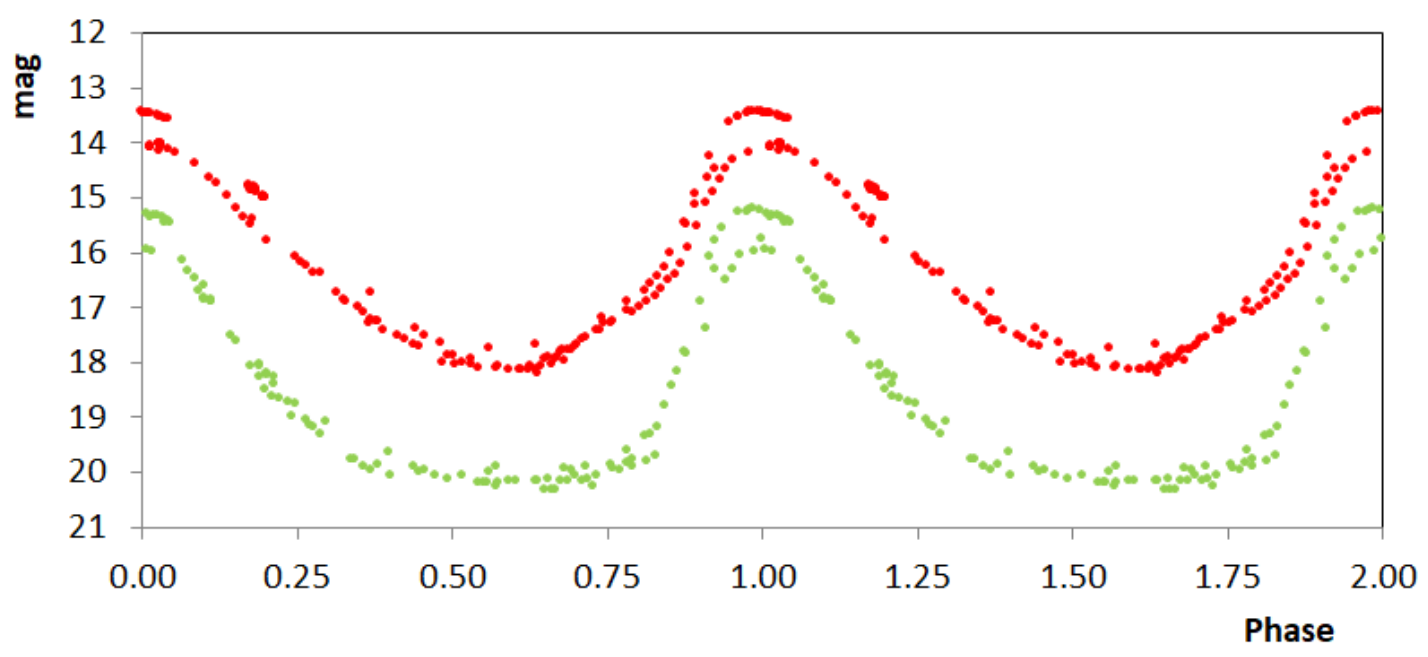

ZTFJ184503.44+154335.3

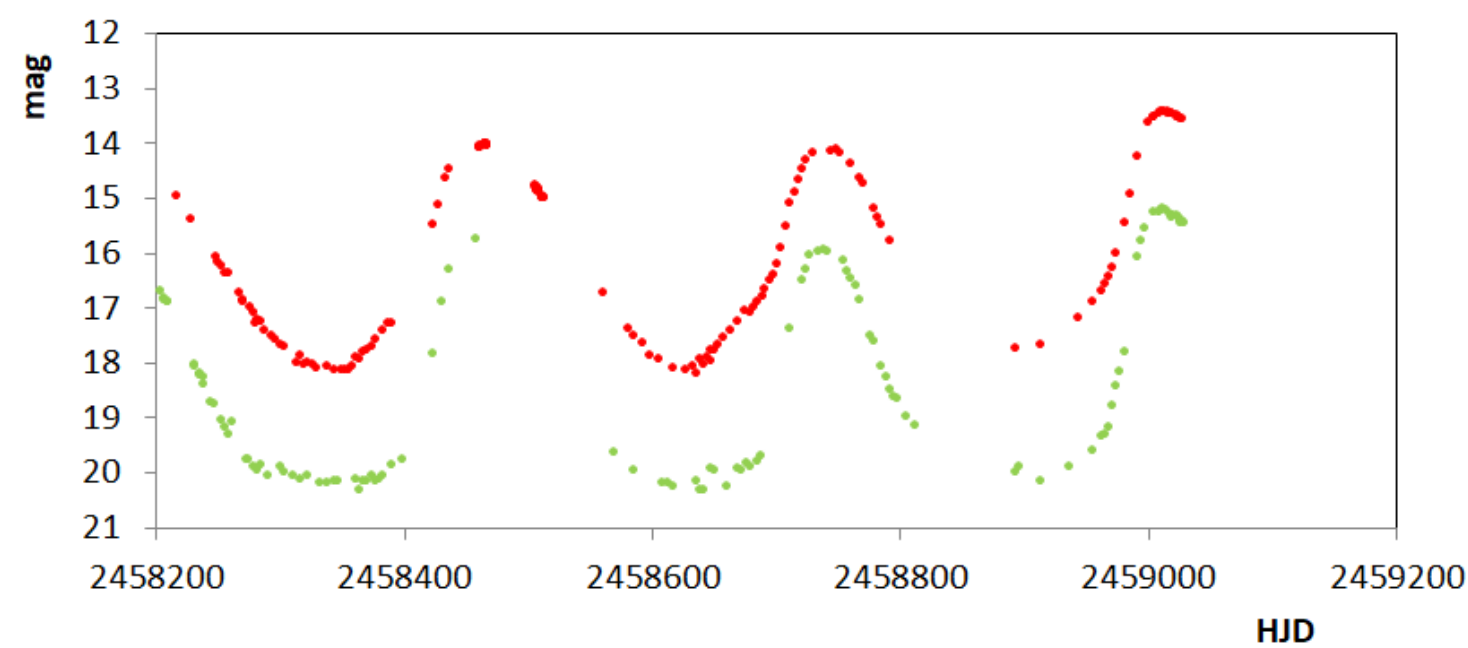

Figure 2.23 - Phase plot and JD light curve of ZTFJ184503.44+154335.3. Red and green dots denote ZTF $r$ and $g$ band data, respectively. The phase plot has been folded with $P=279 \mathrm{~d}$ (Table 1). 
ZTFJ190648.43+130203.5

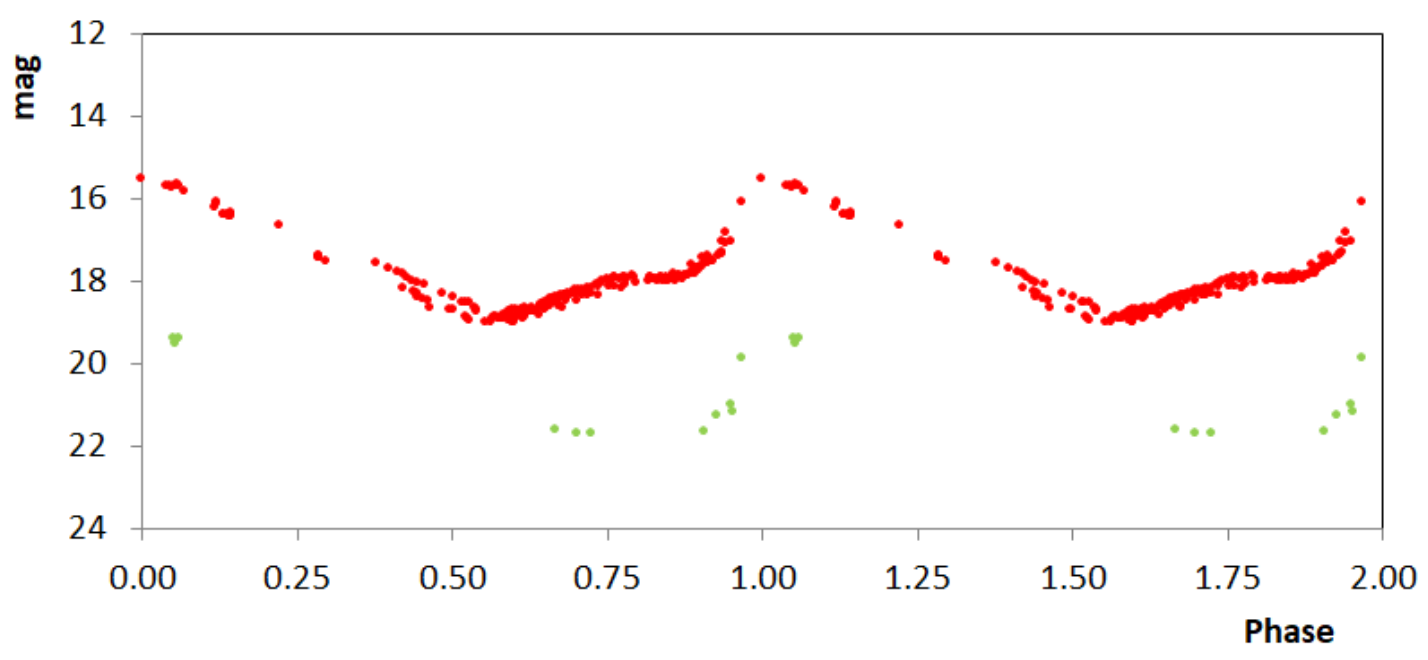

ZTFJ190648.43+130203.5

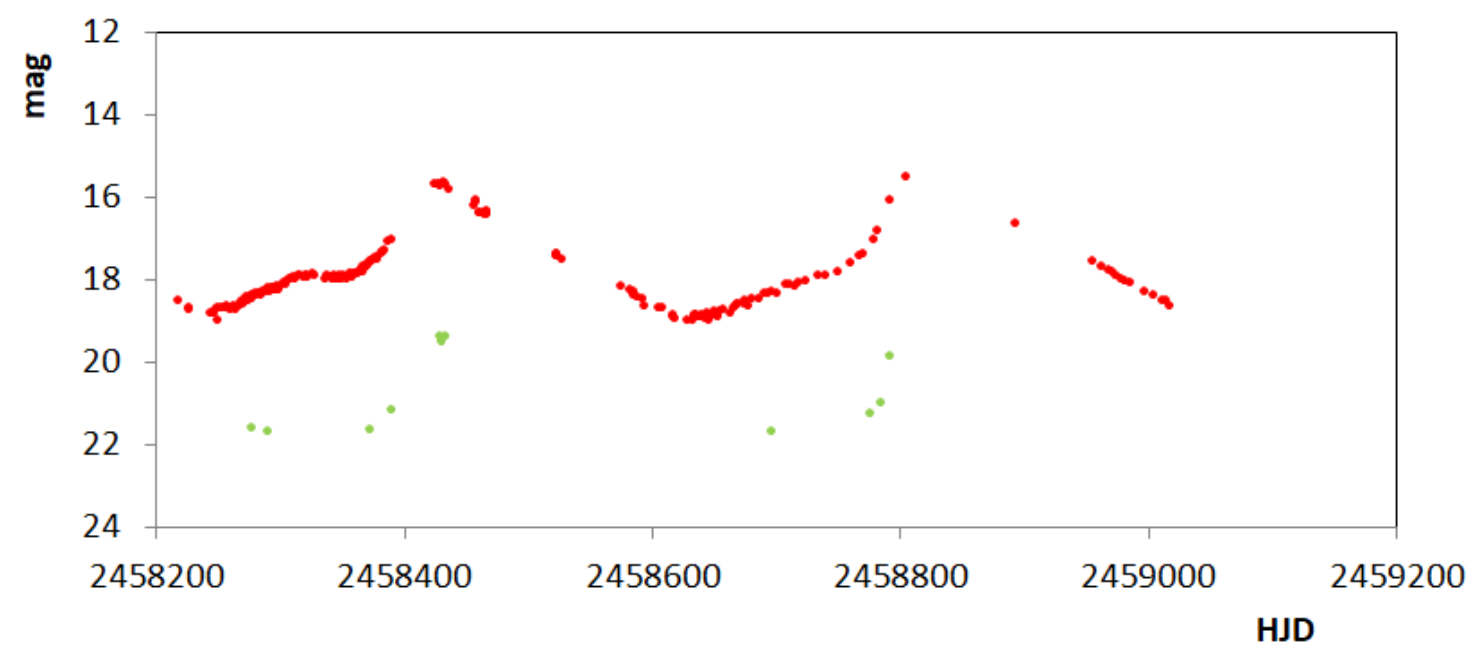

Figure 2.24 - Phase plot and JD light curve of ZTFJ190648.43+130203.5. Red and green dots denote ZTF $r$ and $g$ band data, respectively. The phase plot has been folded with $P=396 \mathrm{~d}$ (Table 1). 
ZTFJ191008.27+131014.4

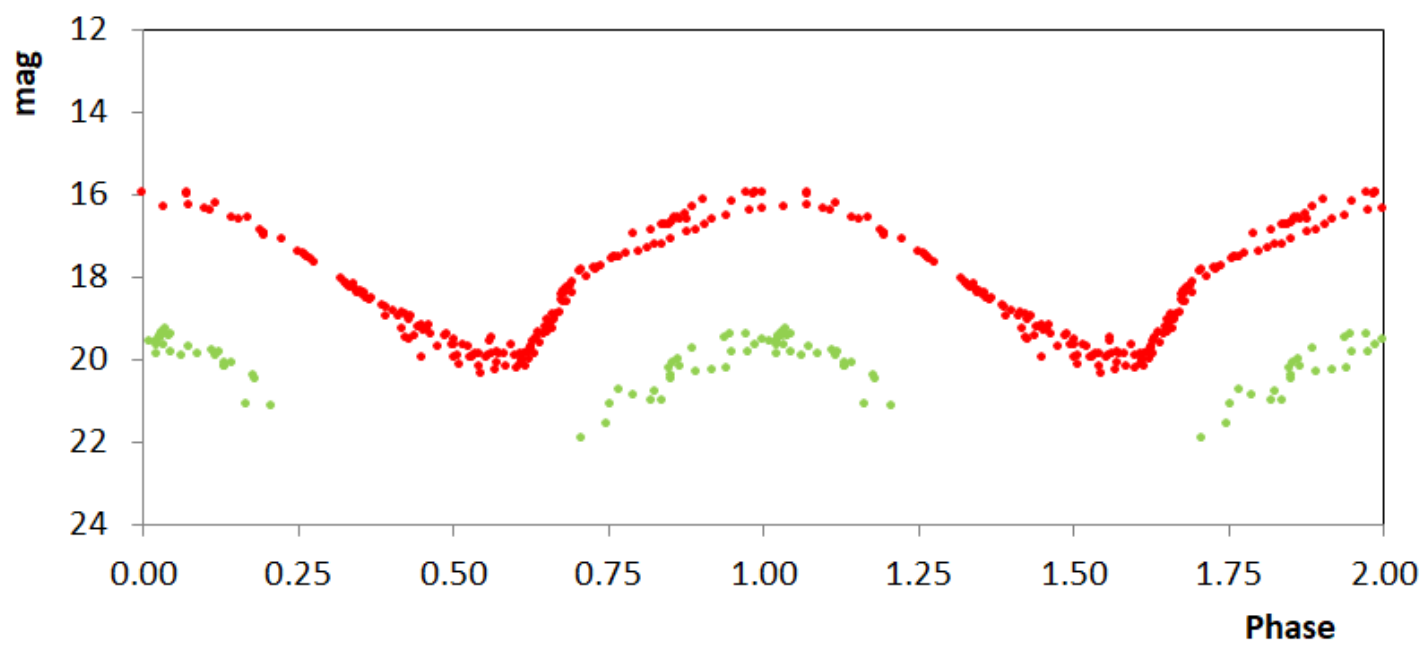

ZTFJ191008.27+131014.4

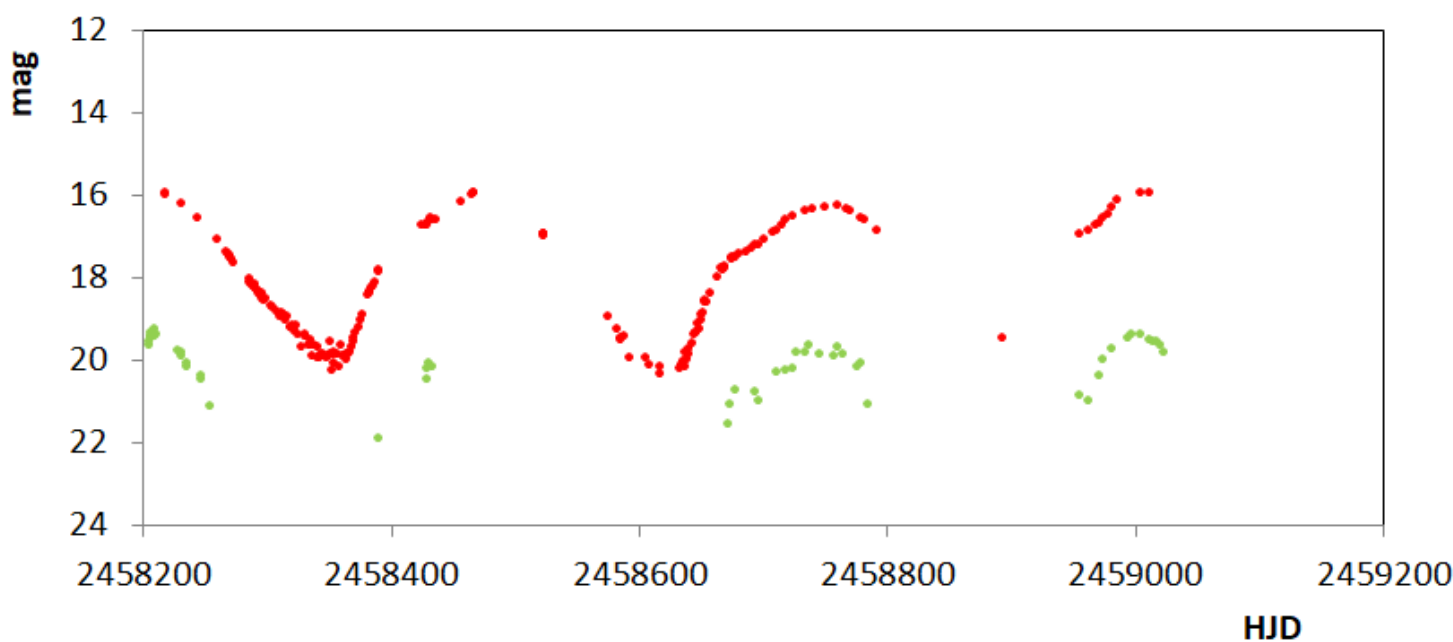

Figure 2.25 - Phase plot and JD light curve of ZTFJ191008.27+131014.4. Red and green dots denote ZTF $r$ and $g$ band data, respectively. The phase plot has been folded with $P=271 \mathrm{~d}$ (Table 1). 


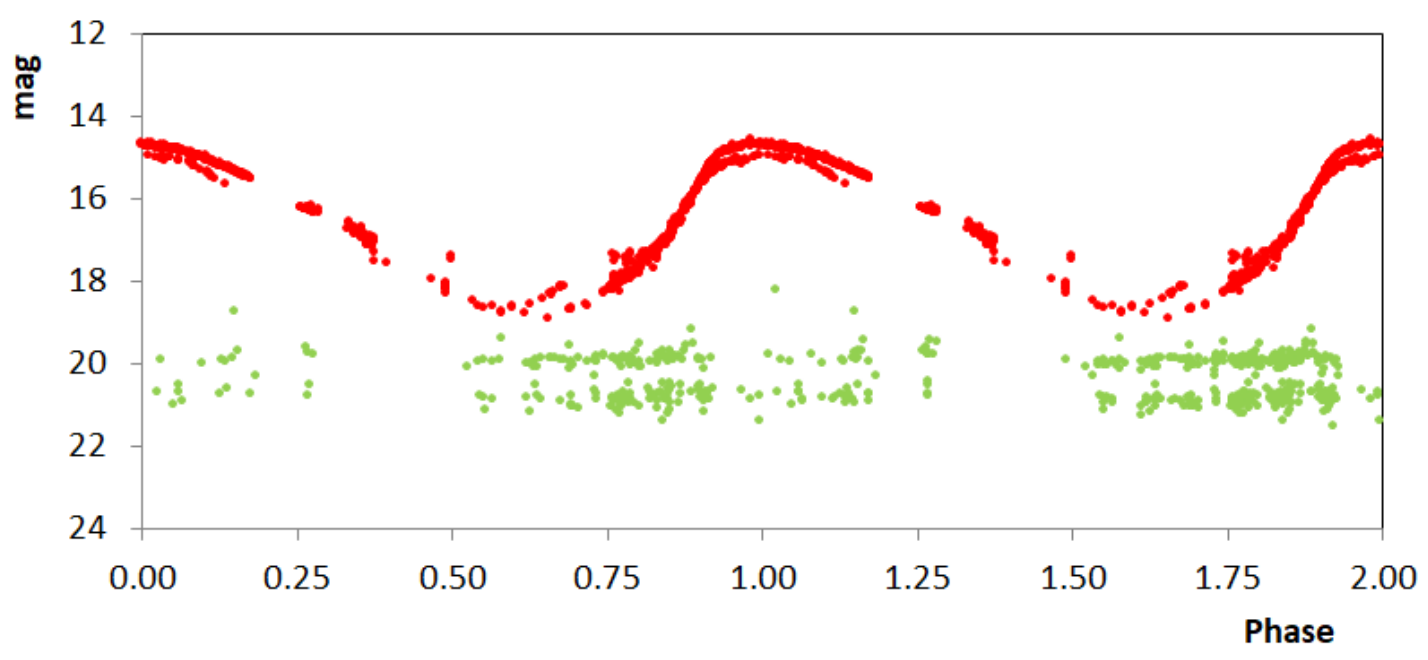

ZTFJ191406.43+151810.6

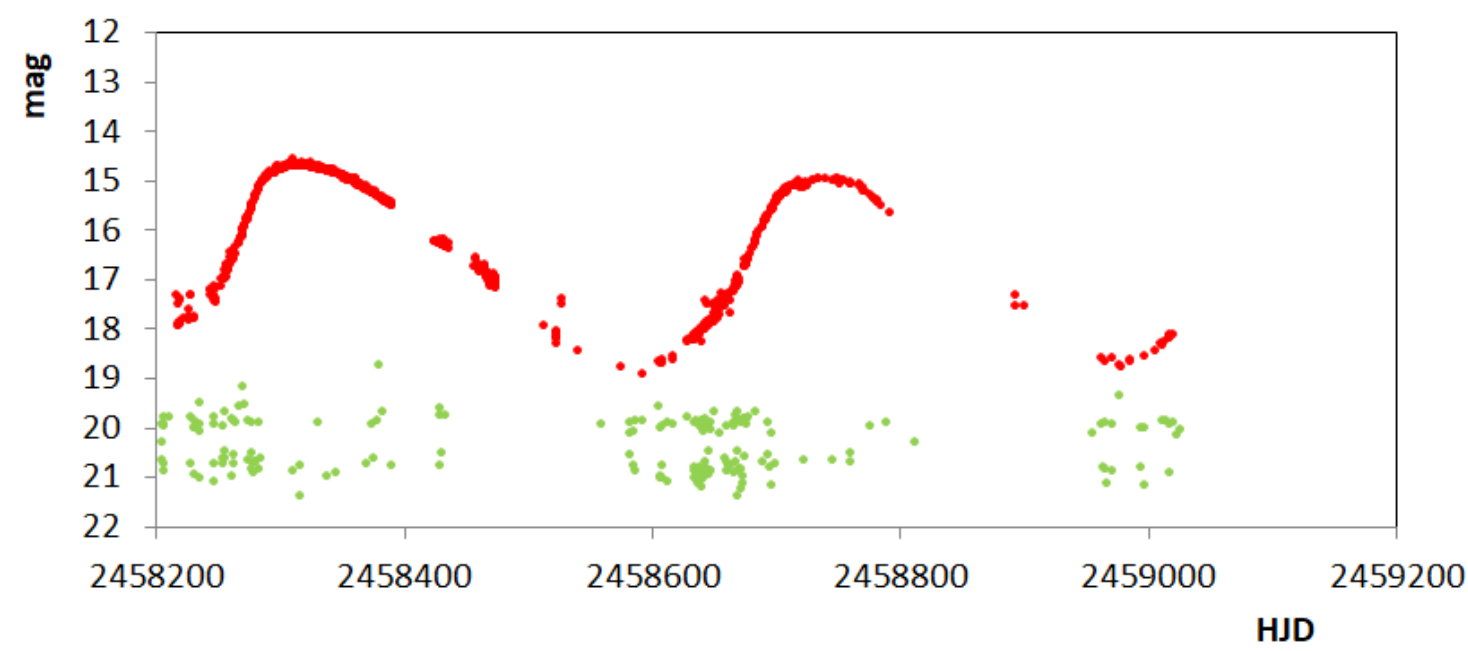

Figure 2.26 - Phase plot and JD light curve of ZTFJ191406.43+151810.6. Red and green dots denote ZTF $r$ and $g$ band data, respectively. The phase plot has been folded with $P=418 \mathrm{~d}$ (Table 1). 


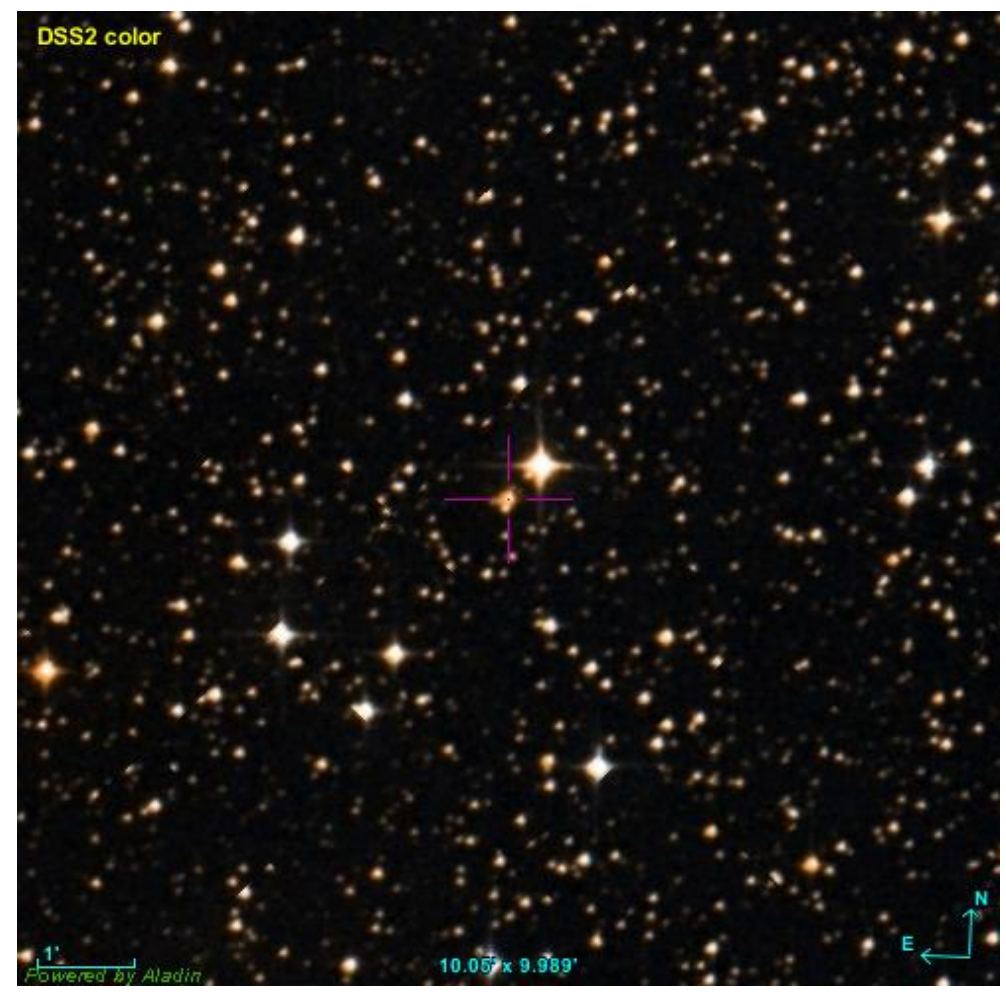

Figure 3.1 Sky region of ZTFJ070812.40-194557.1 (field of view 10.05' x 9.989').

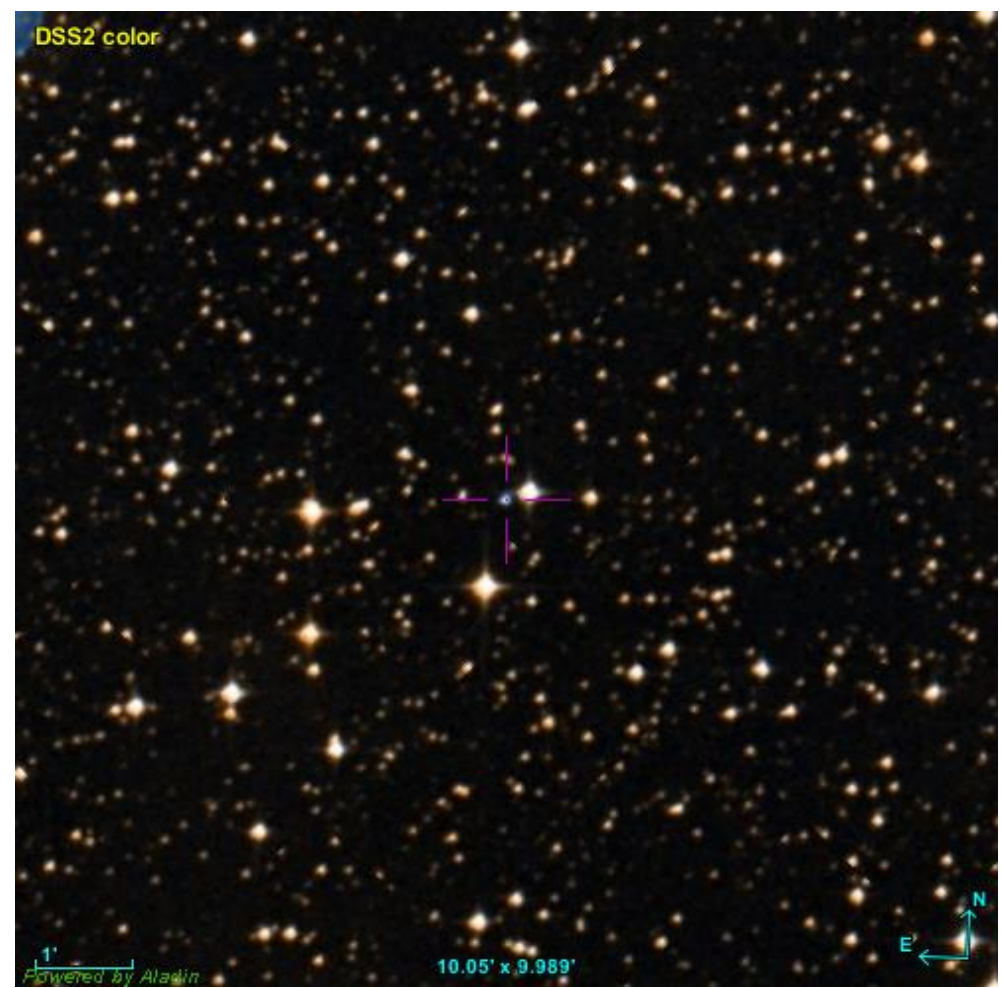

Figure 3.2 Sky region of ZTFJ070912.44-191159.4 (field of view 10.05' x 9.989'). 


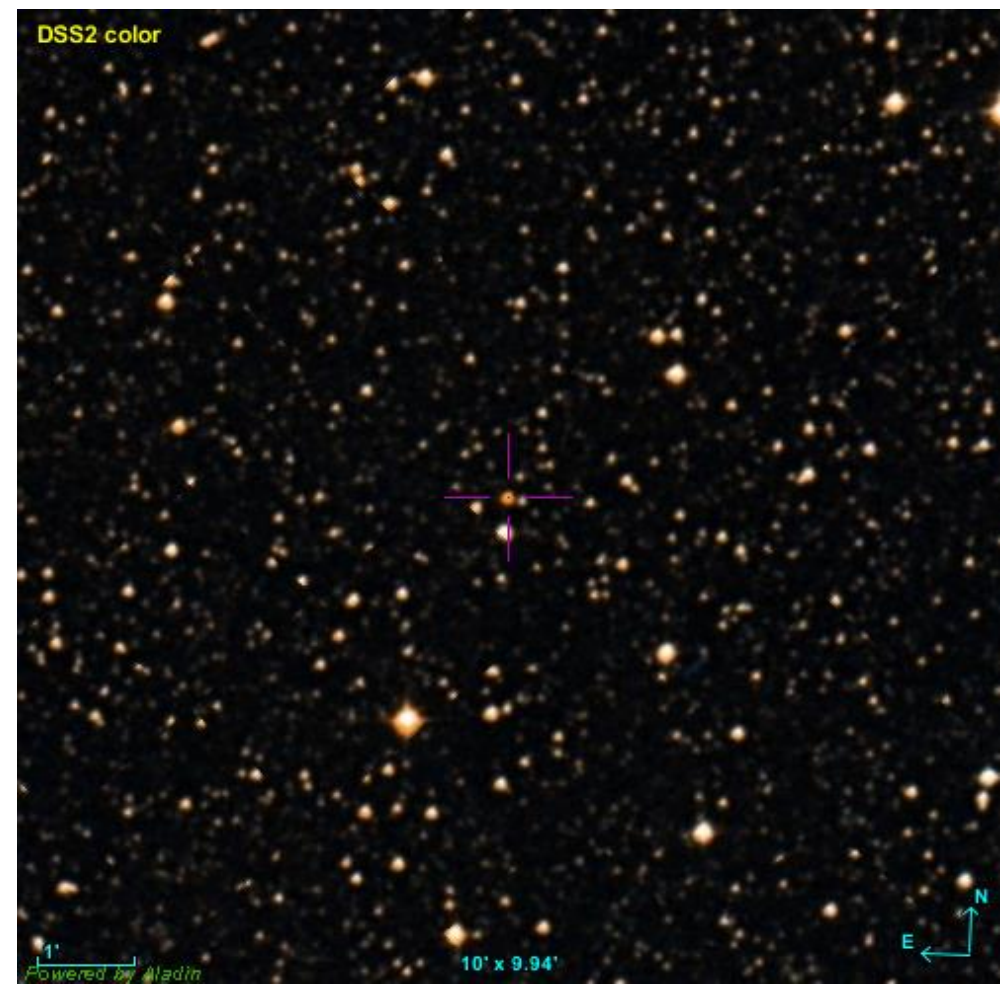

Figure 3.3 Sky region of ZTFJ173737.82-121047.1 (field of view 10' x 9.94').

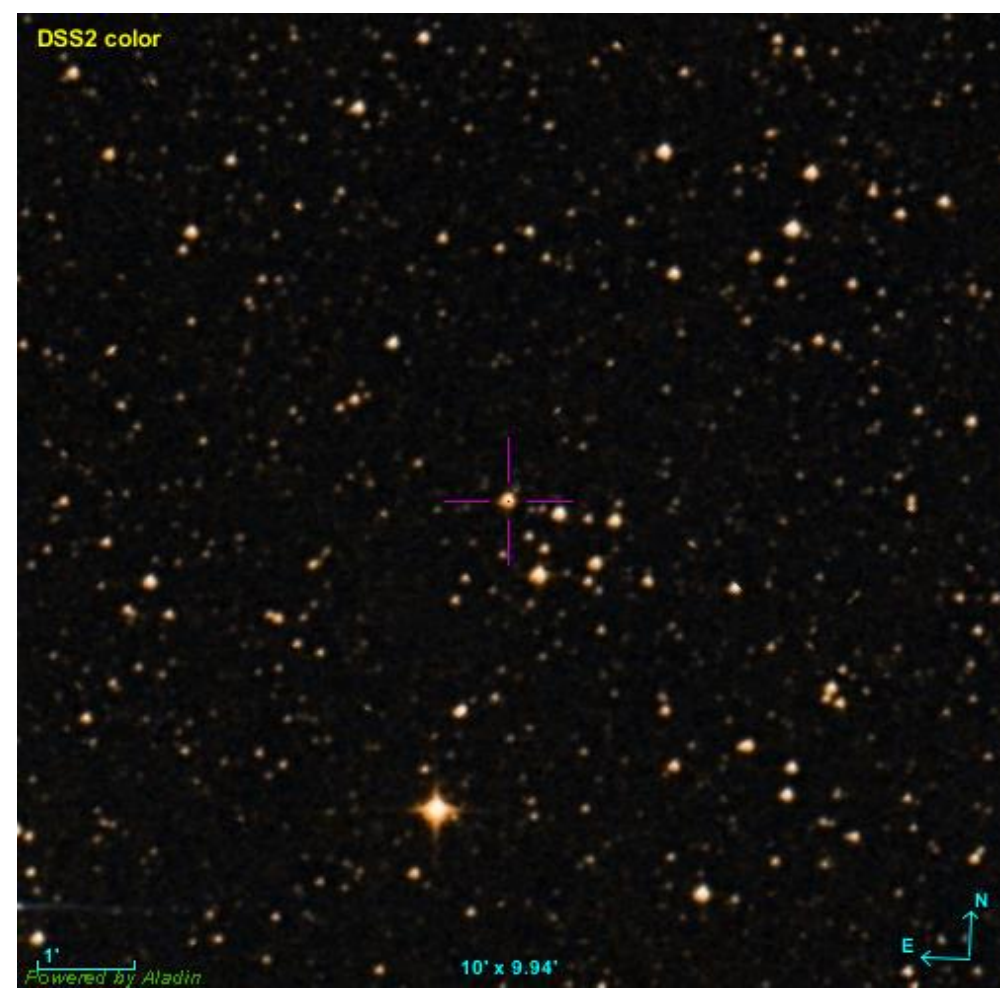

Figure 3.4 Sky region of ZTFJ174046.66-055611.9 (field of view 10' x 9.94'). 


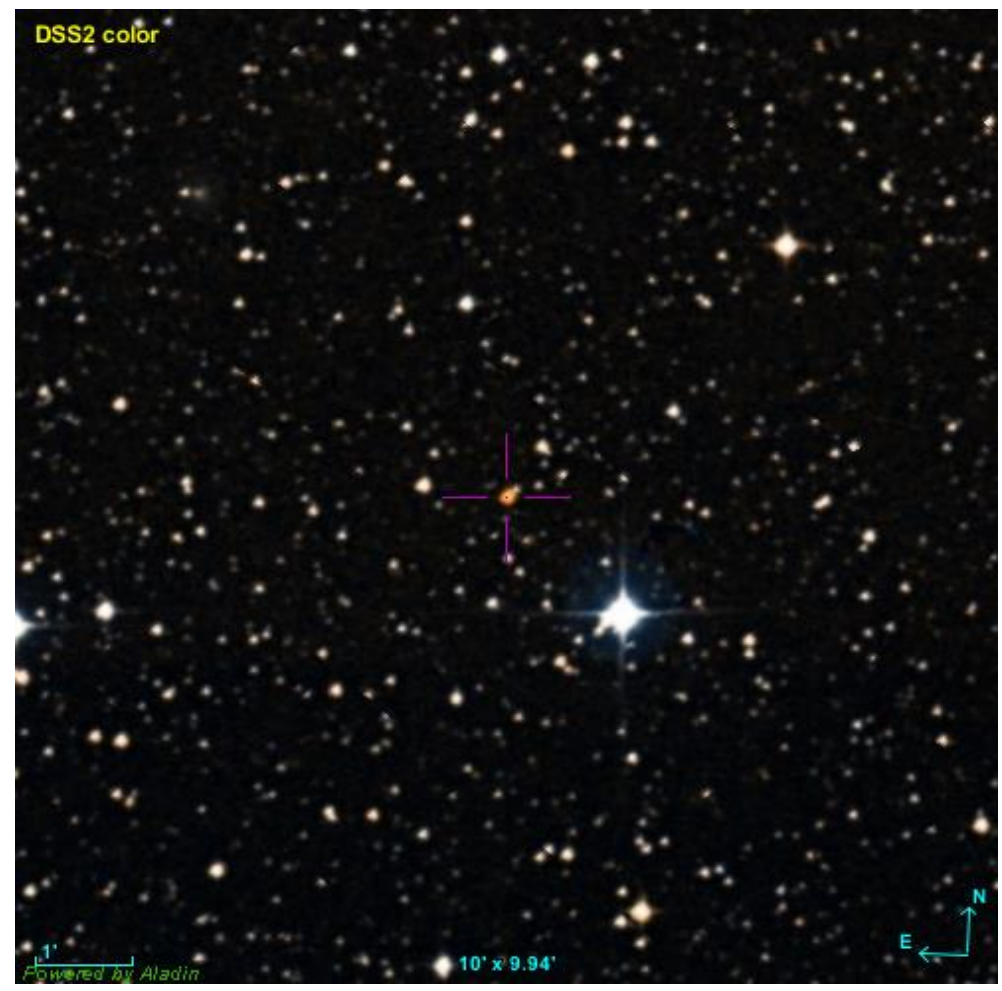

Figure 3.5 Sky region of ZTFJ174109.82-015331.2 (field of view 10' x 9.94').

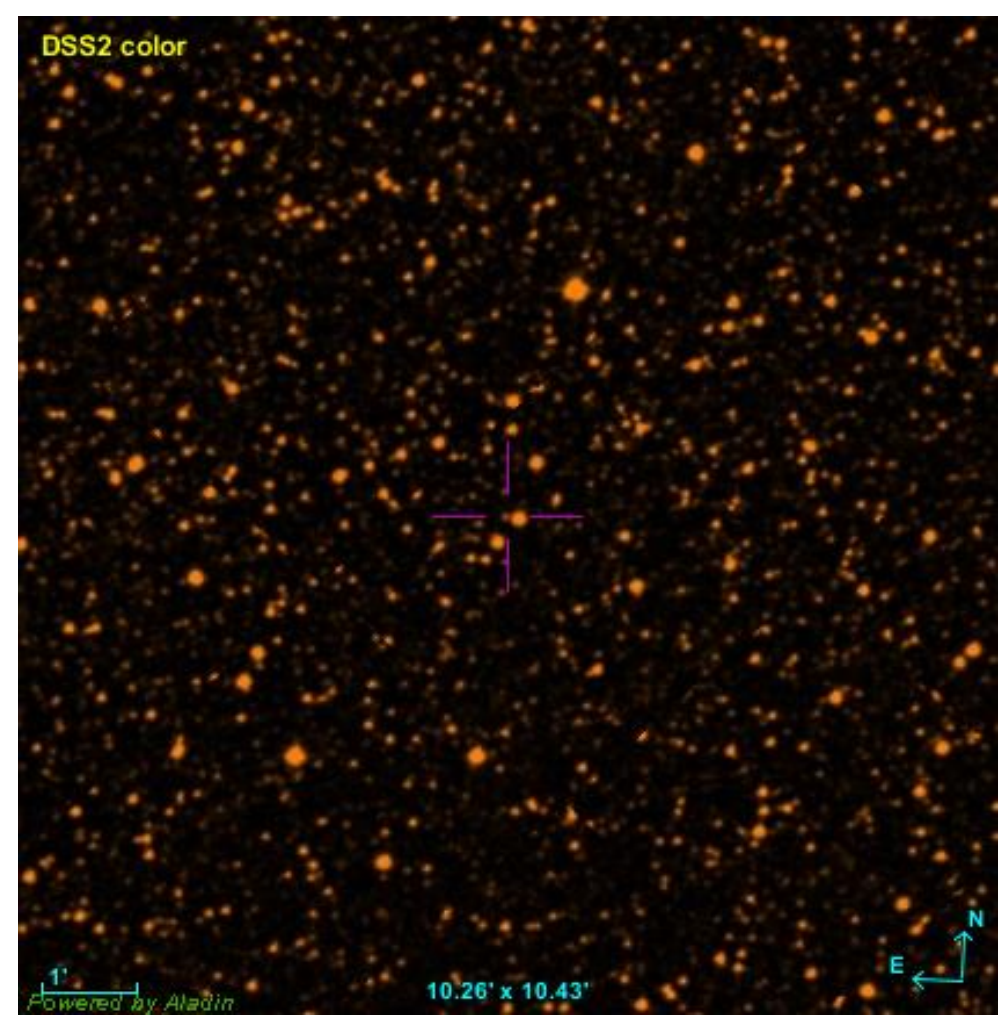

Figure 3.6 Sky region of ZTFJ174908.60-133931.5 (field of view 10.26' x 10.43'). 


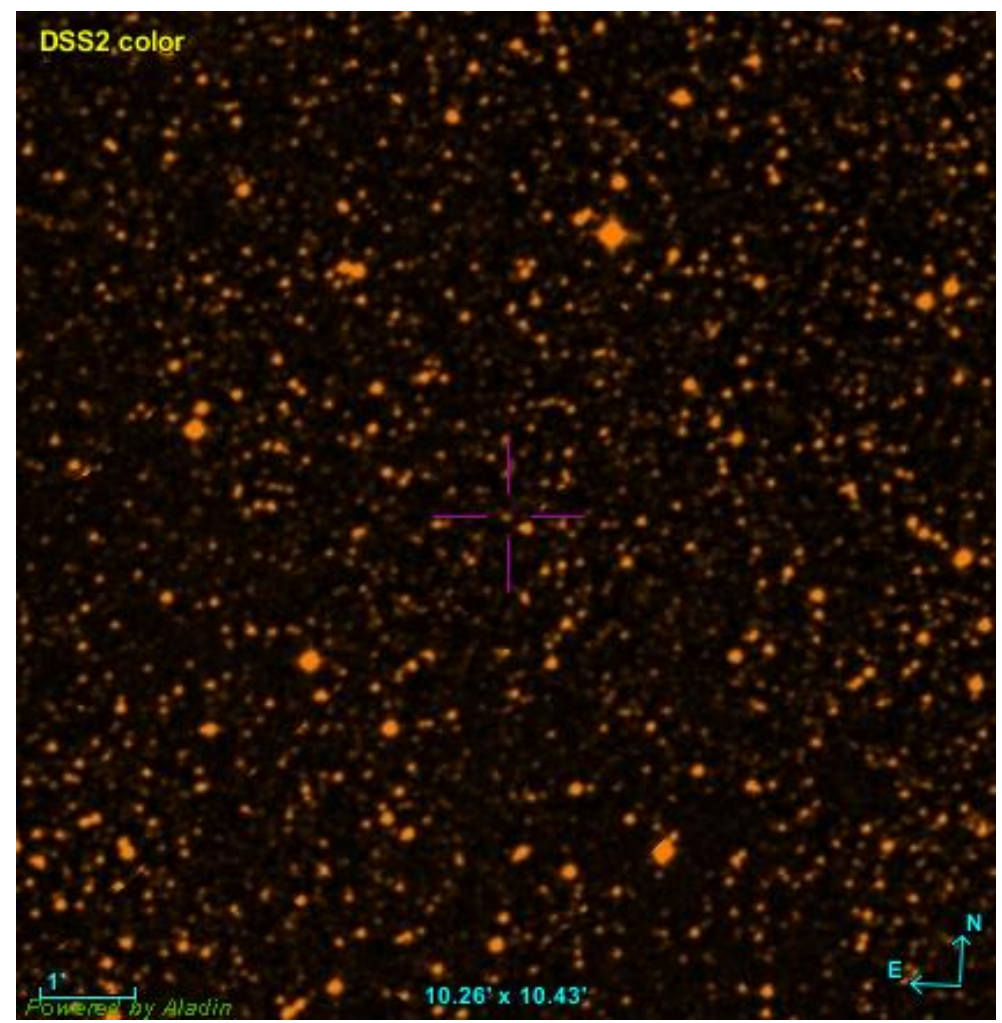

Figure 3.7 Sky region of ZTFJ174935.11-142533.9 (field of view 10.26' x 10.43').

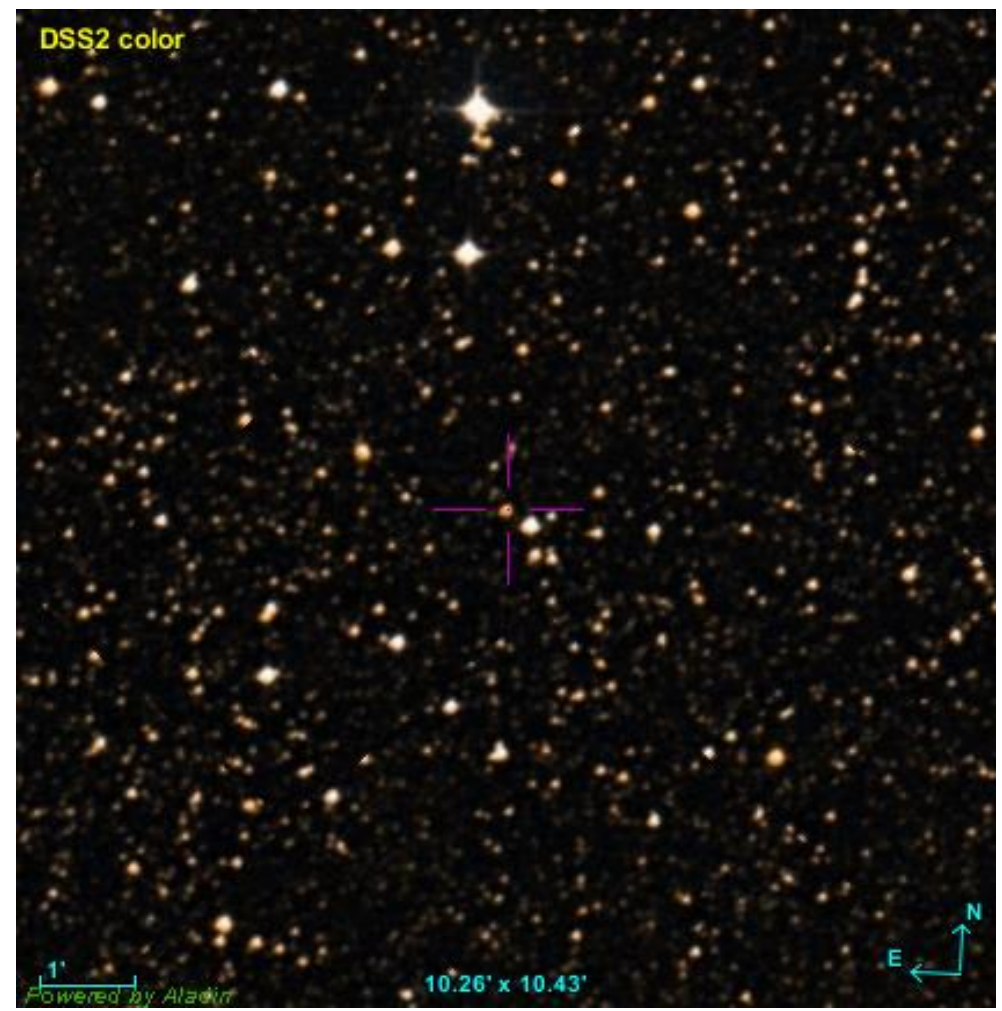

Figure 3.8 Sky region of ZTFJ175156.13-112355.2 (field of view 10.26' x 10.43'). 


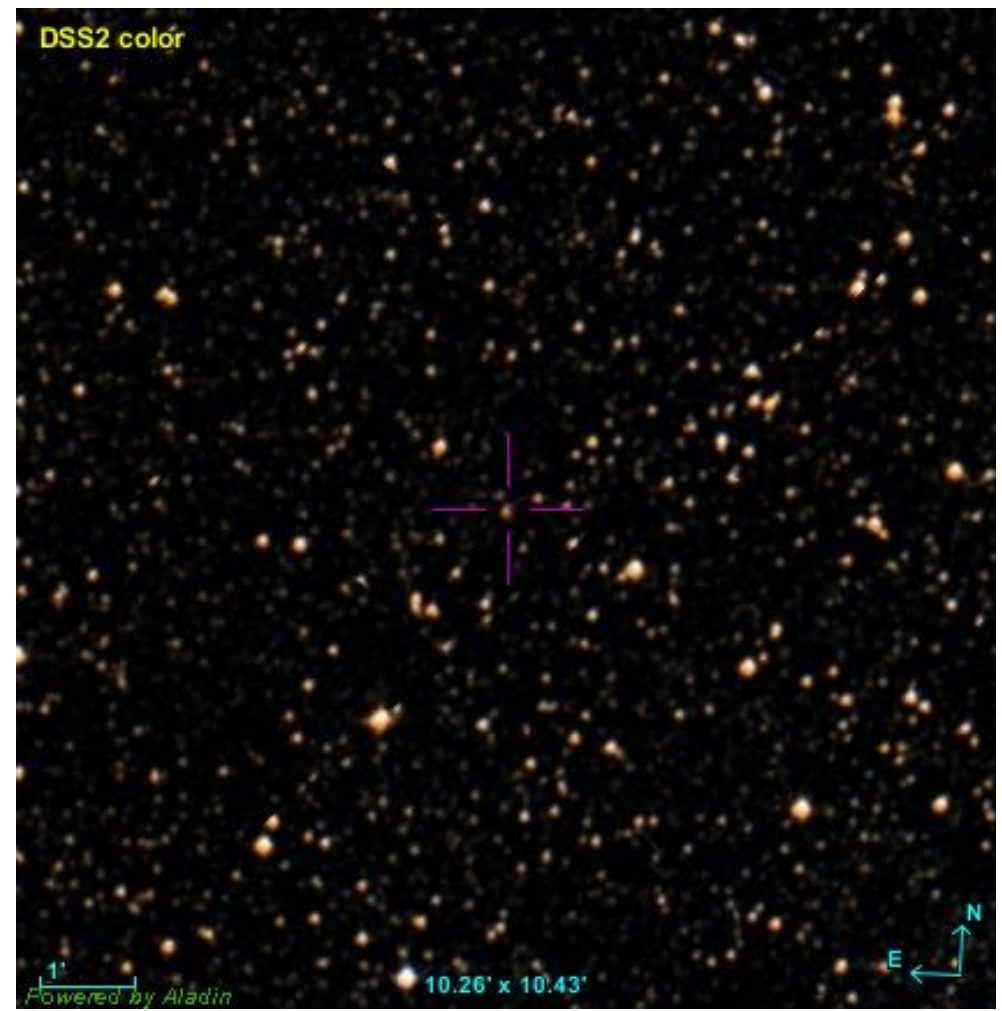

Figure 3.9 Sky region of ZTFJ175202.89-120213.0 (field of view 10.26' x 10.43').

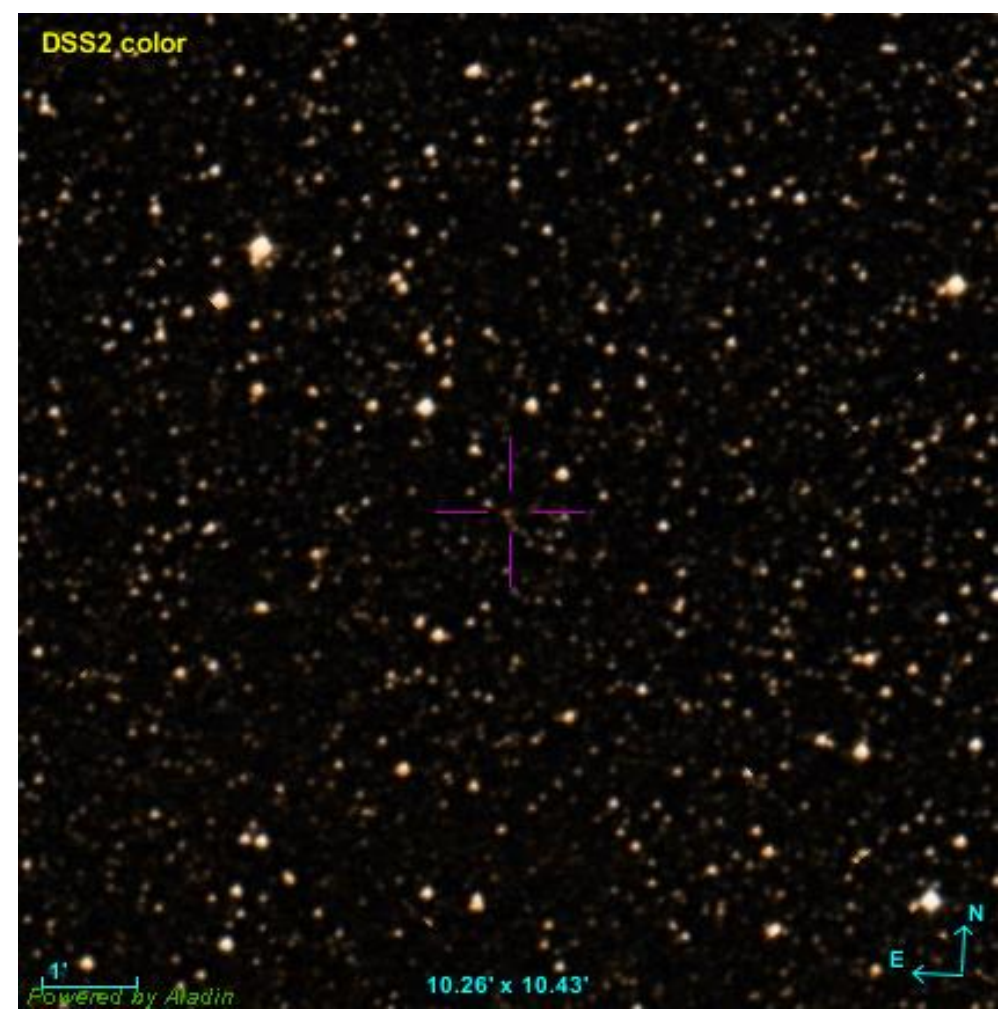

Figure 3.10 Sky region of ZTFJ175416.17-090917.0 (field of view 10.26' x 10.43'). 


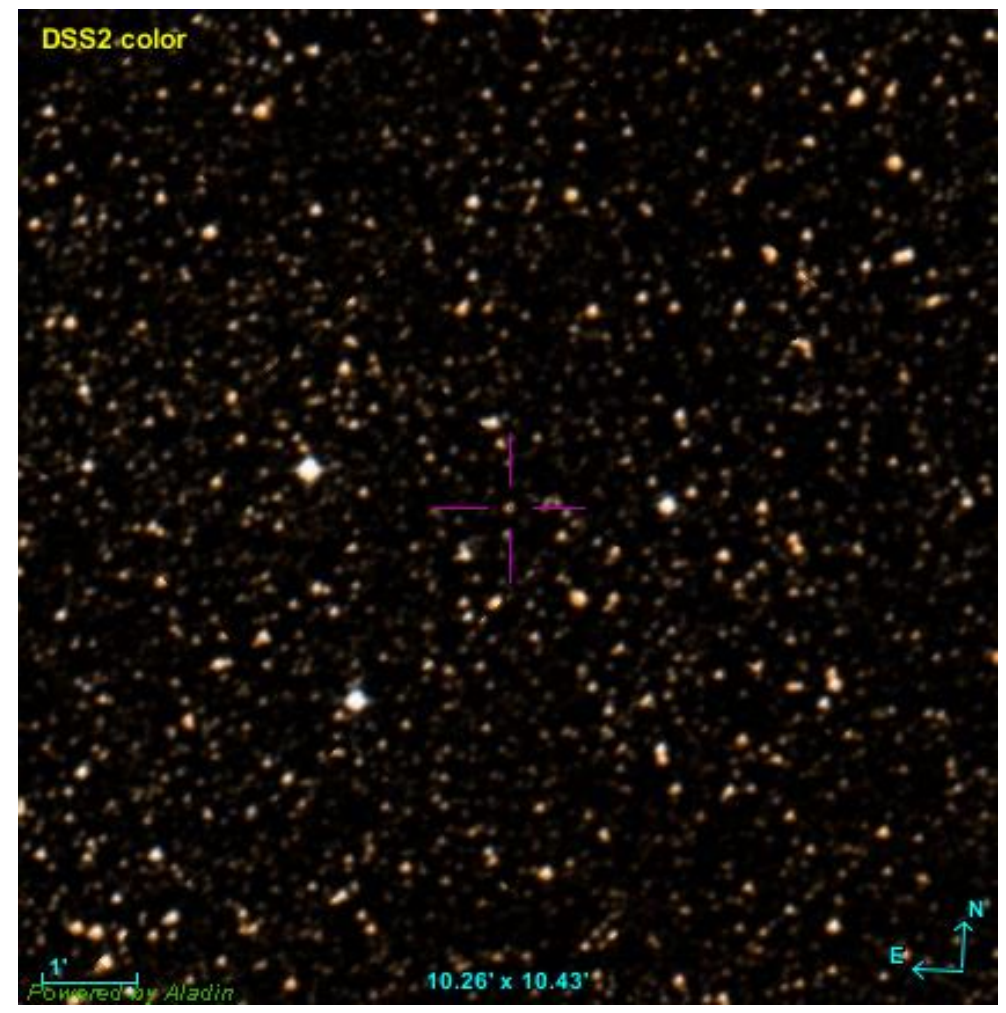

Figure 3.11 Sky region of ZTFJ175457.68-123542.7 (field of view 10.26' x 10.43').

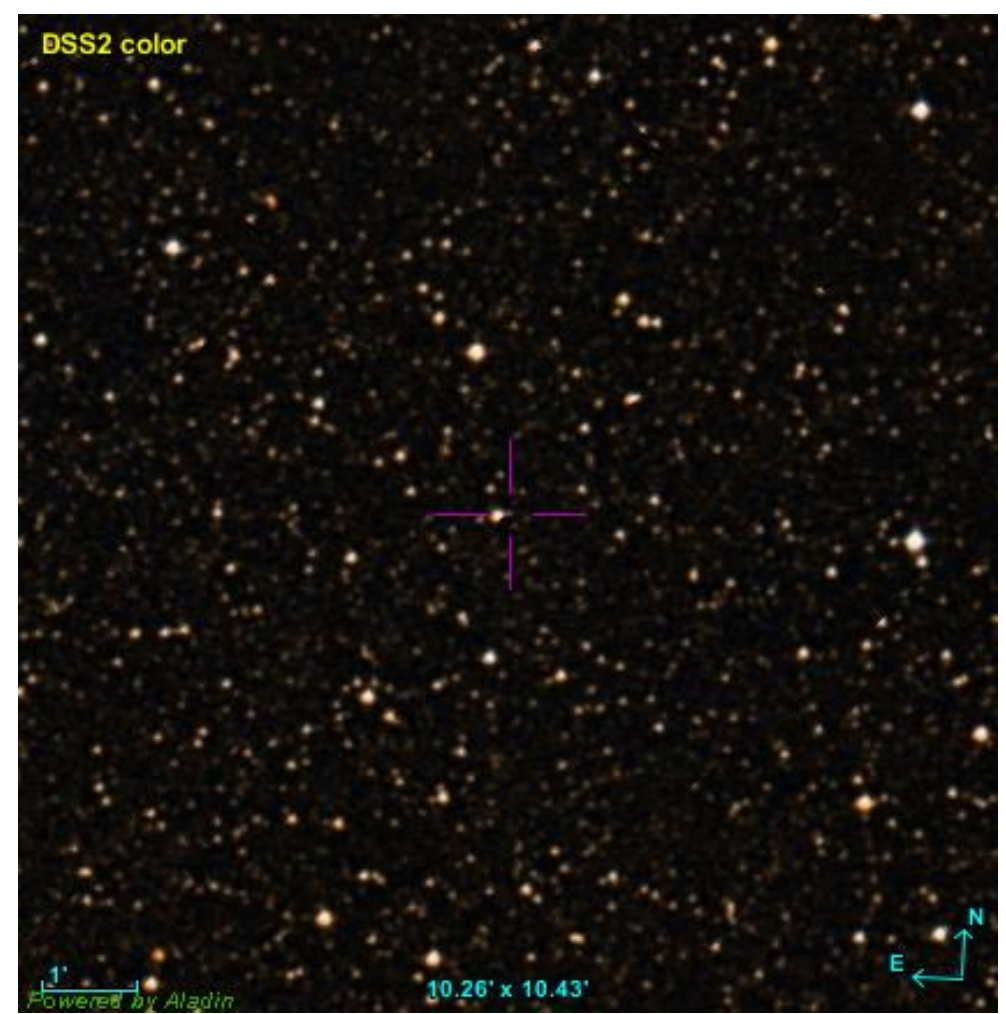

Figure 3.12 Sky region of ZTFJ175527.30-103939.8 (field of view 10.26' x 10.43'). 


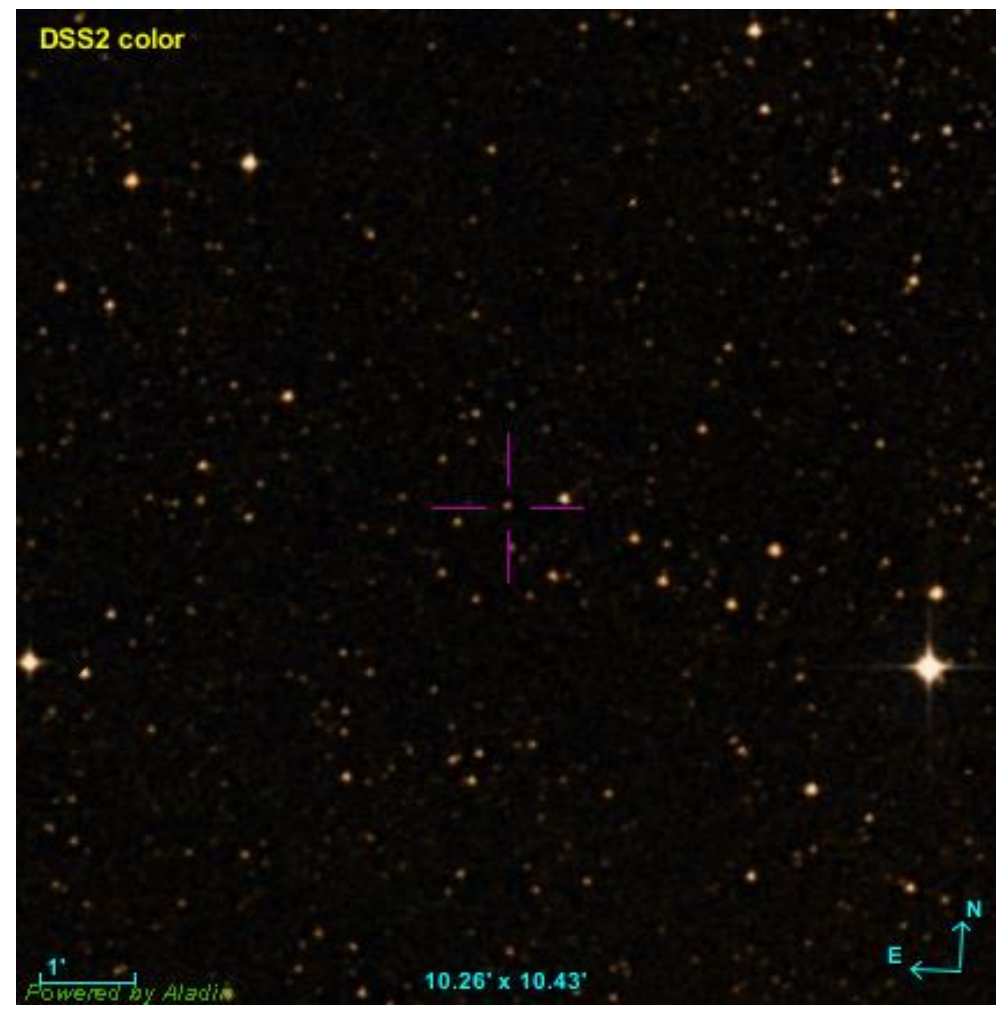

Figure 3.13 Sky region of ZTFJ175924.69-052150.2 (field of view 10.26' x 10.43').

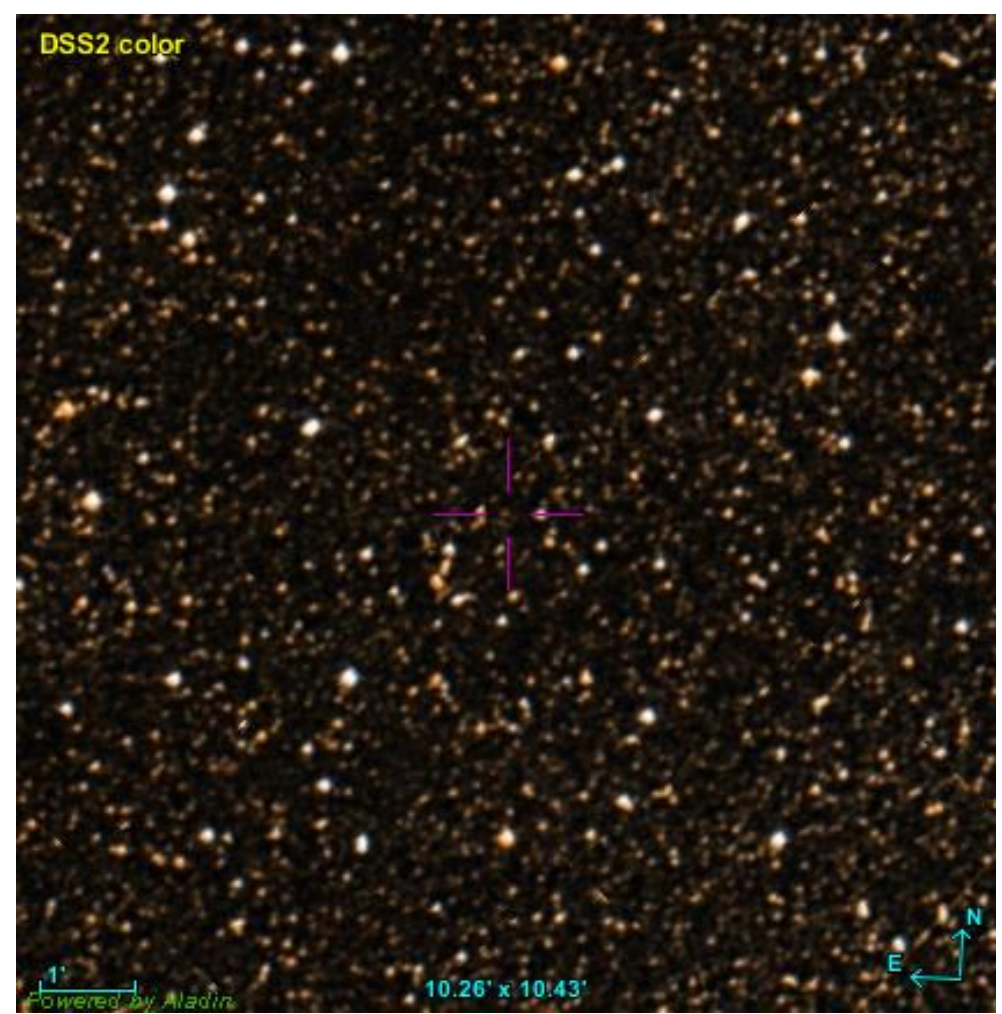

Figure 3.14 Sky region of ZTFJ180312.70-144808.1 (field of view 10.26' x 10.43'). 


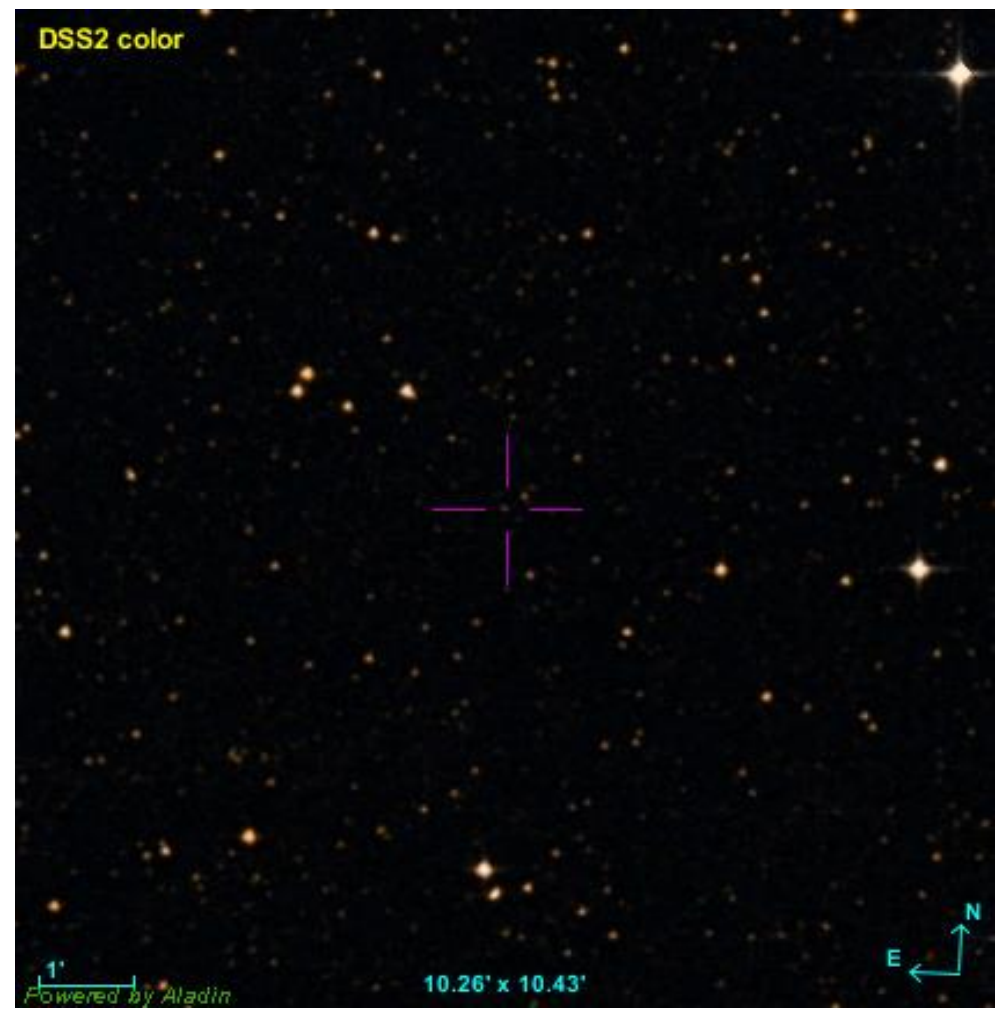

Figure 3.15 Sky region of ZTFJ180329.13-040204.5 (field of view 10.26' x 10.43').

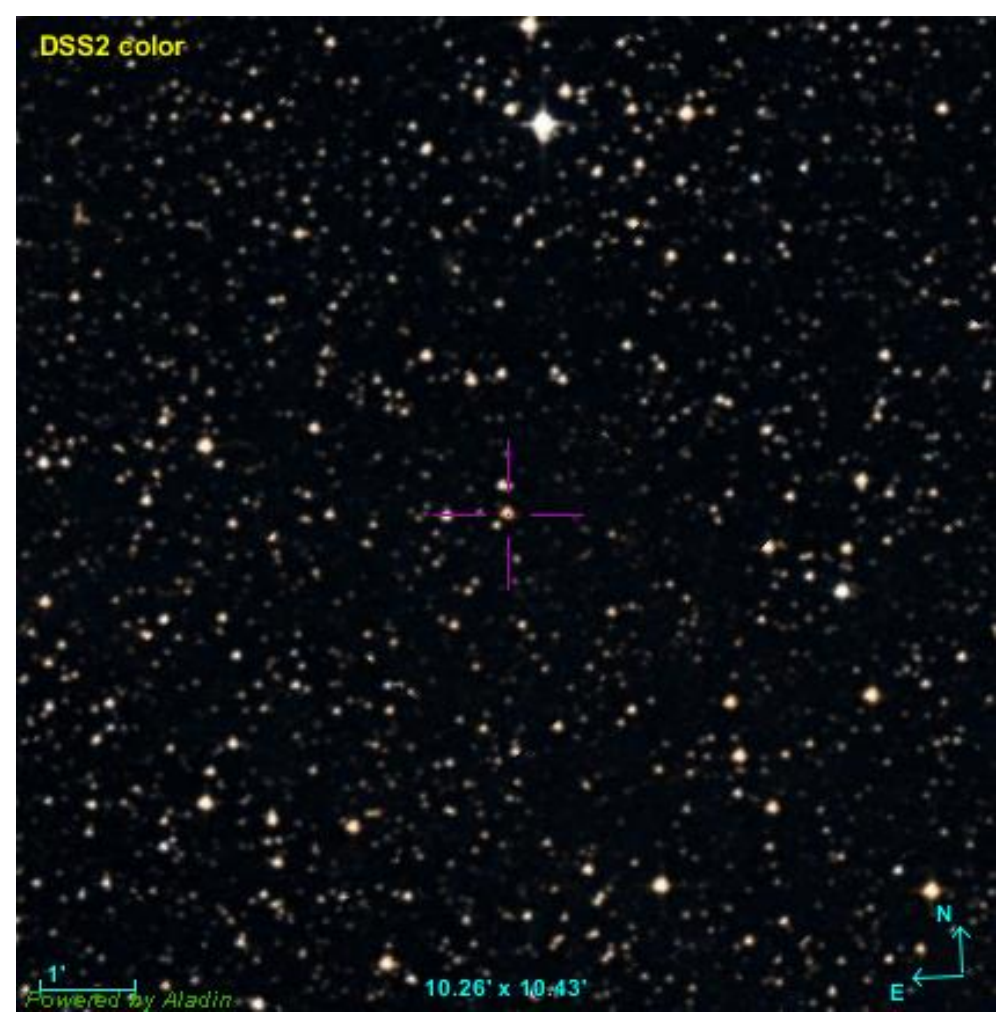

Figure 3.16 Sky region of ZTFJ180341.17+003614.0 (field of view 10.26' x 10.43'). 


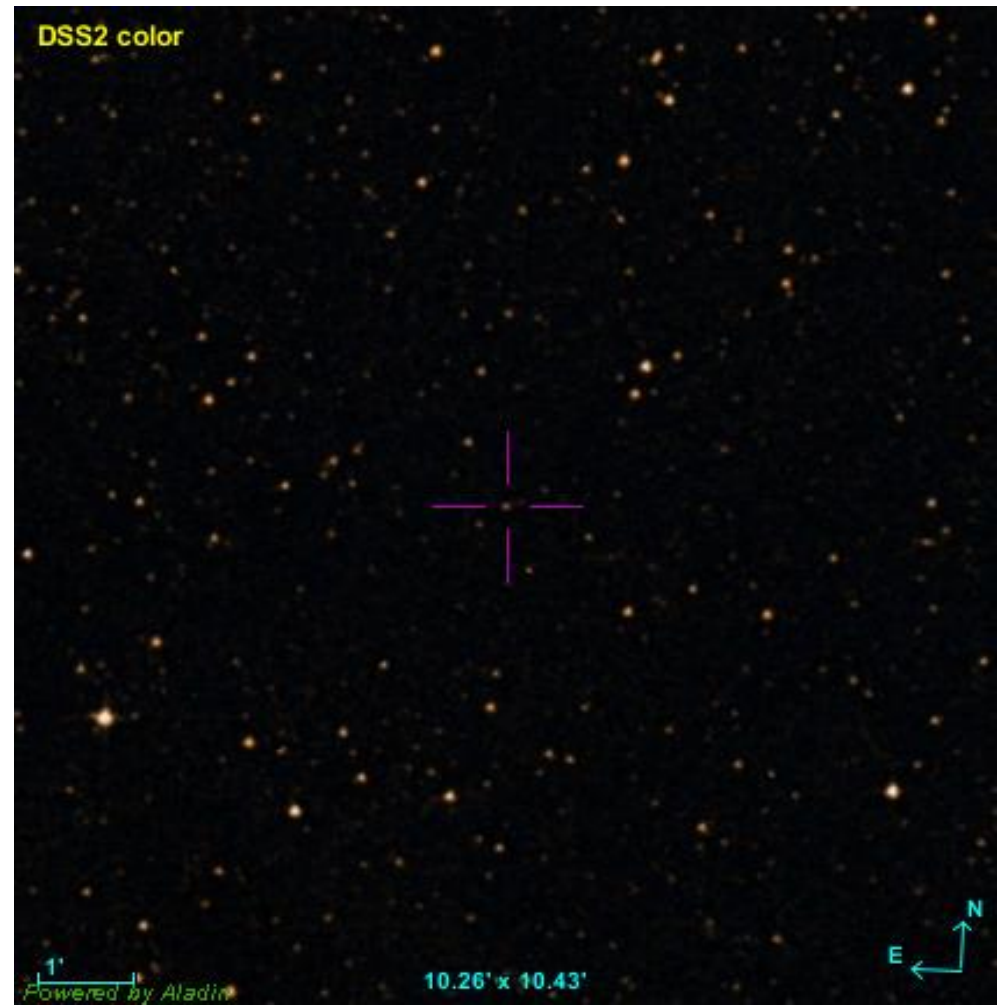

Figure 3.17 Sky region of ZTFJ180341.59-031849.0 (field of view 10.26' x 10.43').

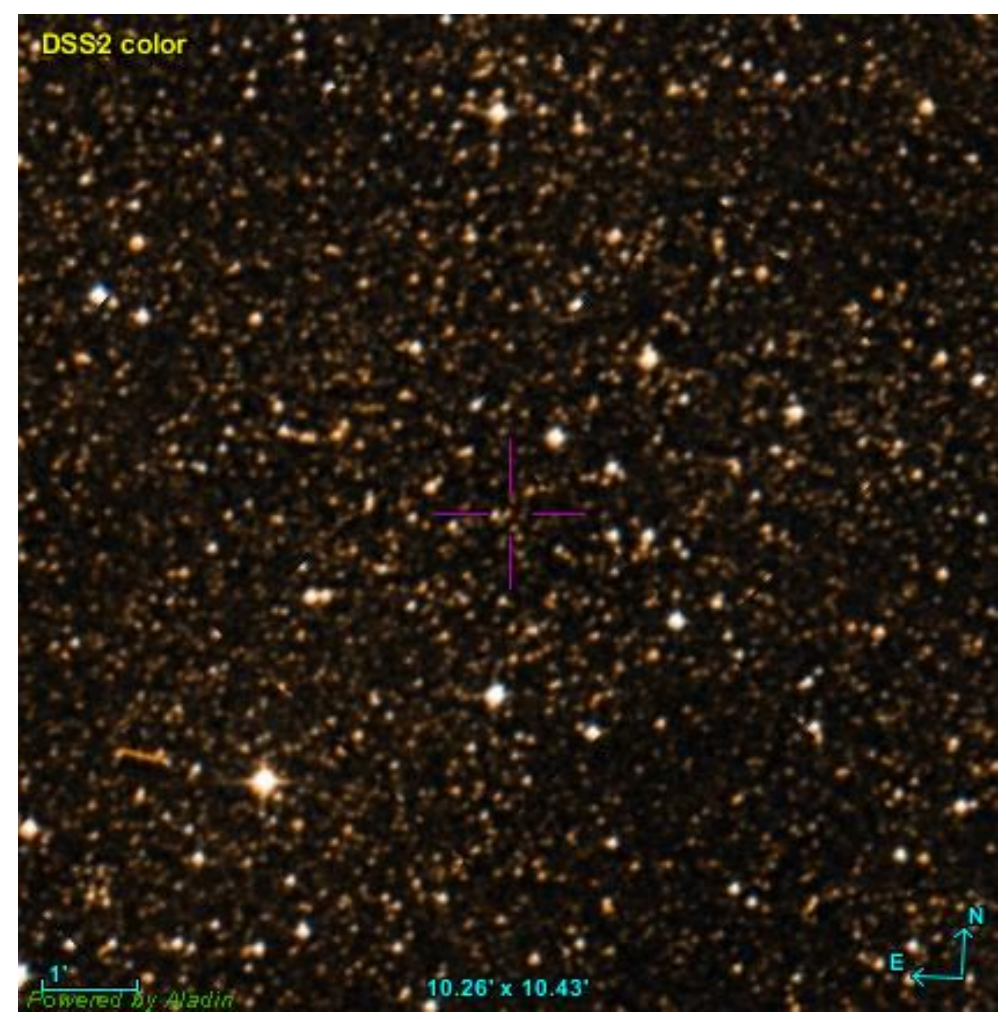

Figure 3.18 Sky region of ZTFJ180638.91-163509.8 (field of view 10.26' x 10.43'). 


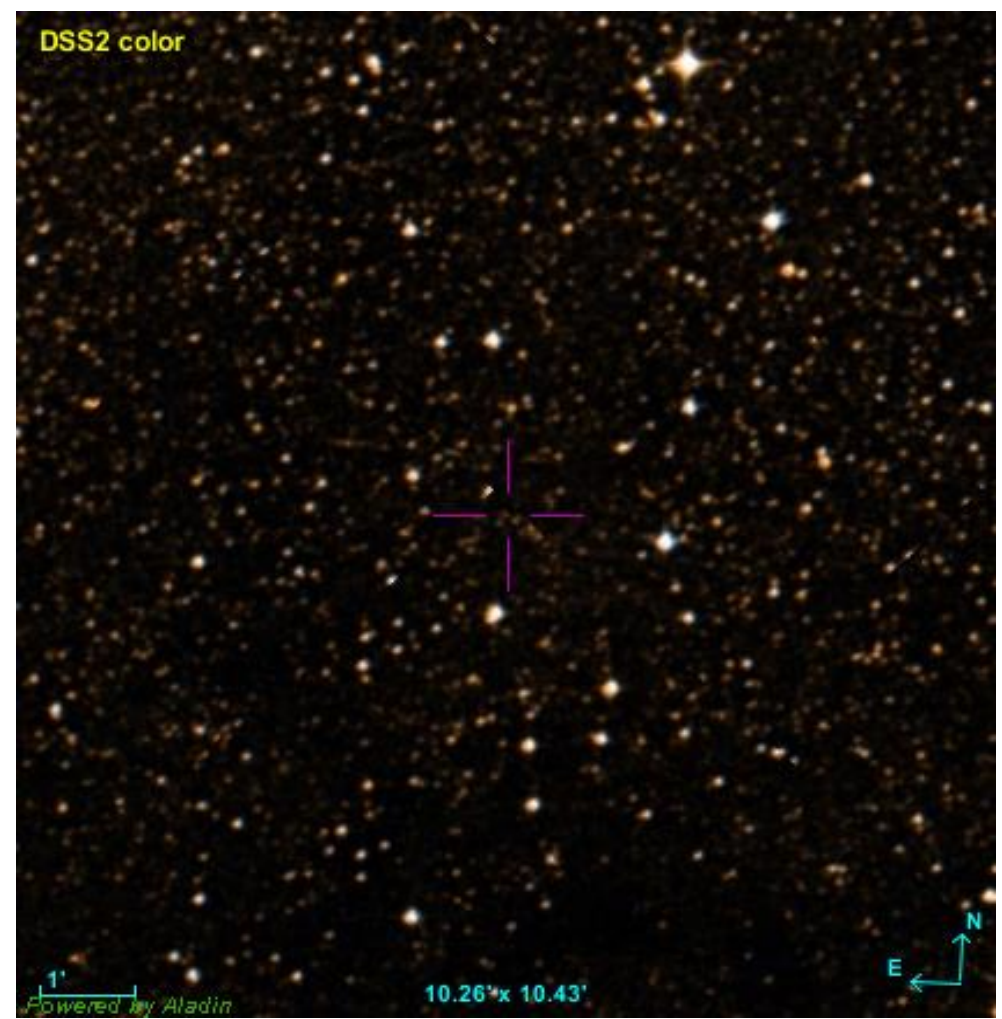

Figure 3.19 Sky region of ZTFJ180640.24-135159.8 (field of view 10.26' x 10.43').

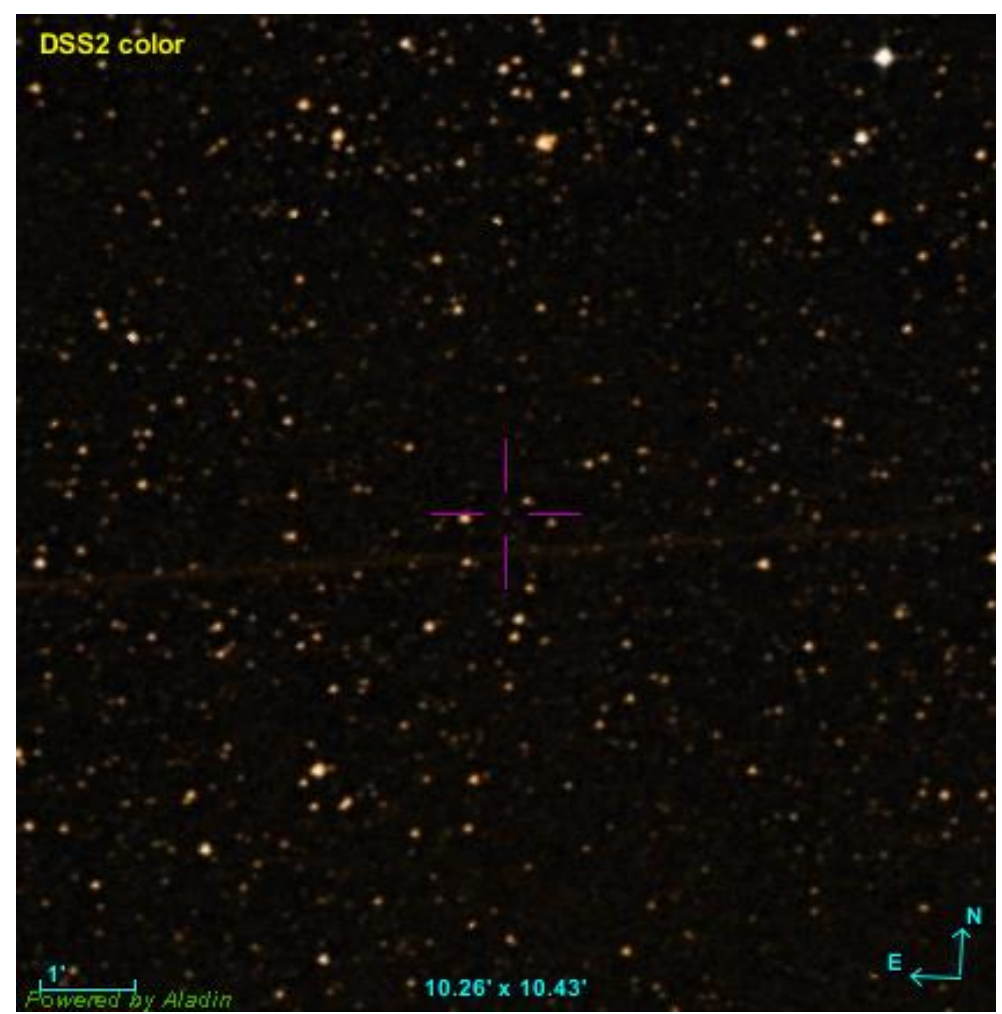

Figure 3.20 Sky region of ZTFJ180646.55-013545.0 (field of view 10.26' x 10.43'). 


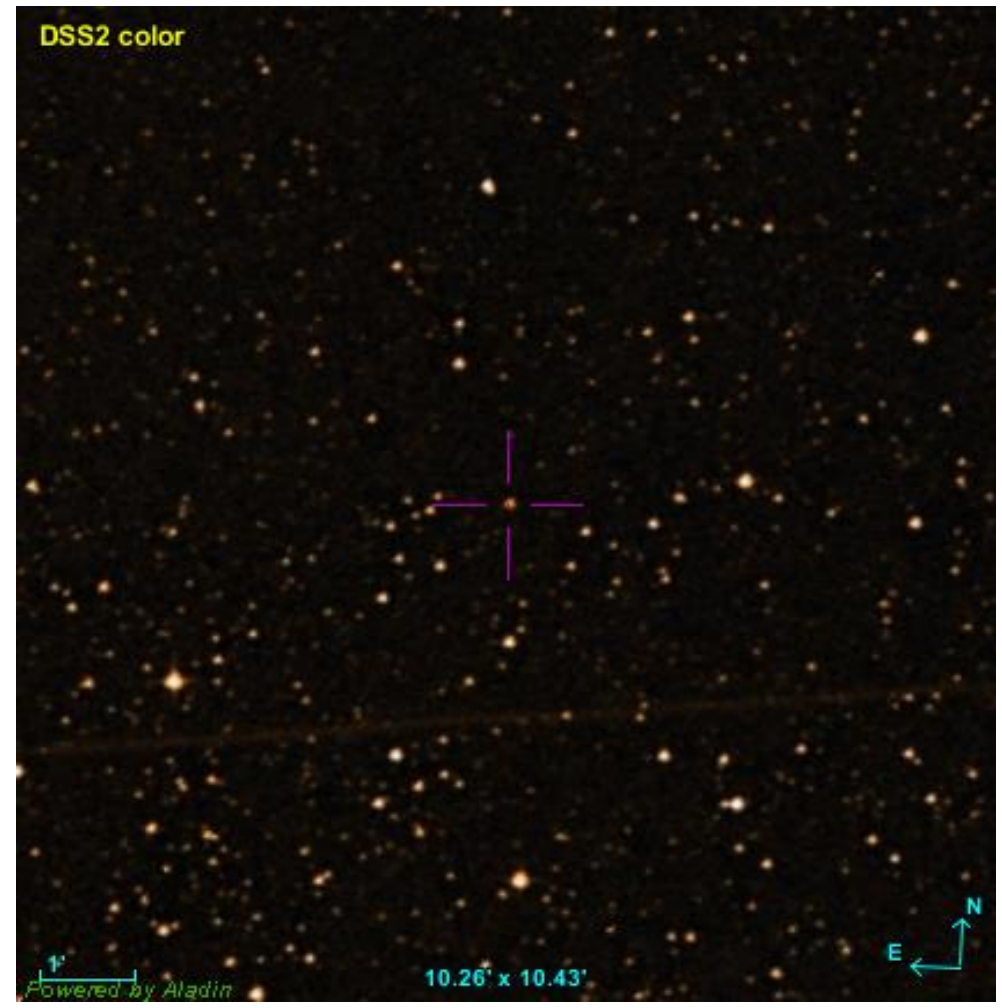

Figure 3.21 Sky region of ZTFJ180729.85-013438.1 (field of view 10.26' x 10.43').

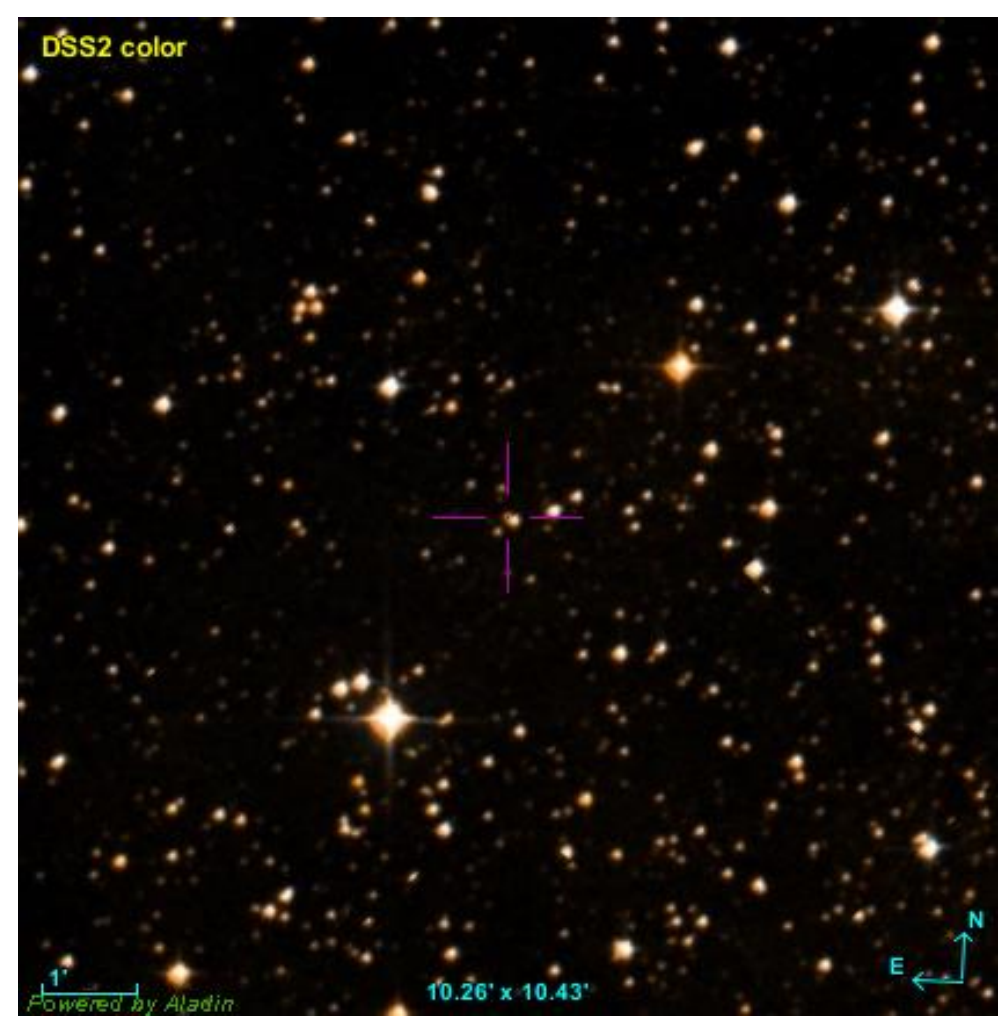

Figure 3.22 Sky region of ZTFJ180826.00-174129.0 (field of view 10.26' x 10.43'). 


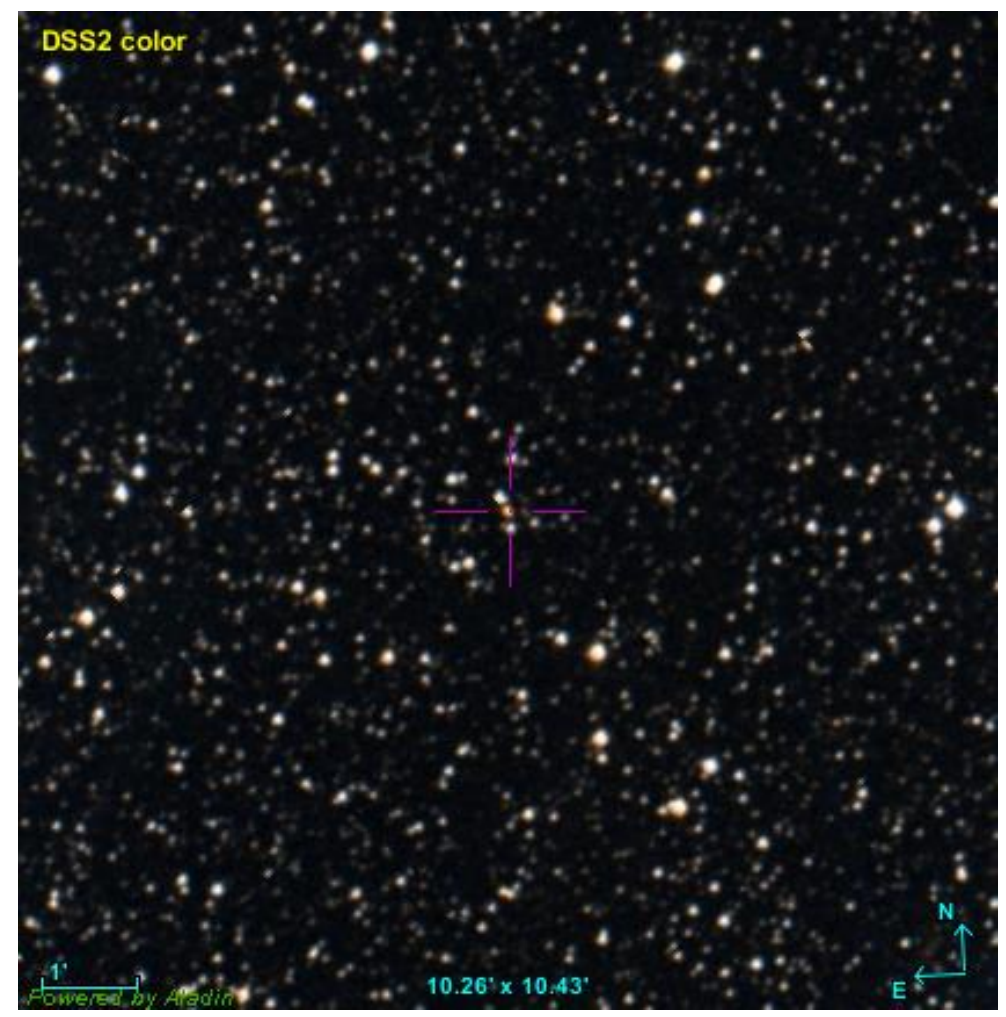

Figure 3.23 Sky region of ZTFJ184503.44+154335.3 (field of view 10.26' x 10.43').

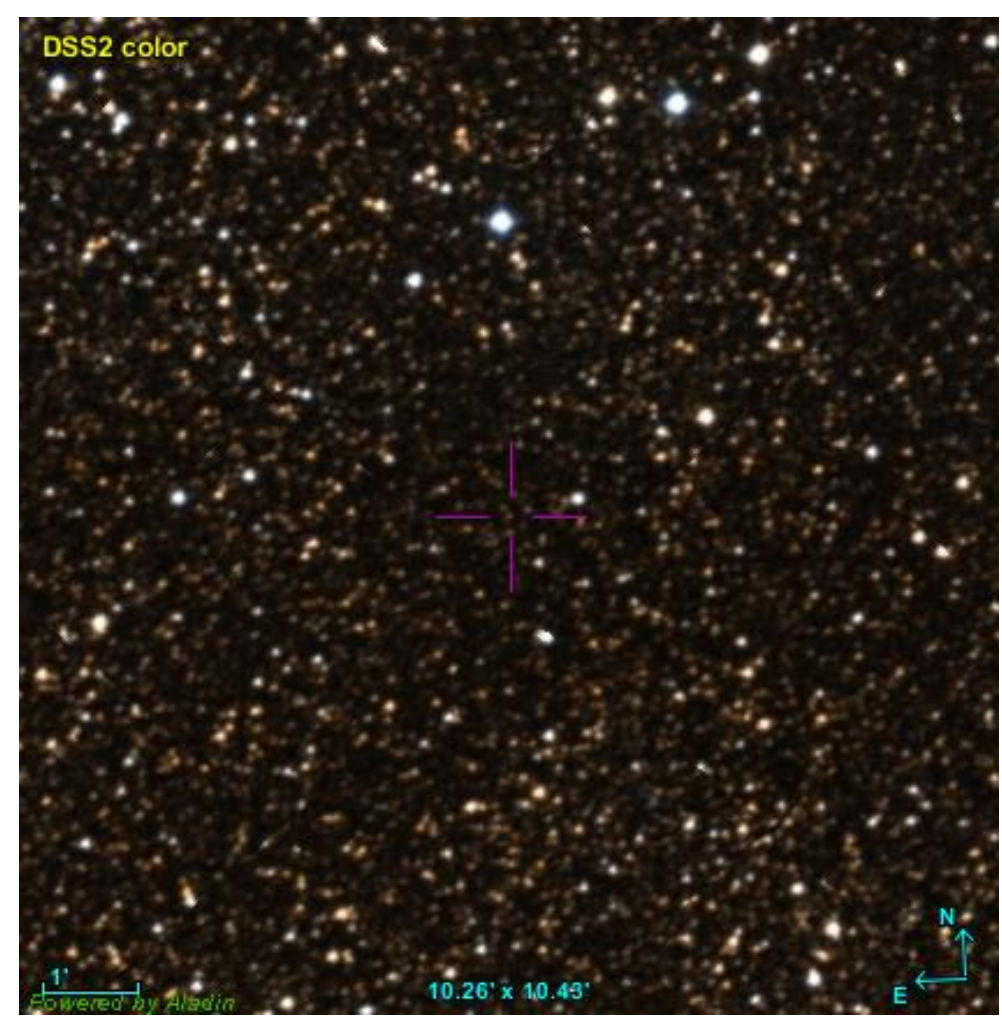

Figure 3.24 Sky region of ZTFJ190648.43+130203.5 (field of view 10.26' x 10.43'). 


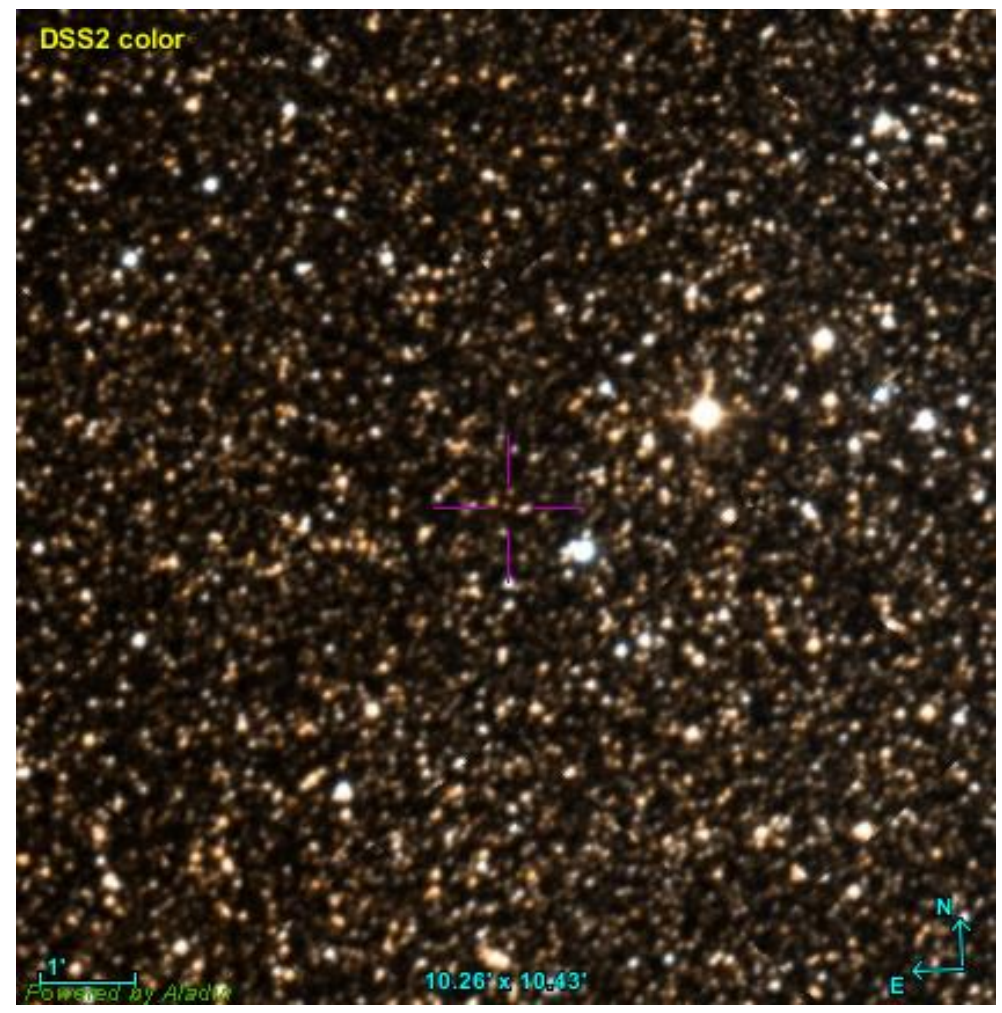

Figure 3.25 Sky region of ZTFJ191008.27+131014.4 (field of view 10.26' x 10.43').

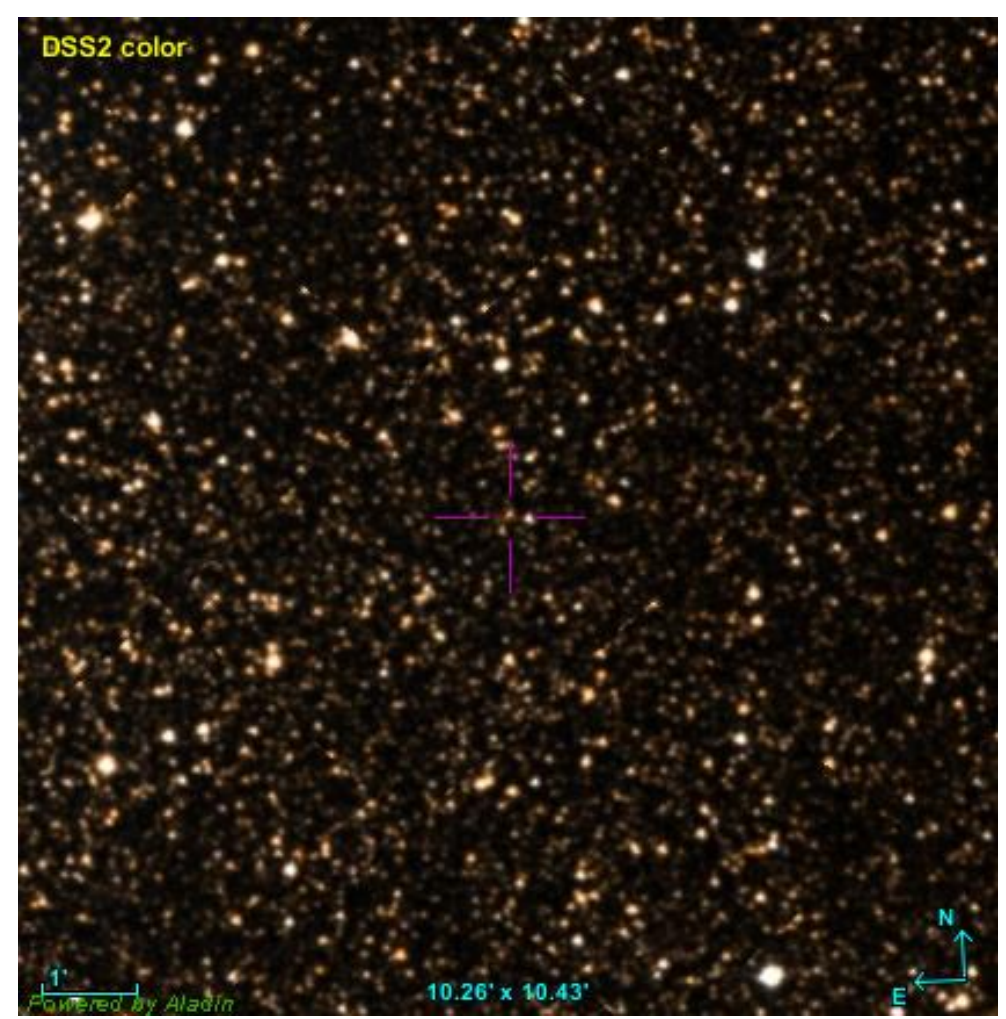

Figure 3.26 Sky region of ZTFJ191406.43+151810.6 (field of view 10.26' x 10.43'). 